\title{
A provenly correct translation of Fickle into Java ${ }^{\star}$
}

\author{
D. Ancona ${ }^{1}$, C. Anderson ${ }^{2}$, F. Damiani ${ }^{3}$, \\ S. Drossopoulou ${ }^{2}$, P. Giannini ${ }^{4}$, and E. Zucca ${ }^{1}$ \\ 1 DISI - Università di Genova \\ ${ }^{2}$ Imperial College - London \\ 3 Dipartimento di Informatica - Università di Torino \\ ${ }^{4}$ DISTA - Università del Piemonte Orientale
}

\begin{abstract}
We present a translation from $\mathcal{F}$ ickle, a small object-oriented language allowing objects to change their class at run-time, into Java. The translation is provenly correct, in the sense that we have shown it to preserve the static and dynamic semantics. Moreover, it is compatible with separate compilation, since the translation of a $\mathcal{F}$ ickle class does not depend on the implementation of used classes. Based on the formal system, we have developed an implementation.

The translation turned out to be a more subtle problem than we expected, and the proofs helped us uncover several subtle errors. In this paper, we discuss four different possible approaches we considered for the design of the translation and justify our choice, we present formally the translation and the proof of preservation of the static and dynamic semantics, and we discuss the prototype implementation.

The language $\mathcal{F}$ ickle has undergone, and is still undergoing several phases of development. In this paper we are discussing the translation of $\mathcal{F i c k l e}_{\mathrm{II}}$.
\end{abstract}

\section{Introduction}

Dynamic object re-classification is a programming language feature which allows an object to change its class membership at run-time while retaining its identity. Thus, one can express fundamental change of an object's behavior (e.g., nonempty lists becoming empty, iconified windows getting expanded, etc.) through re-classification, rather than through replacing objects of the old class by objects of the new class. Lack of re-classification primitives has long been recognized as a practical limitation of object-oriented programming. Fickle $[10-12,7-9]$ is a small Java-like language which supports dynamic object re-classification, aiming to demonstrate how object re-classification could extend an imperative, typed, class-based, object-oriented language.

\footnotetext{
* Partially supported by Dynamic Assembly, Reconfiguration and Type-checking EC project IST-2001-33477, APPSEM II - Thematic network IST-2001-38957, and Murst NAPOLI - Network Aware Programming: Oggetti, Linguaggi, Implementazioni.
} 
Several other approaches to the expression of fundamental change of behaviour have been suggested, see $[6,13,17]$. However, we claim that Fickle is more in line with the mainstream object oriented approach, whereby an object's behaviour is determined by its class membership. Moreover, $\mathcal{F}$ ickle is type-safe, i.e., any type correct program (in terms of the $\mathcal{F} i c k l e$ type system) is guaranteed never to attempt to access non-existing fields or methods.

Indeed, Fickle stands for a whole family of languages, which differ in the extent of the use of re-classifyable types, in the treatment of variables, in the support for class loading and the presence of threads. All $\mathcal{F}$ ickle languages support object re-classification, and are expression-oriented ${ }^{1}$.

Fickle, see $[10,11]$ is a Java-like language with a re-classification operation that changes the class membership of an object while preserving its identity. The basic problem in the design of the language is to have a sound type system, that is, a type system that, even in the presence of object re-classification insures that no attempt is made at accessing non existing members. This is obtained by tracking the type of the variables possibly denoting a re-classified objects. An object $\mathrm{o}$ of class c could also be denoted by a field $\mathrm{f}$ of type $\mathrm{d}$ (of some other object $o^{\prime}$ ), where $d$ is a superclass of c. Therefore, to achieve type soundness, we must forbid re-classification from (a subclass of) $d$ to a class which is not a subclass of d (otherwise subsequent accesses to members of o'.f existing in class d could fail). Classes for which this does not happen are said to be "respected" by re-classification and can be safely used as types for fields. In $\mathcal{F}$ ickle re-classificable classes are explicitly marked as state classes. State classes are the possible sources and targets of re-classifications, and root classes are the superclasses of state classes and declare all the members common to them. Only classes that are not subclasses of root classes can be used as types for fields (this classes are always respected by re-classification). Effects are sets of root classes, $\left\{c_{1}, \ldots, c_{n}\right\}$, meaning that there could be a re-classification between two subclasses of a class in the set. Re-classification is traced by effects, and methods are annotated with the effects that may be caused by the execution of their body. In Ficklethe mutation operation is only possible on the metavariable this, and in typing an expression we keep track of the current class of the metavariable this and the effects that are caused by the execution of the expression.

In $\mathcal{F}_{i c k l e}$ II [12] re-classification is possible on all the variables (not only on this as before). So in the type system we have to keep track of the current class of all the variables in the environment.

In $\mathcal{F i c k l e}_{\text {III }}$ [7] we refined the effect system with effects that are sets of pairs. The meaning of $\left\{\left(c_{1}, c_{1}^{\prime}\right), \ldots,\left(c_{n}, c_{n}^{\prime}\right)\right\}$ is that there could be a re-classification from $c_{i}$ to $c_{i}^{\prime}$. Fickle-style effect annotations can be coded into $\mathcal{F}_{i c k l e}{ }_{I I I}$-style effect annotations, but not vice versa, and $\mathcal{F}_{i c k l e} I I I$ allows to correctly type meaningful programs which $\mathcal{F}$ ickle rejects.

The $\mathcal{F}$ ickle $I_{I I}$-style of effects is used in $\mathcal{F}_{i c k l e_{3}}[8]$, to eliminate the explicit marking of classes for which re-classification is allowed (state and root), since this

\footnotetext{
${ }^{1}$ We chose to express our ideas in an expression-oriented setting, as this greatly facilitated the formal treatment.
} 
information can be inferred from the effects. In this way we obtain a language that is syntactically simpler, and allows unanticipated re-classification. However, this has a drawback: when linking a class $c$ with an existing set of classes we have to check on one side that the effects of the methods of $c$ respect the classes used as types of fields of the preexisting classes, and on the other that the effects of the methods of the preexisting classes respect the classes used as types of fields of $c$.

Finally in $\mathcal{F i c k l e}_{M T}[9]$ we consider the issue of dealing with re-classification in the presence of multi-threading. To this aim we define a multi-threaded extension of the language $\mathcal{F} i c k l e$ (in which re-classification is only allowed on this), that we call $\mathcal{F}_{i c k l e}{ }_{M T}$. In $\mathcal{F}_{i c k l e}{ }_{M T}$ the expression spawn(e) starts the evaluation of the expression e in a new thread while the current thread continues by evaluating the expression following spawn(e). The new and the current thread work on a common heap containing the set of defined objects, and, through aliasing, re-classification may change the class membership of objects across threads. To achieve soundness we prevent executions in which a thread re-classifies an object while another is executing a method on the object. This is done by combining the static type and effect system (defined for $\mathcal{F}$ ickle) with a synchronization mechanism based on effect information that delays the execution of possibly dangerous threads. We can prove that, on one side no execution of a well-typed expression can cause the access to non-existing members of objects, and on the other the delays introduced do not cause deadlocks.

In this paper we investigate the practicality of the ideas around Fickle, through the discussion of an an implementation, which maps $\mathcal{F}$ ickle onto Java. From the languages in the $\mathcal{F}$ ickle family, we chose $\mathcal{F} i c k l e_{\text {II }}$, which in some sense is the "essence" of re-classification, as it allows parameters and return types of re-classifyable type, but does not support class loading or threads. We chose this version of $\mathcal{F}$ ickle, because it is the most expressive language from the $\mathcal{F}$ ickle family which is solely concerned with re-classification for object-oriented languages, ${ }^{2}$ and also because we have published a complete semantics and proofs of soundness for $\mathcal{F}_{\text {ickle }}$ II $[12]$.

We wanted to study the problem of simulating re-classification from its theoretical and its practical aspect. Therefore, we proceeded by developing in parallel both a formalization of the translation, and a prototype implementation. The translation turned out to be a more complex task than we had originally anticipated, and several subtle issues had to be considered. Thus, the formalization served to elucidate the design, and the properties of the formalization helped us in identifying and addressing the aforementioned subtle issues. The formalization maps $\mathcal{F}_{i c k l l_{\text {II }}}$ into $\mathcal{F} i c k l e_{\text {II }}^{-}$, where $\mathcal{F}$ ickle $e_{\text {II }}^{-}$is a fragment of $\mathcal{F} i c k l e_{\text {II }}$ without re-classification - we chose $\mathcal{F}$ ickle $e_{\text {II }}^{-}$because this allowed us to use the same type system and operational semantics as for $\mathcal{F}_{i c k l e}$ II. Our prototype implementation [5] maps $\mathcal{F} i c k l e^{\text {st }}$ onto Java. $\mathcal{F} i c k l e^{\text {st }}$ is a statement oriented version of $\mathcal{F} i c k l e_{\text {II }}$.

\footnotetext{
${ }^{2}$ Fickle $_{\text {II }}$ is most expressive with regards to re-classification, but does not have the extra features supporting unanticipated software evolution, nor has it got the multithreading support as in $\mathcal{F}_{\text {ickle }}{ }_{I I I}$ and $\mathcal{F}_{\text {ickle }}{ }^{m}$.
} 
The translation is provenly correct: We present here the proofs that it preserves the static and dynamic semantics - i.e., well-formed $\mathcal{F}_{i c k l}$ II programs are translated into well-formed $\mathcal{F} i c k l e_{\text {II }}^{-}$programs which behave "in the same way".

Moreover, the translation is compatible with separate compilation, in the sense that the translation of a $\mathcal{F} i c k l e_{\text {II }}$ class does not depend on the implementation of any classes it uses. Therefore, our translation could form the basis for an extension of a Java compiler; namely any type information needed by the translation can be retrieved from type information stored in binary files, as is done, indeed, by Java compilers for ordinary compilation.

For the design of the translation we had to consider the following issues:

1. an appropriate encoding for re-classifiable objects,

2. the relation between the types of a $\mathcal{F}_{i} c k l e_{\text {II }}$ expression and the corresponding translated Java expression,

3. ensuring that a translated Java expression of class type will always denote the object in its most current state,

4. the fact that a standard Java class $c$ can be extended by a re-classifiable class, possibly after $c$ has been translated (i.e., compiled),

5. making the translation compatible with separate compilation.

Concerning point 1), the basic idea is to represent each re-classifiable $\mathcal{F} i c k l l_{\mathrm{II}}$ object $o$ through a pair $\langle i d, i m p>$ of Java objects. Roughly speaking, $i d$ provides the (immutable) identity of $o$, whereas imp is the implementor object of the id object, and provides its (mutable) behavior. A re-classification of $o$ changes imp but not $i d$, and method invocations are resolved by $i m p$.

Concerning point 2), our initial idea was that there should be an isomorphism between the types of $\mathcal{F} i c k l e_{\text {II }}$ expressions and the types of the translated Java expressions. However, as we shall argue in section 7, this lead to a complex system, and made the issues around point 3 ) more difficult. In our current solution all $\mathcal{F}$ ickle II $_{\text {I }}$ expressions of class type are translated to Java expressions of the same type, namely Identity.

Concerning point 3 ), originally we were maintaining a chain of objects where each was delegating to the next, more recent implementation object. Thus, every field access or method call needed to follow the chain in order to find the most recent implementation object. However, this solution grew rather complex, and we abandoned it for the pair $\langle i d, i m p>$ described above.

Concerning points 4), and 5), we decided to represent all objects, even the non-re-classifiable ones, through such pairs $\langle i d, i m p\rangle$. Thus, all Fickle classes, even the ones that describe objects which may not be re-classified, are translated in a uniform way. Also, field accesses or method calls need to find the implementation object, and are therefore translated in a uniform way, independently of whether the receiver belongs to a re-classifyable class, or not. Thus, a class may be translated without internal knowledge of the classes it is using.

Lastly, we also had to reconcile the requirements for the production of efficient Java code, the simplicity of the translation, and simplicity of the proofs. We 
decided to make the translation as simple as possible, neglecting efficiency in favor of uniformity (i.e., fewer different cases) and simplicity.

The work presented in this paper comes out of the collaboration of three different research groups and is based on their previous experience in the design and implementation of Java extensions $[3,12]$. All sites have been involved in the design of the translation and its properties.

The formal work has been taking place in parallel with the development of prototype implementations, and there was considerable cross-fertilization. The first implementation, Carmela [4], together with an extensive test-suite, was developed largely before the formalization, but as it grew rather complex we introduced the concepts of the formalization to reason about it. We then uncovered some problems, and modified the approach [1,2]. The current paper is the culmination of our experience with various approaches, and presents the simplest design. The approach has been implemented in Isabella [5]. We have developed full proofs of correctness.

The paper is organized as follows: In Section 2 we introduce $\mathcal{F}$ ickle $e_{\text {II }}$ informally in terms of an example. In Section 3 we give a brief formal description of $\mathcal{F}_{i c k l e}$ II (syntax, operational semantics, and typing) and state the soundness of the type system. In Section 4 we give an informal overview of the translation, while in Section 5 we give the formal description. In Section 6 we state the properties of the translation (preservation of static and dynamic semantics) and illustrate the compatibility of the translation with Java separate compilation. In Section 7 we discuss the various design alternatives considered for the translation. In Section 8 we describe our current implementation of the translation [5]. We conclude by summarizing the relevance of this work and discussing further research directions. Some technical definitions are illustrated in Appendix A. Proofs of the main results are given in Appendices B and C.

\section{Fickle $_{\text {II }}$, an informal overview}

In this section we introduce $\mathcal{F}$ ickle $e_{\text {II }}$ informally using an example.

The $\mathcal{F}_{i c k l e}$ II example in Fig. 1 describes accounts which belong to people, and which may be daily accounts, or savings accounts. It consists of a class Person, and a class Account, with subclasses SavingsAccount and DailyAccount. An Account belongs to a person (field Person owner), and holds some money (field int amount). Accounts implement the method transact (int $\mathrm{x}$ ) which increments or decrements the amount. If the amount exceeds the threshold of 1000 , then the account turns into a SavingsAccount. Similarly, if the amount falls below the threshold then the account turns into a DailyAccount. Savings accounts pay interest. ${ }^{3}$

Class definitions may be preceded by the keyword state or root with the following meaning:

\footnotetext{
3 The example would have been expressed more naturally if we could use abstract classes, and exceptions, but these are not part of $\mathcal{F} i c k l e_{\text {II }}$.
} 


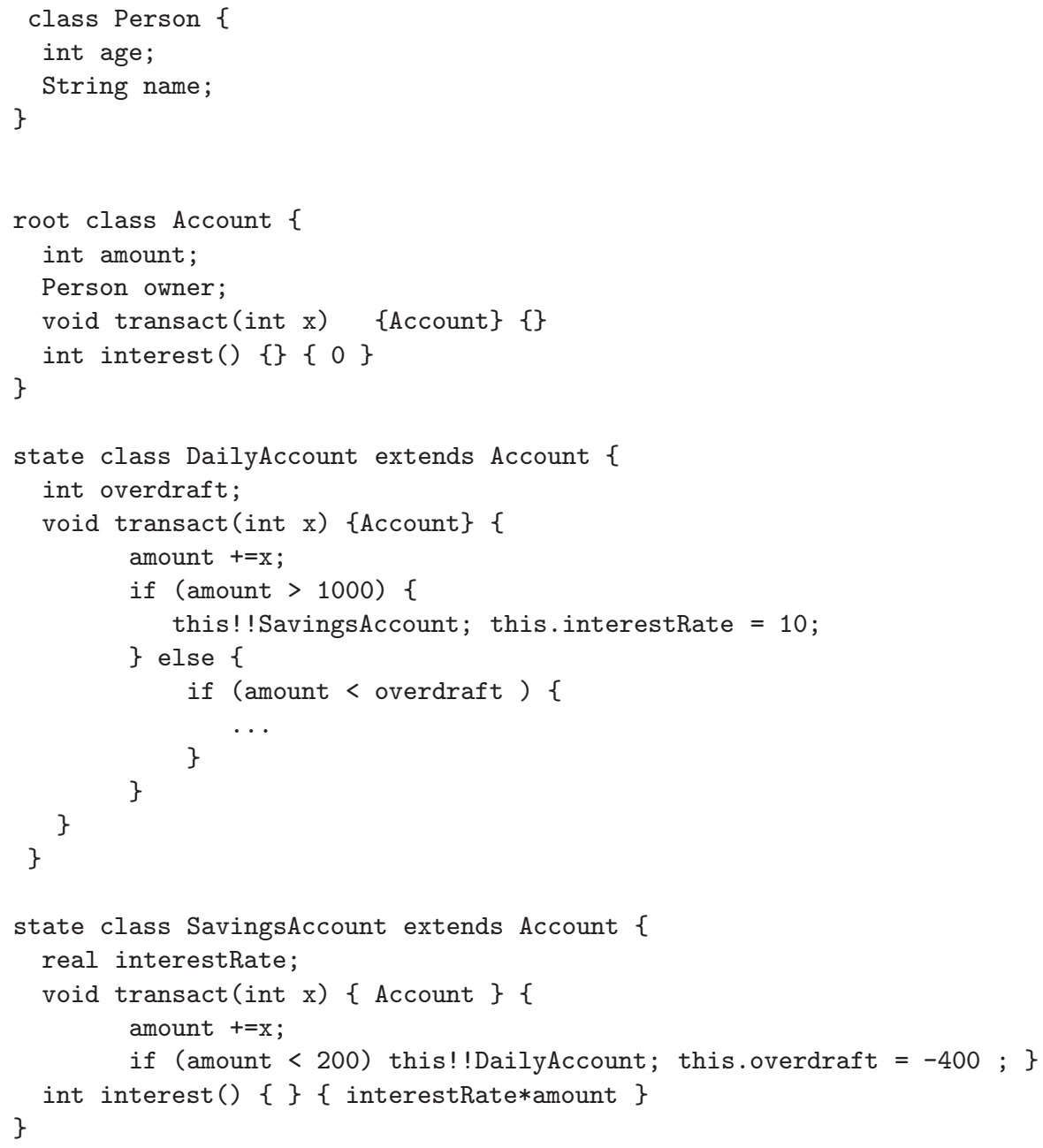

Fig. 1: Program Account - accounts with re-classifications 
- state classes describe the properties of an object while it satisfies some conditions; when it does not satisfy these conditions any longer, it can be explicitly re-classified to another state class. For example, DailyAccount describes daily accounts; when these hold more than 1000 drachmas, then they are re-classified to SavingsAccount.

We require state classes to extend either root classes or state classes.

- root classes abstract over state classes. Objects of a state class C1 may be re-classified to a class $\mathrm{C} 2$ only if $\mathrm{C} 2$ is a subclass of the uniquely defined root superclass of C1. For example, Account abstracts over DailyAccount and SavingsAccount; objects of class DailyAccount may be re-classified to SavingsAccount, and vice versa.

We require root classes to extend only non-root and non-state classes.

Objects of a non-state, non-root class $\mathrm{C}$ behave like regular Java objects, that is, are never re-classified. However, since state classes can be subclasses of non-state, non-root classes, an object bound to a variable $\mathrm{x}$ of type $\mathrm{C}$ may be re-classified. Namely, if C had two state subclasses C1 and C2 and x referred to an object $o$ of class $\mathrm{C} 1$, then $o$ may be re-classified to $\mathrm{C} 2$.

Objects are created in the usual way, by the expression new $\mathrm{C}()$, regardless of whether $\mathrm{C}$ is a state, or root, or non-state and non-root, class.

A re-classification statement has the form id!!C, and sets the class of id to $\mathrm{C}$, where $\mathrm{C}$ must be a state class with the same root class of the static type of id. The re-classification operation preserves the types and the values of the fields defined in the root class, removes the other fields, and adds the fields of $\mathrm{C}$ that are not defined in the root class, initializing them in the usual way. Re-classifications may be caused by re-classification statements, e.g., this!!SavingsAccount in method transact in class DailyAccount, or, indirectly, by method calls, like a.transact $(\ldots.){ }^{4}$ At the start of method transact of class DailyAccount the receiver is an object of (a subclass of) DailyAccount, therefore it has the fields amount, owner and overdraft, while it does not have the field interestRate. After execution of this! ! SavingsAccount the receiver is of class SavingsAccount, the fields amount and owner retain their values, the field overdraft disappears, and the field interestRate becomes available.

Fields must have declared types which are not state classes; we call these types non-state types. Thus, fields may denote objects which do change class, but these changes do not affect their type. Instead, the type of this, a parameter or a local variable may be a state class and may change.

Annotations like \{\} and $\{$ Account $\}$ before the method bodies $^{5}$ are called effects. Similarly to exceptions in throws clauses, effects list the root classes of all objects that may be re-classified by execution of that method. Methods annotated with the empty effect \{\} , like interest, do not cause any re-

\footnotetext{
${ }^{4}$ In the example, only this is re-classified; note that $\mathcal{F}$ ickle ${ }_{\text {II }}$ also allows reclassification of parameters and local variables.

${ }^{5}$ In a fully developed language these would appear before or immediately after the throws clauses.
} 
classification. Methods annotated with non-empty effects, e.g., transact annotated with $\{$ Account $\}$, may re-classify objects of (a subclass of) a class in their effect - in the example, of Account.

A method annotated with effects can be overridden only by methods annotated with the same or fewer effects ${ }^{6}$. By relying on effects annotations, the type and effect system of $\mathcal{F}_{i c k l e}$ II ensures that re-classifications will not cause accesses to fields or methods that are not defined for the object.

\section{The language $\mathcal{F}$ ickle $e_{\text {II }}$, a formal description}

\subsection{Syntax}

The syntax of $\mathcal{F}_{i c k l e}$ II is specified in Fig. 2. We use standard extended BNF, where a [ - ] pair means optional, $A^{*}$ means zero or more repetitions of $A$, and $A^{+}$ means one or more repetitions of $A$. We follow the convention that non terminals appear as nonTerm and terminals appear as term. We omit separators like "; " or "," where they are obvious (note that "; " in $\mathcal{F} i c k l e_{\mathrm{II}}$ is a separator rather than a terminator as in Java).

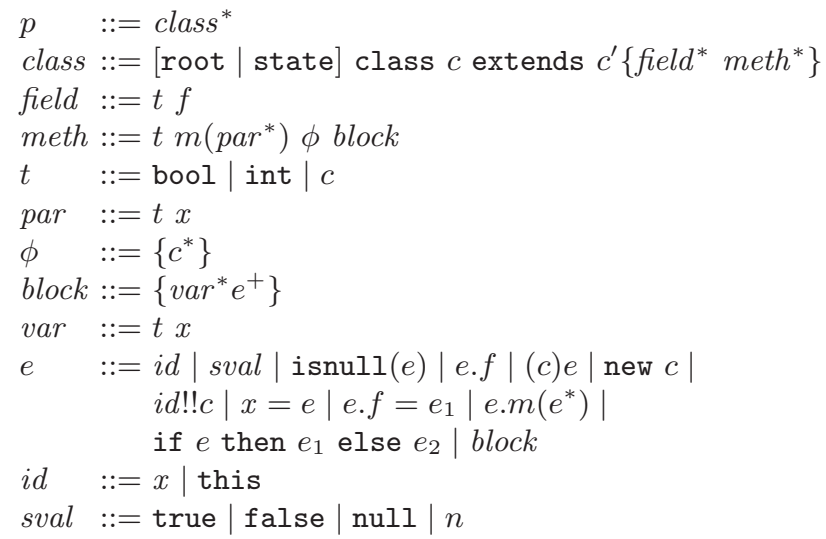

Fig. 2: Fickle $_{\text {II }}$ syntax

\footnotetext{
${ }^{6}$ This means that adding a new effect in a method of a class $c$ does not require any change to the subclasses of $c$, but may require some changes to its superclasses, and the classes using them. Note also that effects are explicitly declared by the programmer rather than inferred by the compiler. Even though effects inference could be implemented in practice, more flexibility in method overriding can be achieved by requiring the programmer to annotate methods with more effects than those that would be inferred (similarly to what happens with throws clauses for exceptions).
} 
Metavariables $c, f, m$ and $x$ range over sets of class names, field names, method names and variables, respectively. We assume a distinguished class name Object which cannot be used as name of a declared class.

A program is a sequence of class definitions. A class definition may be preceded by the keyword root or state. As already explained, state classes describe the properties of an object while it satisfies some conditions, whereas root classes abstract over state classes. ${ }^{7}$ Any subclass of a state or a root class must be a state class. Objects of a state class $c$ may be re-classified to class $c^{\prime}$, where $c^{\prime}$ must be a subclass of the uniquely defined root superclass of $c$.

A class specifies its superclass and declares a sequence of fields and methods. The type of fields may be either a primitive type or a non-state class; we call such types non-state types. Thus, fields may point to objects which change class, but these changes do not affect their type. In contrast, the type of identifiers (this, parameters and local variables) may be a state or root class.

Method declarations have the shape:

$$
\text { tm }\left(t_{1} x_{1}, \ldots, t_{q} x_{q}\right)\left\{c_{1}, \ldots, c_{n}\right\} \text { block }
$$

where $t$ is the result type, $m$ the name, $t_{1}, \ldots, t_{q}$ are the types of the formal parameters $x_{1}, \ldots, x_{q}$, and block is the body. The effect consists of root classes $c_{1}, \ldots, c_{n}$, with $n \geq 0$.

A block consists of a possibly empty sequence of local variable declarations and a sequence of expressions. Expressions include identifiers (that is, this, parameters and local variables), source language values (that is, constants of primitive types and null), field selection, casting, object creation, re-classification, assignment to a variable, assignment to a field, method call, conditional and block.

In object creation new $c, c$ may be any class, including a state class. Reclassification expressions, $i d ! ! c$, set the class of $i d$ to $c-c$ must be a state or a root class.

We require the inheritance hierarchy to be a tree, root classes to extend only non-root and non-state classes, and state classes to extend either root classes or state classes.

\subsection{Operational semantics}

We give a structural operational semantics that rewrites pairs of expressions and stores into pairs of values, exceptions, or errors, and stores - in the context of a given program $p$. The signature of the rewriting relation $\leadsto$ is:

\footnotetext{
${ }^{7}$ Notice that our proposal is orthogonal to the "abstract superclass rule" discussed in [15]. In fact, root classes are not necessarily abstract classes, and state classes may be superclasses only of other state classes.
} 


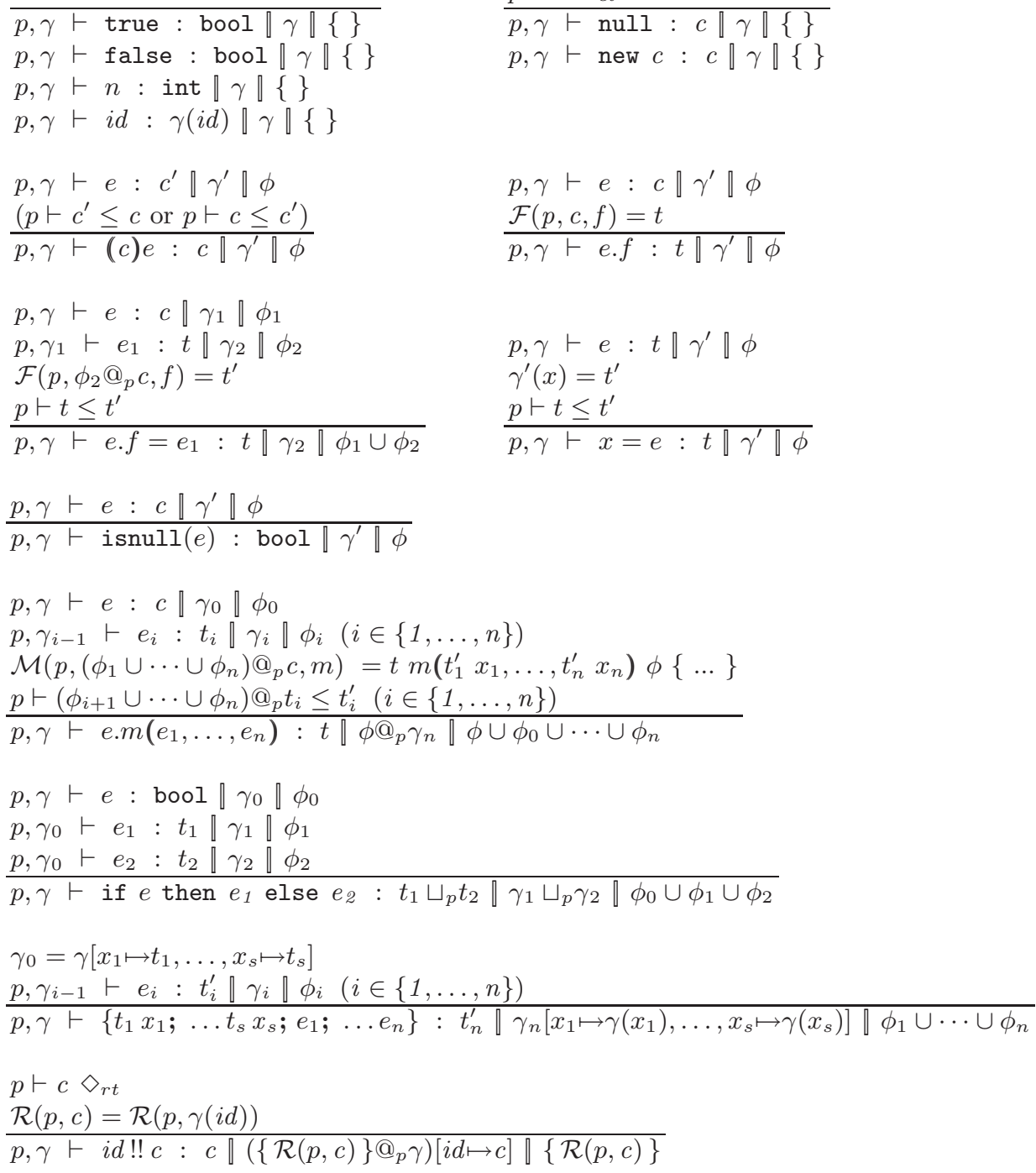

Fig. 3: $\mathcal{F}_{i c k l e}$ II - typing rules for expressions 


$$
\begin{aligned}
& \leadsto \quad p \longrightarrow \text { store } \longrightarrow(\text { val } \cup \text { dev }) \times \text { store } \\
& \text { store }=(\{\text { this }\} \longrightarrow \text { addr }) \cup(x \longrightarrow \text { val }) \cup \quad(\text { addr } \longrightarrow \text { object }) \\
& \text { val }= \text { sval } \cup \text { addr } \\
& \text { dev }=\{\text { nullPntrExc, castExc, stuckErr }\} \\
& \text { object }=\left\{\left[\left[f_{1}: v_{1}, \ldots, f_{r}: v_{r}\right]\right]^{c} \mid f_{1}, \ldots, f_{r}\right. \text { are field identifiers, } \\
&\left.v_{1}, \ldots, v_{r} \in \text { val, and } c \text { is a class name }\right\}
\end{aligned}
$$

The store is a finite partial function with finite domain, which maps this to an address, variables to values, and addresses to objects. Values are the source language values in Section 3.1, or addresses. Addresses may point to objects, but not to other addresses, primitive values, or null. Thus in $\mathcal{F}_{i c k l e}$ II , as in Java, pointers are implicit, and there are no pointers to pointers. As we will show, the execution of a well-typed expression does never produce a stuck error, although it may throw a null pointer exception or a cast exception. We denote stores with $\sigma$, and addresses with $\iota$.

We need some operations on objects and stores.

For object $o=\left[\left[f_{1}: v_{1}, \ldots, f_{l}: v_{l}, \ldots, f_{r}: v_{r}\right]\right]^{c}$, store $\sigma$, value $v$, address $\iota$, identifier or address $z$, field identifier $f$, value or object $w$, we define:

$$
\begin{array}{ll}
\text { - field access } & o(f)= \begin{cases}v_{l} & \text { if } f=f_{l} \text { for some } l \in 1, \ldots, r, \\
\mathcal{U} d f & \text { otherwise }\end{cases} \\
\text { - object update } & o[f \mapsto v]=\left\{\begin{array}{l}
{\left[\left[f_{1}: v_{1}, \ldots, f_{l}: v, \ldots, f_{r}: v_{r}\right]\right]^{c}} \\
\text { if } f=f_{l} \text { for some } l \in 1, \ldots, r, \\
\mathcal{U} d f \text { otherwise }
\end{array}\right. \\
\text { - store update } & \sigma[z \mapsto w](z)=w, \quad \sigma[z \mapsto w]\left(z^{\prime}\right)=\sigma\left(z^{\prime}\right) \text { if } z^{\prime} \neq z .
\end{array}
$$

Also, we follow the convention that $\sigma(\iota)(f)=\mathcal{U} d f$ whenever $\sigma(\iota)=\mathcal{U} d f$.

Figures 14, 15, 16, and 17 from Appendix A list all the rewrite rules of $\mathcal{F}_{i c k l e}$ II. We discuss the two most significant rewrite rules of $\mathcal{F}_{i c k l e}$ II method call and re-classification.

For method calls, e.m $\left(e_{1}, \ldots, e_{n}\right)$, we evaluate the receiver $e$, obtaining an address, say $\iota$. We then evaluate the arguments, $e_{1}, \ldots, e_{n}$. We find the appropriate body by looking up $m$ in the class of the object at address $\iota$ - we use the term $\mathcal{M}(p, c, m)$ that returns the definition of method $m$ in class $c$ going through the class hierarchy in $p$, if needed (see Appendix A). We execute the body after substituting this with the current object, and assigning to the formal parameters the values of the actual parameters. After the call, we restore the receiver and parameters to the values they had immediately before execution of the body. ${ }^{8}$

For re-classification expressions, $i d ! ! d$, we find the address of $i d$, which must point to an object, say of class $c$. We replace the original object by a new object of

\footnotetext{
${ }^{8}$ We restore the references, but not the contents: thus, after a method call the side effects caused by execution of the method body survive. Note also that if one of the method parameters was undefined before the call, then it will be undefined after the call as well.
} 
class $d$. We preserve the fields belonging to the root superclass of $c$ and initialize the other fields of $d$ according to their types. The term $\mathcal{R}(p, t)$, defined by

$$
\mathcal{R}(p, t)=\left\{\begin{array}{l}
c \text { if } t \text { is a state class and } c \text { is the root superclass of } t \\
t \text { otherwise, }
\end{array}\right.
$$

denotes the least superclass of $t$ which is not a state class, if $t$ is a class, and denotes $t$ itself if $t$ is not a class or a non-state class. Moreover, $\mathcal{F} s(p, c)$ denotes the set of all fields (either directly defined or inherited) of class $c$, and $\mathcal{F}(p, c, f)$ the type of field $f$ in class $c$ (see Appendix A). Note that we do not allow hiding of fields in $\mathcal{F i c k l e}_{\text {II }}{ }^{9}$

$$
\begin{aligned}
& \mathcal{C}(p, c)=[\text { root } \mid \text { state }] \text { class } c \text { extends } c^{\prime}\{\ldots\} \\
& \forall f: \mathcal{F D}(p, c, f)=t_{f} \quad \Longrightarrow \quad p \vdash t_{f} \diamond_{f t} \quad \text { and } \quad \mathcal{F}\left(p, c^{\prime}, f\right)=\mathcal{U} d f \\
& \forall m: \mathcal{M D}(p, c, m)=t m\left(t_{1} x_{1}, \ldots, t_{n} x_{n}\right) \phi \text { block } \Longrightarrow \\
& p \vdash \phi \diamond \\
& p, t_{1} x_{1}, \ldots, t_{n} x_{n}, c \text { this } \vdash \text { block }: t^{\prime} \rrbracket \gamma^{\prime} \rrbracket \phi^{\prime} \\
& p \vdash t^{\prime} \leq t \\
& \phi^{\prime} \subseteq \phi \\
& \mathcal{M}\left(p, c^{\prime}, m\right)=\mathcal{U} d f \text { or }\left(\mathcal{M}\left(p, c^{\prime}, m\right)=t m\left(t_{1} x_{1}, \ldots, t_{n} x_{n}\right) \phi^{\prime \prime}\{\ldots\} \text { and } \phi \subseteq \phi^{\prime \prime}\right) \\
& \vdash p \diamond_{h} \\
& \frac{\forall c: \quad \mathcal{C}(p, c) \neq \mathcal{U} d f \quad \Longrightarrow \quad p \vdash c \diamond}{\vdash p \diamond}
\end{aligned}
$$

Fig. 4: $\mathcal{F}_{i c k l} e_{\text {II }}$ - rules for well-formed classes and programs

\section{$3.3 \quad$ Typing}

Widening, environments, effects The following assertions, defined in Fig. 19 of Appendix A, describe kinds of classes, and the widening relationship between types:

$-p \vdash c \diamond_{c t}$ means that $c$ is any class,

\footnotetext{
${ }^{9}$ In well-typed programs, $\mathcal{R}(p, c)=\mathcal{R}(p, d)$ always holds, and $c$ and $d$ must be state or root classes. This implies that re-classification depends only on the target class $d$, not on the class $c$ of the receiver. Therefore, a compiler could fold the type information into the code, by generating specific re-classification code for each state class. The rule for re-classification uses the types of the fields to initialize the fields, similarly to the rule for object creation.
} 
$-p \vdash c \diamond_{r t}$ means that $c$ is a re-classifiable type, i.e., either a root or a state class,

$-p \vdash t \diamond_{f t}$ means that $t$ is a non-state type, i.e., bool, or int, or a non-state class, and

$-p \vdash t \leq t^{\prime}$ means that type $t^{\prime}$ widens type $t$, i.e., $t$ is a subclass of, or identical to, $t^{\prime}$.

Environments, $\gamma$, map parameter names and local variables to types, and the receiver this to a class. They have the form $x_{1}: t_{1}, \ldots, x_{n}: t_{n}$, this : $c$. Lookup, $\gamma(i d)$, and update, $\gamma[i d \mapsto t]$, have the usual meaning, and are defined in Fig. 20 of Appendix A.

An effect, $\phi$, is a set $\left\{c_{1}, \ldots, c_{n}\right\}$ of root classes; it means that any object of a subclass of $c_{i}$ (including $c_{i}$ itself) may be re-classified. The empty effect, \{\} , guarantees that no object is re-classified. Effects are well-formed, i.e., $p \vdash$ $\left\{c_{1}, \ldots, c_{n}\right\} \diamond$, iff $c_{1}, \ldots, c_{n}$ are distinct root classes. Thus, $p \vdash\left\{c_{1}, \ldots, c_{n}\right\} \diamond$ implies that $c_{i}$ are not subclasses of each other.

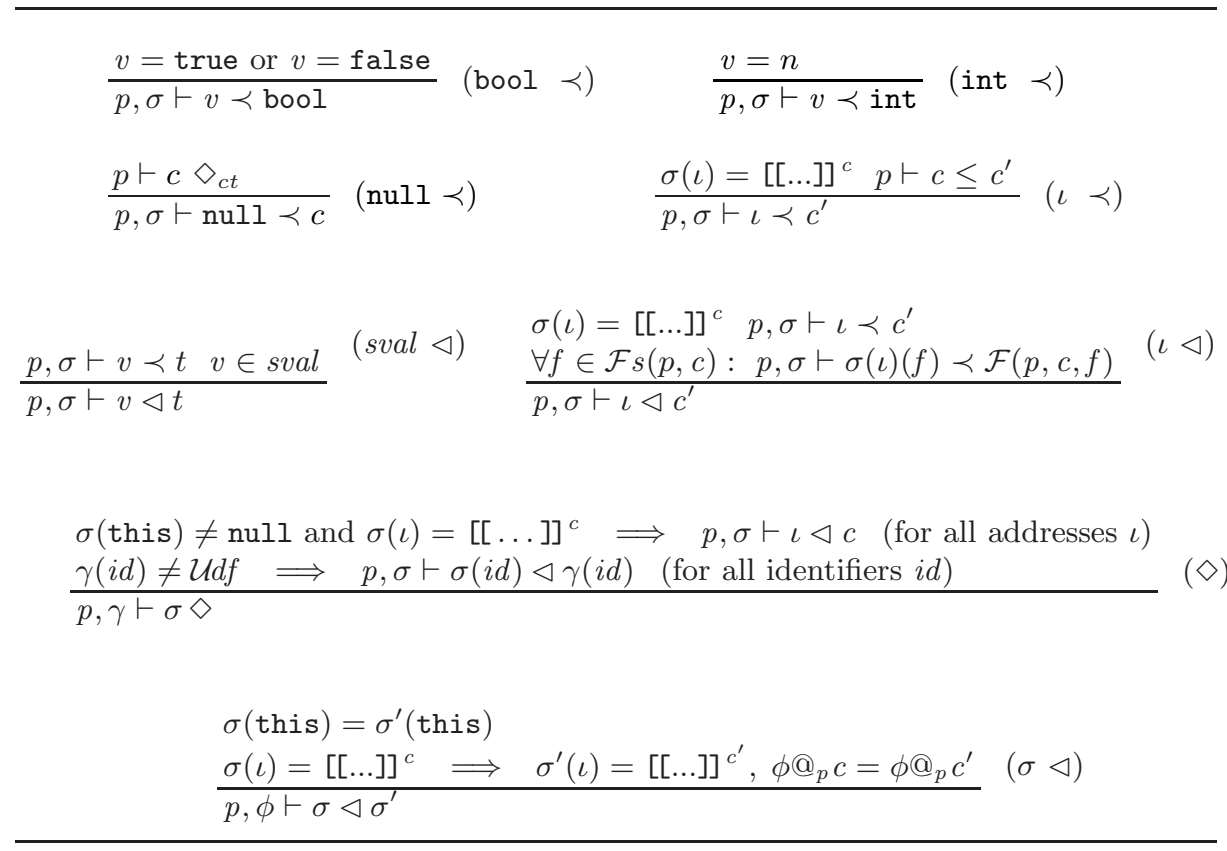

Fig. 5: Agreement between programs, stores, and values

Typing rules The typing rules are given in Fig. 3. We use the look-up functions $\mathcal{F}$ and $\mathcal{M}$, which search for fields and methods through the class hierarchy (see Appendix A). In the rules, $t \sqcup_{p} t^{\prime}$ is the least upper bound of $t$ and $t^{\prime}$ w.r.t. the 
$\leq$ relation between types in $p$, and $\gamma \sqcup_{p} \gamma^{\prime}$ associates with an identifier the least upper bound in $p$ of its types in $\gamma$ and $\gamma^{\prime}$. (The formal definitions can be found in Fig. 20 of Appendix A.) Moreover, we define the application of effects to types:

$$
\left\{c_{1}, \ldots, c_{n}\right\} @_{p} t= \begin{cases}c_{i} & \text { if } \mathcal{R}(p, t)=c_{i} \text { for some } i \in 1, \ldots, n \\ t & \text { otherwise }\end{cases}
$$

Note that, if

$$
p, \gamma \vdash e: t \rrbracket \gamma^{\prime} \rrbracket \phi
$$

is a derivable judgment, then the environments $\gamma$, and $\gamma^{\prime}$ are defined for the same set of identifiers, and any differences in the types associated with an identifier in $\gamma$ and $\gamma^{\prime}$ are due to the effect $\phi$. So, if $e$ is a $\mathcal{F} i c k l e_{\text {II }}^{-}$expression, then $\gamma=\gamma^{\prime}$ and $\phi=\{\}$.

Well-formed Programs A program is well formed (written $\vdash p \diamond$ ) if the inheritance hierarchy is well-formed $\left(\vdash p \diamond_{h}\right)$ and all its classes are well-formed $(p \vdash c \diamond)$ : Methods may override superclass methods only if they have the same name, argument, and result type, and their effect is a subset of that of the overridden method. Method bodies must be well formed, return a value appropriate for the method signature, and their effect must be a subset of that in the signature. See Fig. 4, where $\mathcal{C}(p, c)$ returns the definition of class $c$ in program $p$, and the look-up functions $\mathcal{F D}(p, c, f), \mathcal{M D}(p, c, m)$ search for fields and methods only in class $c$ (see Appendix A).

Soundness The main property of the type system is type soundness. This result will be needed in the proof of correctness of the translation.

In Fig. 5 we introduce the agreement relations between programs stores and values.

$-p, \sigma \vdash v \triangleleft t$ means that value $v$ has type $t$ in $p, \sigma$. When the value is an address $\iota$, then $t$ must be a class type $c$, and the store $\sigma$ must map $\iota$ to an object of class $c$ whose fields have the right type. The definition of $p, \sigma \vdash v^{\prime} \prec t^{\prime}$ is needed to break the circularity of the definition, since the value of one of the fields of the object could be $\iota$.

$-p, \gamma \vdash \sigma \diamond$ means that: for all addresses $\iota$, if $\sigma(\iota)=[[\cdots]]^{c}(c$ is the class of the object to which $\iota$ is mapped in $\sigma$, then $\iota$ has type $c$ in $p, \sigma$ (see the previous definition). Moreover, the value of the identifiers and of this in the store $\sigma$ agree with their type in the environment $\gamma$.

$-p, \phi \vdash \sigma \triangleleft \sigma^{\prime}$ means that store $\sigma^{\prime}$ is the store that may be obtained from $\sigma$ after the evaluation of an expression whose effects are $\phi$. So the address bound to this is not changed, and only objects that may have being reclassified are the objects that have class which is a subclass of one of the classes in $\phi$. 
The following theorem asserts that the evaluation of a well-typed expression in a store that agrees with the typing environment, results either in an exception, or in a value that is of the right type. Moreover, the store resulting from the evaluation agrees with the environment resulting from the typing judgment. That is the evaluation of a well-typed expression cannot produce stuckErr. The proof of the theorem can be found in [12].

Theorem 1. Let $p, \gamma \vdash e: t \rrbracket \gamma^{\prime} \rrbracket \phi$. If $p, \gamma \vdash \sigma \diamond$ and $e, \sigma \underset{p}{\longrightarrow} w, \sigma^{\prime}$, then

$-w=v, p, \gamma^{\prime} \vdash \sigma^{\prime} \diamond, p, \sigma^{\prime} \vdash v \triangleleft t$, and $p, \phi \vdash \sigma \triangleleft \sigma^{\prime}$, or

$-w \in\{$ castExc, nullPntrExc $\}$.

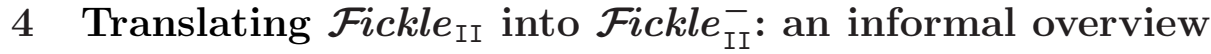

In this section we give an informal overview of the translation; we outline the encoding of objects (Section 4.1), and then discuss an example (Section 4.2).

\subsection{Encoding of objects}

The translation is based on the idea that each object $o$ of a state class $c$ can be encoded in $\mathcal{F}_{i c k l e_{\text {II }}^{-}}$by a pair $\langle i d, i m p>$ of objects; we call $i d$ the identity object of imp and imp the implementor object of $i d$. Roughly speaking, $i d$ provides the identity of $o$, and imp the behavior of $o$, so that any re-classification of $o$ changes $i m p$ but not $i d$ and method invocations are resolved by imp. Hence, two implementors paired with the same identity represent the same object at different execution stages.

An object $o$ which is not an instance of a state class does not need to be encoded in principle; however, for uniformity, the same kind of encoding described above is adopted also in this case, so that during the execution of a translated program there will be exactly an identity object for any $\mathcal{F}_{i c k l e}$ II object. Note that, while there could be more than one implementor encoding a $\mathcal{F}_{i c k l e}$ II object, say $\langle i d, i m p\rangle$ and $\left\langle i d, i m p^{\prime}\right\rangle$, the converse cannot be true: if $\langle i d, i m p\rangle$ and $\left\langle i d^{\prime}, i m p\right\rangle$ are pairs encodings $\mathcal{F}_{\text {ickle }}$ II objects, then $i d=i d^{\prime}$.

Re-classification of objects can be exemplified by the diagram in Fig. 6. As shown there, the identity object is the same during the lifetime of a $\mathcal{F i c k l e}_{\mathrm{II}}$ object, whereas at different times the implementor object can change; the right implementor can always be recovered by the imp field of the identity object (dotted lines in the figure show obsolete values of this field).

Classes are translated according to the following two rules:

- each $\mathcal{F}_{i c k l e}$ II class (including Object) is translated into exactly one $\mathcal{F} i c k l e_{\text {II }}^{-}$ class (whose instances are implementors);

- the translation preserves the inheritance hierarchy.

We illustrate the above in terms of the classes in Example 1.

Example 1. The following $\mathcal{F}_{\text {ickle }}$ II program defines the classes P, R, S1, and S2. 


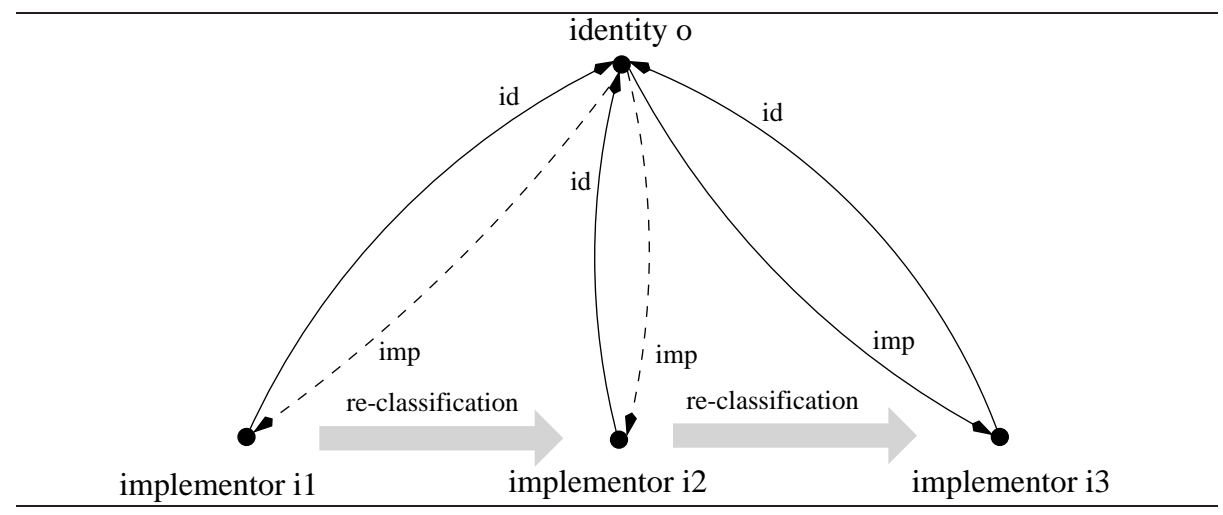

Fig. 6: Re-classification of objects

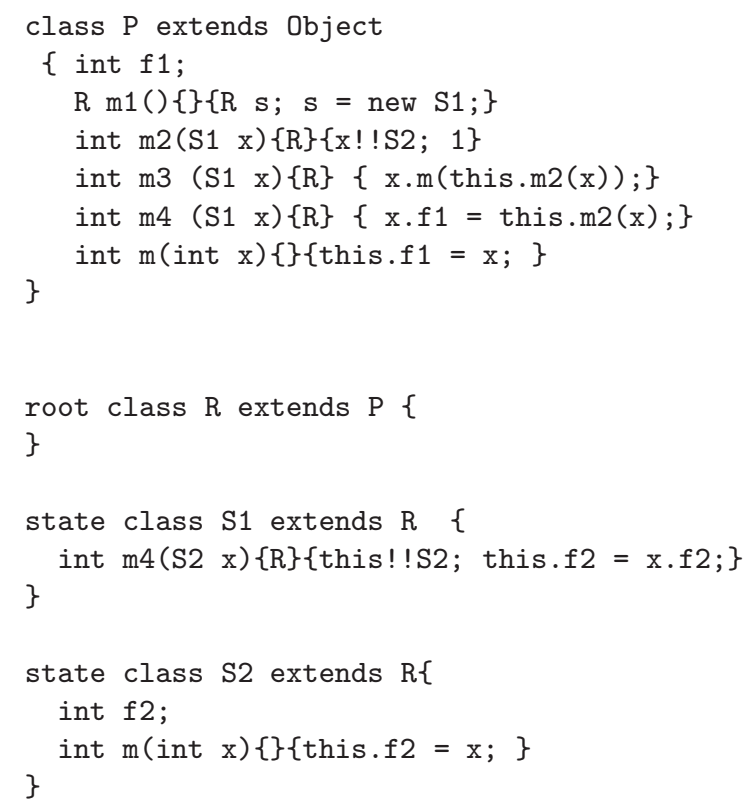

After the instruction

$\mathrm{s}=$ new $\mathrm{S} 1$;

in the body of $\mathrm{m} 1$, the $\mathcal{F} i c k l e_{\text {II }}$ object referred by $\mathrm{s}$ is encoded in the translation, as sketched in Fig. 7, by the two $\mathcal{F} i c k l e_{\text {II }}^{-}$objects $o$ and $o 1$ in which the field imp of $o$ points to $o 1$ and the field id of $o 1$ points to $o$.

The object $o 1$ has two fields: id of type Identity (inherited from class FickleObject, see Section 5.2), and $\mathrm{f} 1$ (inherited from P). The fields id and imp are used to recover the identity and the implementor of an object, respectively. In this case the field id points to the object $o$ of class Identity referred by s. 
s

$\mathrm{O}$

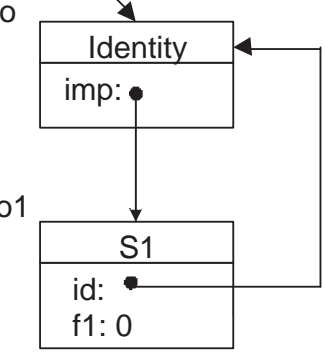

After executing: $\mathrm{s}=$ new $\mathrm{S} 1$

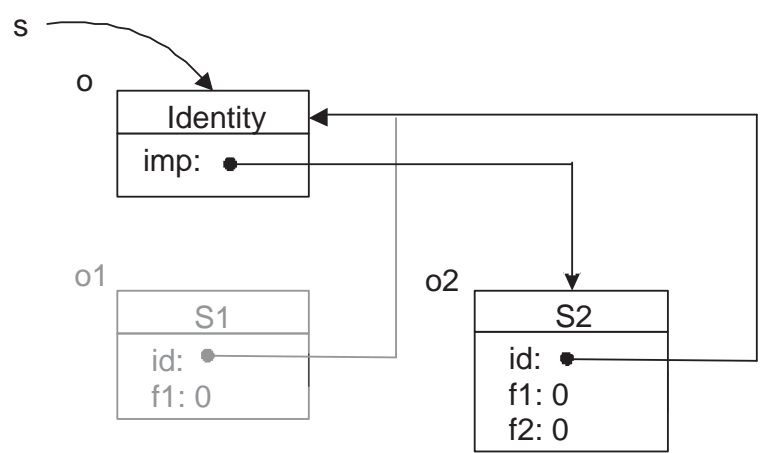

After executing:

$\mathrm{s}=$ new $\mathrm{S} 1 ; \mathrm{s} ! \mathrm{S} 2$

Fig. 7: Encoding of the $\mathcal{F}_{i c k l e}$ II object referred by s

Assume now that the object referred by $\mathrm{s}$ is re-classified to $\mathrm{S} 2$, e.g. through $\mathrm{s} ! ! \mathrm{S} 2$. Then, a new object, o2, of class $\mathrm{S} 2$ is created, and the field imp of the identity $o$ points to the new object $o 2$.

\subsection{An example of translation}

In this section we illustrate the translation of the classes in Example 1. Below we give the result of the translation of class $\mathrm{P}$, together with the definitions ${ }^{10}$ of classes Identity and FickleObject which are added in the translation of any program.

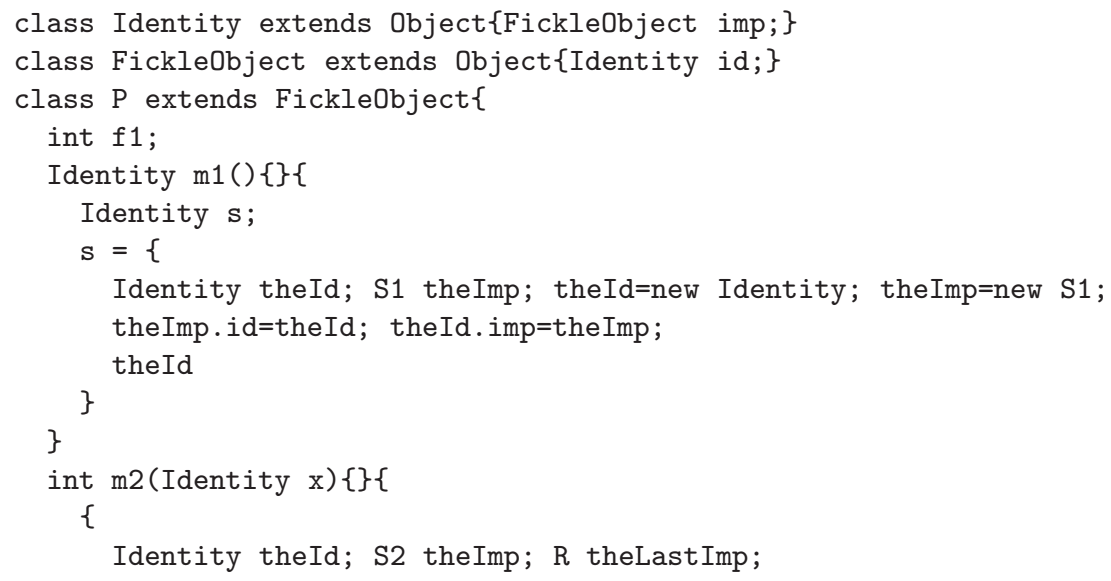

${ }_{10}$ the declarations are just the signatures in some cases 


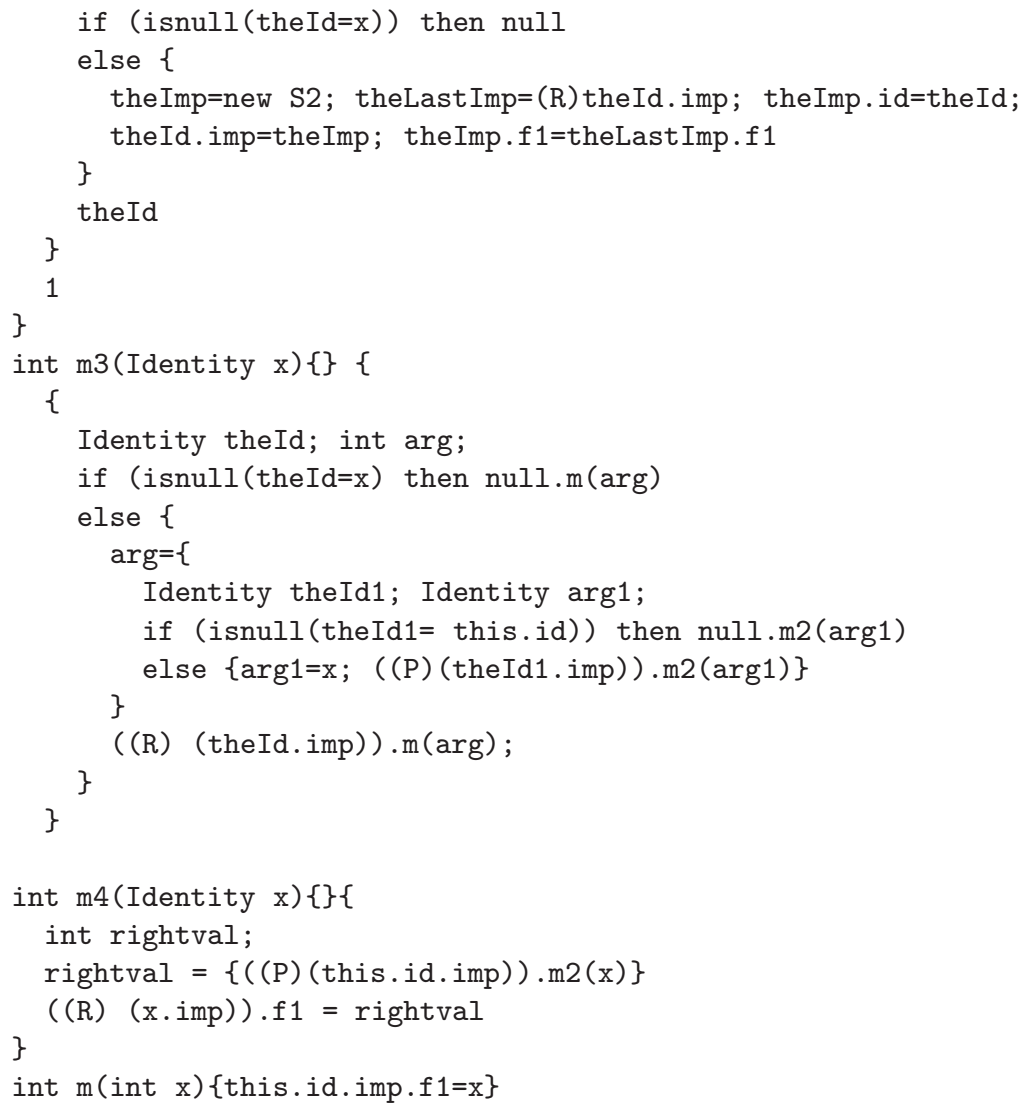

The translation maps the Fickle $_{\text {II }}$ class $\mathrm{P}$ into the Java class $\mathrm{P}$, which extends FickleObject, and hence inherits field Identity id.

We now consider the translation of method m1. First of all, note that type $\mathrm{R}$ in the result type of the method and in the local variable declaration is translated to Identity. Indeed, a property of the translation is that expressions of a class type are translated to expressions of type Identity (see Theorem 2).

The translation of the method body also demonstrates the encoding of the creation of a new object of class S1: As explained above, two new objects (the identity and the implementor) are created, which point to each other through their fields imp and id, respectively. Then, the identity object is returned.

The translation of method $\mathrm{m} 2$ demonstrates the encoding of the re-classification of the parameter $\mathrm{x}$ to S2. First of all, it is necessary to check whether $\mathrm{x}$ is null, since in this case the re-classification will have no effect. Otherwise, a new implementor object of class S2 is created and this implementor object and the identity object are made to point to each other. Moreover, all fields common to the new and old implementor object ${ }^{11}$, are copied from the old (theLastImp) to the new (theImp) implementor object.

The translation of method $\mathrm{m} 3$ demonstrates the encoding of method calls. Consider the external method call $\mathrm{x} . \mathrm{m}(\ldots)$. First, it is necessary to check that the receiver $\mathrm{x}$ is

${ }^{11}$ Common fields are those inherited from their most specific common superclass. 
not null. Then, the argument (which is in turn a method call this.m2(x)) is evaluated. Finally, the current implementor, theId.imp, of the receiver is selected and the method $\mathrm{m}$ is invoked on it. Note, that the implementor can be correctly selected only after the evaluation of the argument, because this evaluation could re-classify the receiver object. This is exactly what happens in this case: namely, the receiver is reclassified from $\mathrm{S} 1$ to $\mathrm{S} 2$, and thus the method $\mathrm{m}$ from class $\mathrm{S} 2$ has to be executed; if we selected the implementor earlier, then the method $\mathrm{m}$ from class $\mathrm{P}$ would be executed instead, which contravenes the $\mathcal{F}_{\text {ickle }}$ II semantics. This is the reason for the introduction of the auxiliary variables theId and arg. For the same reason, there is a cast to R (indeed, since evaluation of the argument could re-classify the receiver, we can only assume that the implementor has type $\mathrm{R}$ ).

The internal method call is translated in the same way; note that in this case the isnull test is useless, since the receiver is this, hence could be removed in an optimized translation. Note that the cast to $\mathrm{P}$ is necessary because the field imp has type FickleObject.

The translation of method $\mathrm{m} 4$ demonstrates the encoding of field assignment. The schema is analogous to that for method calls, except that here we have optimized the translation omitting unnecessary blocks, local variables and isnull tests. However, as in method $\mathrm{m} 3$, it is necessary to select the implementor only after evaluation of the right hand side of the assignment, because this evaluation could also re-classify the object containing the field; otherwise, 1 would be assigned to the field of the old implementor.

The translation of the method $\mathrm{m}$ has been optimized too, as well as the translation of the classes R, S1 and S2 below:

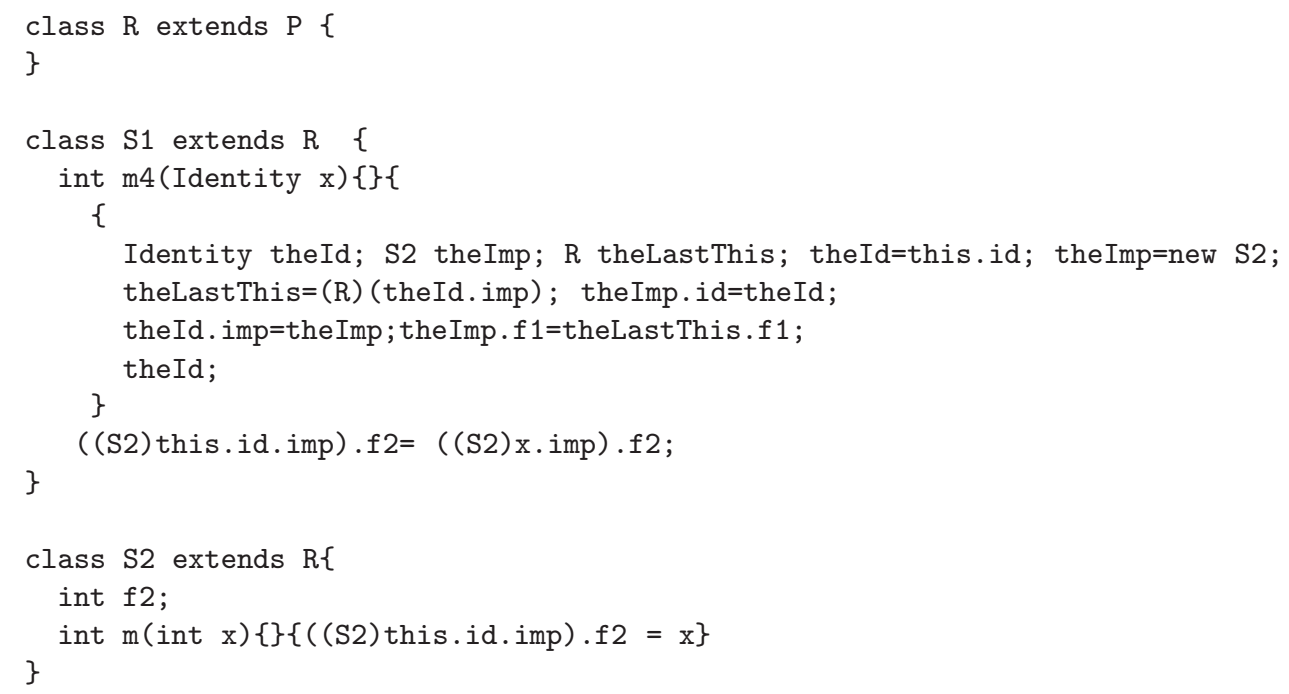

\section{Translation of $\mathcal{F}_{i c k l e}$ II into $\mathcal{F} i c k l e_{\text {II }}^{-}$: a formal definition}

In this section we give the formal definition of the translation. 


\subsection{Translation of programs}

The translation of a $\mathcal{F} i c k l e_{\text {II }}$ program $p$ consists of the declaration of the two special classes FickleObject and Identity, together with the translation of all classes declared in $p$. Since the translation of expressions depends on their types, the program $p$ is passed as parameter to the translation function for classes.

$$
\begin{aligned}
\llbracket p \rrbracket_{\text {prog }} \triangleq & \text { class Identity extends Object }\{\text { FickleObject imp; }\} \\
& \text { class FickleObject extends Object }\{\text { Identity id; }\} \\
& \llbracket \text { class }_{1} \rrbracket_{\text {class }}(p) \ldots \llbracket \text { class }_{n} \rrbracket_{\text {class }}(p), \quad \text { where } p=\text { class }_{1} \ldots \text { class }_{n}
\end{aligned}
$$

For simplicity, here we are implicitly assuming no name conflicts between the classes and fields declared in $p$ and the names FickleObject, Identity and $i^{12}$; however, such conflicts could be always avoided by a slightly more complex translation where class names and fields are suitably renamed.

\subsection{Translation of classes}

As previously said, each translated class extends class FickleObject. An object $o$ which needs to be re-classified to a state class $c$ (recall that in the translation every class except for Identity is subclass of FickleObject), and which is encoded by the pair $<i d, i m p\rangle$, is transformed into $\left\langle i d, i m p^{\prime}\right\rangle$, where $i m p^{\prime}$ denotes the new implementor of class $c$ (provided by a proper constructor of $c$; see definition below). Fields are initialized so that the identity and the new implementor point to each other. We introduce the translation of types.

Definition 1. Given a type $t$ and a class c define:

- theType $(t)=$ Identity if $t$ is a class, and theType $(t)=t$ otherwise, and

- theName $(c)=$ FickleObject if $c=$ Object, and theName $(c)=c$ otherwise.I replaced $c^{\prime}$ by $c$

Each $\mathcal{F}_{i c k l e}$ II class $c$ is translated into a single $\mathcal{F}_{i c k l e}-{ }_{\text {II }}^{-}$class containing the translation of all field and method declarations of $c$.

The translation of fields and methods is the same for any kind of class. Since the translation of expressions depends on their types, the program $p$ and the class $c$ defining the type of this is passed as parameter to the translation function for methods.

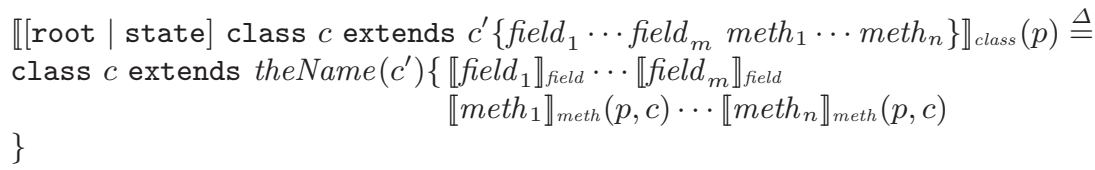

\subsection{Translation of field and variable (i.e. parameter or local variable) declarations}

$$
\begin{array}{ll}
\llbracket t & f \rrbracket_{\text {field }} \triangleq \text { theType }(t) f \\
\llbracket t & x \rrbracket_{\text {var }} \triangleq \operatorname{theType}(t) x
\end{array}
$$

$\overline{12}$ Field imp of class Identity does not conflict since no translated class extends Identity. 


\subsection{Translation of method declarations}

Translating methods consists of translating their bodies. Effects are omitted, whereas the signatures remain the same. Since the translation of expressions depends on their types, the program $p$ and the environment $\gamma$ defining the type of the parameters and of this must be passed as argument to the corresponding translation functions.

$$
\begin{gathered}
\llbracket t m\left(t_{1} x_{1}, \ldots, t_{n} x_{n}\right) \phi \text { block } \rrbracket_{\text {meth }}(p, c) \triangleq \\
\text { theType }(t) m\left(\llbracket t_{1} x_{1} \rrbracket_{\text {var }}, \ldots \llbracket t_{n} x_{n} \rrbracket_{\text {var }}\right)\{\} \llbracket b l o c k \rrbracket_{\text {expr }}(p, \gamma) \\
\text { where } \gamma=t_{1} x_{1}, \ldots, t_{n} x_{n}, c \text { this }
\end{gathered}
$$

\subsection{Translation of expressions}

Values, variables, this, null test, field selection, and cast In our encoding, in order to access the current implementor of an object we have to select the implementor currently pointed to by the identity of the object.

$$
\begin{aligned}
& \llbracket \operatorname{sval} \rrbracket_{e x p r}(p, \gamma) \triangleq \text { sval } \\
& \llbracket x \rrbracket_{\text {expr }}(p, \gamma) \triangleq x \\
& \llbracket \operatorname{this} \rrbracket_{\text {expr }}(p, \gamma) \triangleq \text { this.id } \\
& \llbracket \operatorname{isnull}(e) \rrbracket_{\text {expr }}(p, \gamma) \triangleq \operatorname{isnull}\left(\llbracket e \rrbracket_{\text {expr }}(p, \gamma)\right) \\
& \llbracket e . f \rrbracket_{\text {expr }}(p, \gamma) \triangleq\left((t h e N a m e(c))\left(\llbracket e \rrbracket_{e x p r}(p, \gamma) . \operatorname{imp}\right)\right) . f \\
& \text { where } p, \gamma \vdash e: c\left\|\gamma^{\prime}\right\| \phi .
\end{aligned}
$$

Downcasting to $c$ is needed because field imp has type FickleObject.

$$
\begin{aligned}
& \llbracket(c) e \rrbracket_{\text {expr }}(p, \gamma) \triangleq\{\text { Identity } x ; \\
& \text { if }\left(\operatorname{isnull}\left(x=\llbracket e \rrbracket_{\text {expr }}(p, \gamma)\right)\right) \\
& \text { then null } \\
& \text { else }((\text { theName }(c))(x . \text { imp })) \text {.id } \\
& \text { where } \gamma(x)=\mathcal{U} d f
\end{aligned}
$$

Variable assignment, field assignment, and method call Field $f$ of the object denoted by the translation of $e$ is accessed through the implementor of its identity. However, $e_{1}$ could re-classify the object, therefore the selection of field imp is correct only after evaluating the translation of $e_{1}$. This is achieved by introducing auxiliary local variables. The same idea is applied to the translation of method call.

$$
\begin{aligned}
& \llbracket x=e \rrbracket_{\text {expr }}(p, \gamma) \triangleq x=\llbracket e \rrbracket_{\text {expr }}(p, \gamma) \\
& \llbracket e . f=e_{1} \rrbracket_{e x p r}(p, \gamma) \triangleq\{\text { Identity } x ; \\
& \text { the Type }(t) x_{1} \text {; } \\
& \text { if }\left(\operatorname{isnull}\left(x=\llbracket e \rrbracket_{\text {expr }}(p, \gamma)\right)\right) \\
& \text { then null. } f=x_{1} \\
& \text { else }\left\{x_{1}=\llbracket e_{1} \rrbracket_{\text {expr }}\left(p, \gamma_{1}\right)\right. \text {; } \\
& \text { \} } \\
& \left(\left(\phi_{2} @_{p} \text { theName }(c)\right)(x . \mathrm{imp})\right) . f=x_{1}
\end{aligned}
$$

where $p, \gamma \vdash e: c\left\|\gamma_{1}\right\| \phi_{1}$ and $p, \gamma_{1} \vdash e_{1}: t\left\|\gamma_{2}\right\| \phi_{2}$, and $\gamma(x)=\gamma\left(x_{1}\right)=\mathcal{U} d f$. 


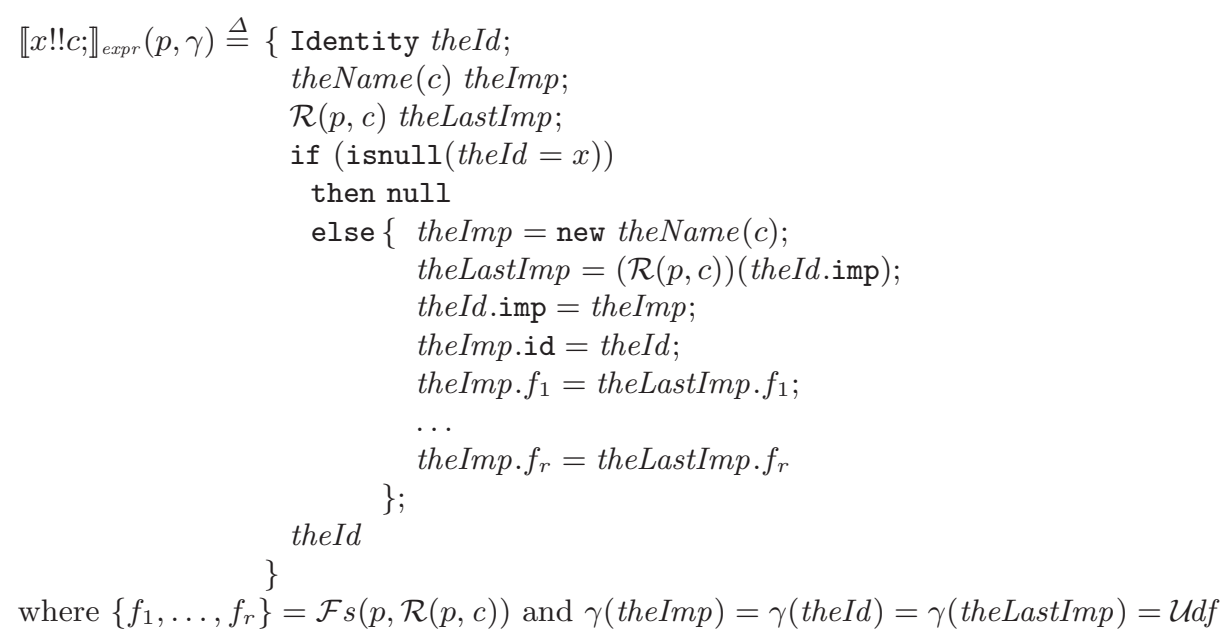

\section{Conditionals and blocks}

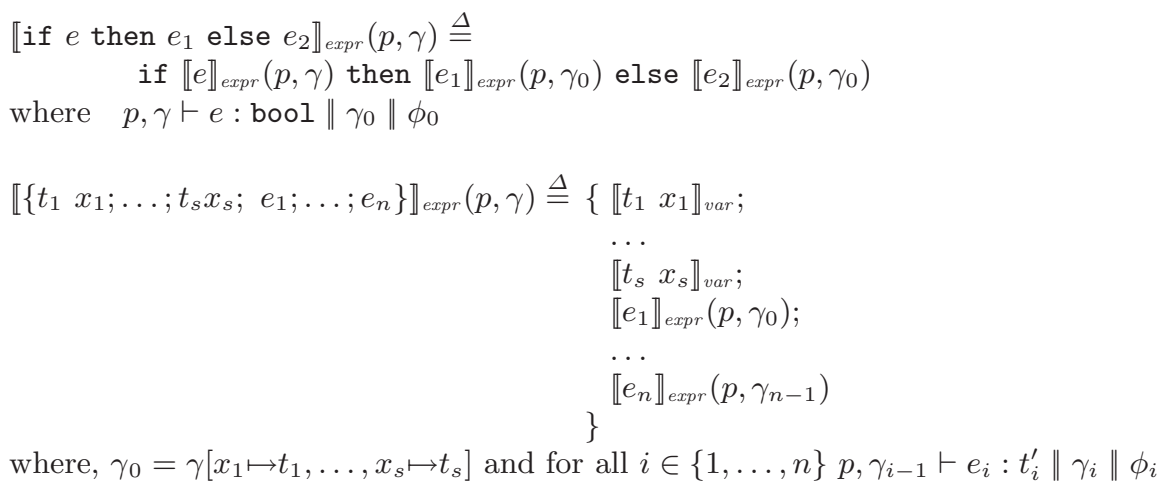

\section{Properties of the translation}

In this section we formalize and prove the good properties of the translation previously mentioned.

\subsection{Preservation of static semantics}

For any environment $\gamma$, its translation $\llbracket \gamma \rrbracket$ is defined by

$$
\llbracket \gamma \rrbracket=\{\operatorname{theType}(t) x \mid \gamma(x)=t\} \cup\{\operatorname{theName}(\gamma(\text { this })) \text { this }\} .
$$

Theorem 2. Let $p$ be a program s.t. $\vdash p \diamond, \gamma$, and $\gamma^{\prime}$ environments s.t. $p \vdash \gamma($ this $) \diamond_{c t}$, $e$ an expression, $t$ a type and $\phi$ an effect.

If $p, \gamma \vdash e: t \rrbracket \gamma^{\prime} \llbracket \phi$, then $\llbracket p \rrbracket_{\text {prog }}, \llbracket \gamma \rrbracket \vdash \llbracket e \rrbracket_{\text {expr }}(p, \gamma):$ theType $(t) \rrbracket \llbracket \gamma \rrbracket \llbracket\{\}$. 
Proof. See Appendix B.

Theorem 3. Let $p$ be a program s.t. $\vdash p \diamond$, and $c$ a class name. If $p \vdash c \diamond$, then $\llbracket p \rrbracket_{\text {prog }} \vdash c \diamond$.

Proof. See Appendix B.

Theorem 4. Let $p$ be a program. If $\vdash p \diamond$, then $\vdash \llbracket p \rrbracket_{\text {prog }} \diamond$.

Proof. See Appendix B.

\subsection{Preservation of dynamic semantics}

In this section we show that the dynamic semantics of expressions is preserved by the translation.

We introduce a relation between stores $p, \gamma \vdash \sigma \approx \sigma^{\prime}$ that expresses the fact that store $\sigma^{\prime}$ contains the "translation" of the objects in store $\sigma$. More precisely, an object $o$ of class $c$ in $\sigma$ is translated in $\sigma^{\prime}$ into an object of class Identity whose imp field points to an implementor object $o^{\prime}$ which is an instance of the translation of the class $c$. Values of identifiers in $\sigma$ are preserved in $\sigma^{\prime}$, except for this, whose value in $\sigma^{\prime}$ is (the address of) an implementor object whose id field points to the address which is the value of this in $\sigma$. The store $\sigma$ and $\sigma^{\prime}$ (except for this), are assumed to agree with the environments $\gamma$ and $\llbracket \gamma \rrbracket$, that is, they contain values which agree, w.r.t. typing, with their definitions (see Fig. 5 for the formal definition of $p, \gamma \vdash \sigma \diamond$ ). Regarding the agreement of this in store $\sigma^{\prime}$, observe that the store $\sigma^{\prime \prime}$ resulting from the evaluation of the translation of the re-classification of this, from class $c$ to class $d$, is such that $\sigma^{\prime \prime}$ (this) $=\iota$ and $\sigma^{\prime \prime}(\iota)=\left[\text { id : } \iota^{\prime} \cdots\right]^{c}$, (the translation of the expression does not contain re-classifications, whereas the original expression did) and $\sigma^{\prime \prime}\left(\sigma^{\prime \prime}\left(\iota^{\prime}\right)\right)($ imp $)=\left[\left[\text { id }: \iota^{\prime} \cdots\right]\right]^{d}$. So $\sigma^{\prime \prime}($ this $)$ does not agree with $d$ but agrees with $c$ which is the type of this before re-classification.

Definition 2. Let $p$ be a program, $\gamma$ an environment, $\sigma$ and $\sigma^{\prime}$ stores such that $p, \gamma \vdash$ $\sigma \diamond$ and $\llbracket p \rrbracket, \llbracket \gamma \rrbracket$ this $\mapsto c] \vdash \sigma^{\prime} \diamond$, for some $c$. We say that $\sigma^{\prime}$ is the translation of $\sigma$, and write $p, \gamma \vdash \sigma \approx \sigma^{\prime}$, if

1. $\sigma($ this $)=\sigma^{\prime}\left(\sigma^{\prime}(\right.$ this $\left.)\right)($ id $)$

2. for all $x, \gamma(x) \neq \mathcal{U} d f$ implies $\sigma(x)=\sigma^{\prime}(x)$, and

3. for all $\iota$, if $\sigma(\iota)=\left[\left[f_{1}: v_{1}, \ldots, f_{n}: v_{n}\right]\right]^{c}$ then

$-\sigma^{\prime}(\iota)=\left[\left[\text { imp : } \iota^{\prime}\right]\right]^{\mathrm{Id}}$,

$-\sigma^{\prime}\left(\iota^{\prime}\right)=\left[\left[i d: \iota, f_{1}: v_{1}, \ldots, f_{n}: v_{n}\right]\right]^{\operatorname{theName}(c)}$.

for a suitable renaming of the addresses in $\sigma^{\prime}$ (or $\sigma$ ).

We can now state the theorem that asserts that our translation is adequate. The proof of the theorem is in Appendix C.

Theorem 5. Let $e$ be an expression such that: $p, \gamma \vdash e: t \llbracket \gamma^{\prime} \llbracket \phi$, and $\sigma$, and $\sigma_{1}$, be stores such that $p, \gamma \vdash \sigma \approx \sigma_{1}$. Then

$$
e, \sigma \underset{p}{\longrightarrow} w, \sigma^{\prime} \quad \text { if and only if } \llbracket e \rrbracket, \sigma_{1} \underset{\llbracket p \rrbracket}{ } w, \sigma_{1}^{\prime}
$$

where either

$-w=v$, and $p, \gamma^{\prime} \vdash \sigma^{\prime} \approx \sigma_{1}^{\prime}$, or

- $w \in\{$ castExc, nullPntrExc $\}$.

Proof. See Appendix C. 


\section{Four design alternatives}

The design of the translation of $\mathcal{F}$ ickle is the outcome of several iterations. In this section we outline and compare these.

We first developed Version_1, which we implemented through Carmela, a Java program mapping $\mathcal{F} i c k l e^{\text {st }}$ onto Java [4]. $\mathcal{F} i c k l e^{\text {st }}$ is a statement oriented version of Fickle $_{\text {II }}$. The development of Carmela proved more complex than anticipated, and we thus started a formal treatment, which we continued after the development of the software. This work led to Version_2 [1]. We had then some further ideas for improvement, which lead to Version_3 [2]. Finally, we developed Version_4, the approach described in this paper. Version_4 has been implemented through Isabella [5], a Java program which maps $\mathcal{F}_{i c k l e}$ st onto Java.

All translations are based on the idea of pairs of $\langle i d, i m p>$ objects, which represent the identity and the implementor of the corresponding $\mathcal{F}$ ickle object. Starting from that basic idea, the following questions needed to be assessed:

- Would one translated object play both the role of the identity and implementor? Originally we allowed translated objects to take on the role of both the identity and implementor. This requires the object to have both id and imp fields. Later we separated the roles and each object was either an implementor or identity. Hence, each object contained either an id or imp field.

- Does the translation of a reference to a $\mathcal{F} i c k l e$ object refer to the identity or implementor part of the pair?

Having the representation of the $\mathcal{F} i c k l e$ object point to the implementor allows for a type preserving translation. However, it also allows references to objects that are "outdated". This reduces the garbage collection possibilities and increases the length of expressions required to reach the "active" implementation object. Referencing the identity simplifies expressions and allows for garbage collection of "outdated" objects.

- How are variables of state class type translated? In the original definition of $\mathcal{F}$ ickle only this could have state class type. Therefore, the early designs did not cater for that, and only the Version_ 4 does.

Figures 8, 9, 10 and 11 show the representation of the $\mathcal{F}$ ickle objects before and after re-classification in Version_1, Version_2, Version_3, and Version_4, respectively. The bold arrows represent references that exist between $\mathcal{F}$ ickle objects, whereas the normal arrows represent references introduced by the translation. Figures 12 and 13 summarize the differences between the various translations.

\subsection{Version_1}

$\mathcal{F}$ ickle objects may be represented through one or through a pair of objects, depending on their history. Namely, objects that have not been re-classified are represented by a single object. Implementor and identity objects belong to state subclasses of the root class. This follows because an object that has not yet been re-classified is both the implementor and identity. Both the identity and implementor objects contain a field id and imp.

Access to members of all classes goes through the indirection of id and imp. References to $\mathcal{F}$ ickle objects are represented through references to the implementor object, 
e.g.., the variable account in Fig. 8. Therefore, during run-time of the translated program references to "outdated" objects are possible: again in Fig. 8, account refers to an outdated object after the re-classfication.

The translation preserves types up to roots, i.e., the translation of a Fickle expression of type $t$ has type $\mathcal{R}(p, t)$. The translation is optimized in terms of type casts and number of objects created at run-time.

\section{$7.2 \quad$ Version_2}

Version_2 is a simplification over Version_1, in that all Fickle objects are represented through a pair of <id, imp $>$ objects. In contrast to Carmela, implementor objects belong to state classes, and identity objects belong to root classes. As in Version_1, identity objects contain a field id and implementor objects contain a field imp.

Access to members of all classes goes through the indirection of id and imp. Type casts are required when accessing members because the imp field has type FickleObject. FickleObject is the superclass of all translated $\mathcal{F}$ ickle classes. References to $\mathcal{F}$ ickle objects are represented through references to the identity object. References to "outdated" objects are not possible. This opens more possibilities for garbage collection.

This translation preserves types up to roots. Overall the translation is simpler, but less efficient than Version_1.

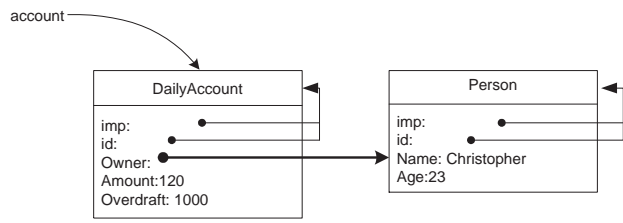

Before Re-classification

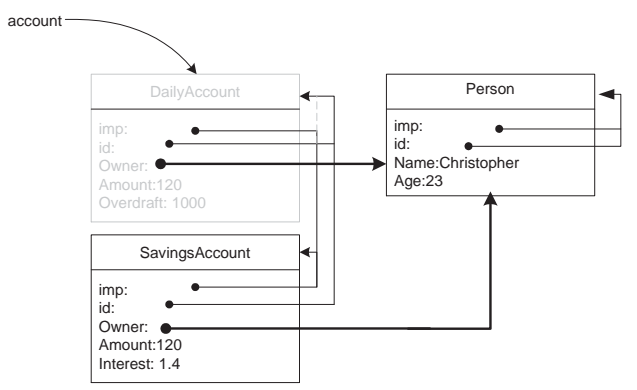

After Re-classification

Fig. 8: Objects in Version_1

\subsection{Version_3}

In Version_3 we realized that we could achieve a significant simplification over Version_2, by representing identities through objects of the same class, regardless of the root class of the $\mathcal{F}$ ickle object. Thus, we introduced the class Identity, which has the field imp, pointing to the implementor of class FickleObject. The translated $\mathcal{F}$ ickle classes contain a field id, pointing to objects of type Identity.

Access to members of all classes goes through the indirection of id and imp. Type casts are required when accessing members from all classes. References to $\mathcal{F}$ ickle objects 

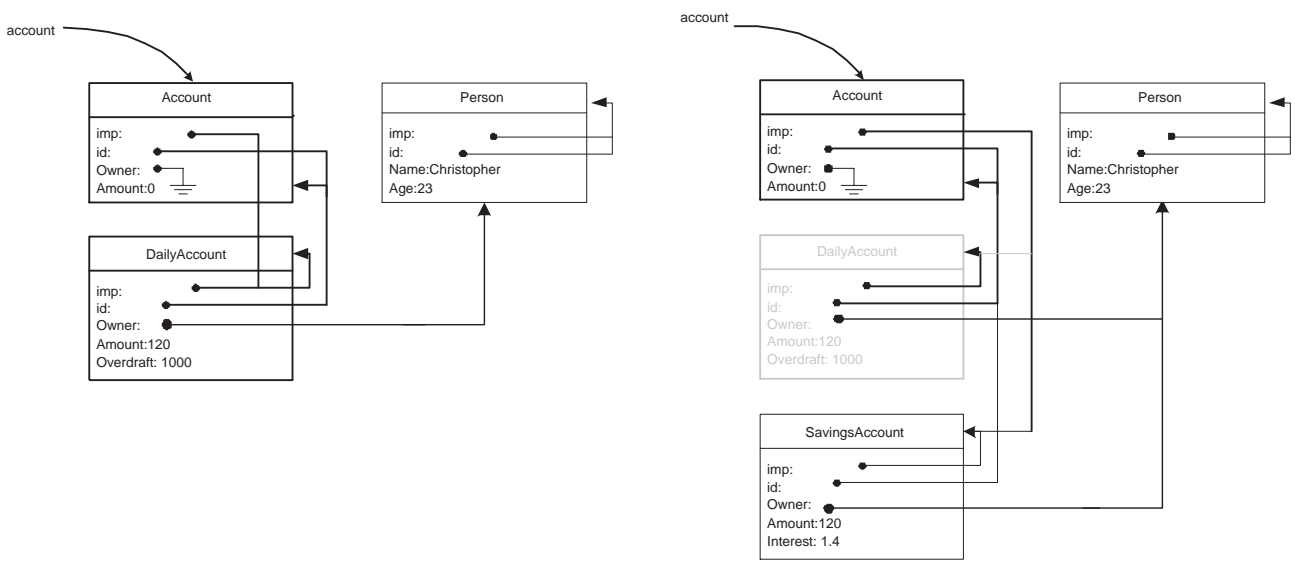

Before Re-classification

After Re-classification

Fig. 9: Objects in Version_2

are represented through references to the implementor object, and therefore references to "outdated" objects are possible.

The translation preserves types, i.e., the translation of a $\mathcal{F}$ ickle expression of type $t$ has type t. Compared with Version_2, Version_3 requires the same number of objects, but the identity objects are smaller than the corresponding objects of root class type in Version_2, as they only contain the field imp. Thus, the translation is simpler, and more efficient than Version_2.

\subsection{Version_4}

Finally, we realized that we could achieve a further simplification, by adopting the identity objects as they are in Version_3, and representing references to $\mathcal{F}_{\text {ickle }}$ objects though references to identity objects.

Access to members of all classes goes through the indirection of imp only, thus, requiring fewer access than any other translation. Type casts are required for all field accesses and method calls. Access to members of the receiver (this) is different because this is the only Fickle entity that is represented by an implementor rather than an identity object. Because this may be re-classified during a method activation, access to its members goes through the indirection of both id and imp. As in Version_2, because references to $\mathcal{F} i c k l e$ objects are represented through references to the identity, references to "outdated" objects are not possible, and thus more possibilities for garbage collection are open.

The translation does not preserve types, i.e., the translation of any $\mathcal{F}$ ickle expression of class type $c$ has type Identity.

Comparing Version_4 with Version_3, it requires the same number of objects, but fewer intermediate steps to represent field access and method call, and allows more opportunities to garbage collection. Therefore, Version_4 combines simplicity with efficiency. 


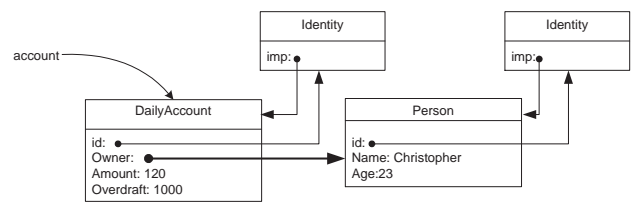

Before Reclassification

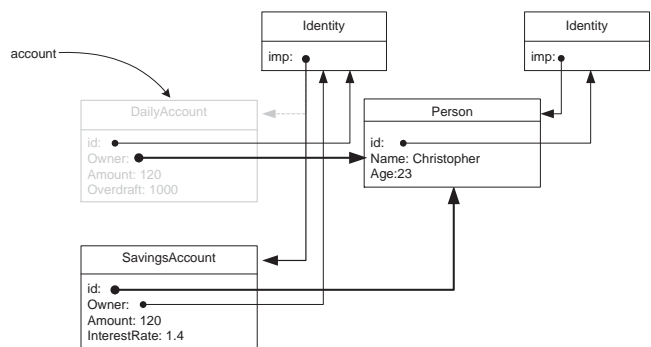

After Reclassification

Fig. 10: Objects in Version_3

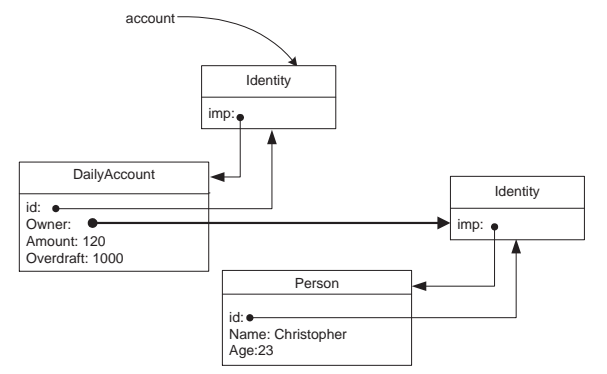

Before Re-classification

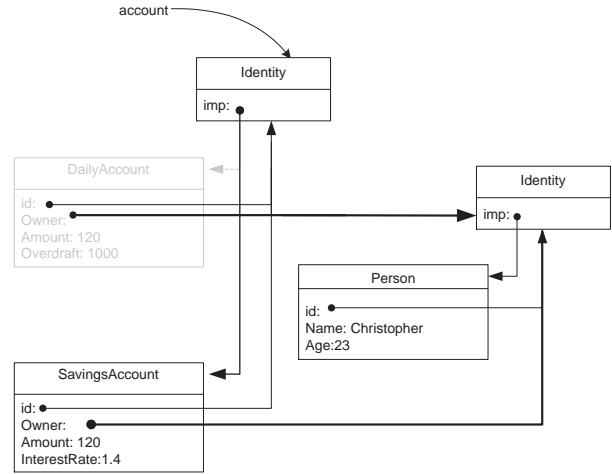

After Re-classification

Fig. 11: Objects in Version_4

\begin{tabular}{|l||c|c|c|c|}
\hline & Version_1 & Version_2 & Version_3 & Version_4 \\
\hline $\begin{array}{l}\text { Variables of type } c \text { are repre- } \\
\text { sented by variables of type: }\end{array}$ & $\mathcal{R}(p, c)$ & $\mathcal{R}(p, c)$ & $\mathcal{R}(p, c)$ & Identity \\
\hline $\begin{array}{l}\text { References represented } \\
\text { through reference to: }\end{array}$ & implementor & identity & implementor & identity \\
\hline The id field has type: & $\mathcal{R}(p, c)$ & Fickle0bject & Identity & Identity \\
\hline The imp field has type: & $\mathcal{R}(p, c)$ & FickleObject & FickleObject & FickleObject \\
\hline $\begin{array}{l}\text { Expression of type } c \text { trans- } \\
\text { lates to expression of type: }\end{array}$ & $\mathcal{R}(p, c)$ & $\mathcal{R}(p, c)$ & $c$ & Identity \\
\hline $\begin{array}{l}\text { References to outdated ob- } \\
\text { jects }\end{array}$ & Possible & Impossible & Possible & Impossible \\
\hline
\end{tabular}

Fig. 12: Comparison of translation approaches 


\begin{tabular}{|c|c|c|c|c|}
\hline$\overline{\mathcal{F}}$ ickle $_{\mathrm{II}}$ & Version_1 & Version_2 & Version_3 & Version_4 \\
\hline $\mathrm{A}$ a & $\mathrm{A}$ a & A a & $\mathrm{A} \quad \mathrm{a}$ & Identity a \\
\hline $\mathrm{Pp}$ & $\mathrm{Pp}$ & $P p$ & $\mathrm{Pp}$ & Identity $\mathrm{p}$ \\
\hline a.i & a.i & ((A)a.imp).i & $((A)((A) a \cdot i d \cdot i m p)) \cdot i$ & ((A)a.imp).i \\
\hline this.o & $\begin{array}{l}\text { ((SA)this } \\
\text {.id.imp).o }\end{array}$ & $\begin{array}{l}\text { ((SA)((A)this.id) } \\
\text {.imp).o }\end{array}$ & $\begin{array}{l}((\mathrm{P})((\mathrm{SA}) \text { this.id.imp })) \\
\text { o.id.imp) }\end{array}$ & $\begin{array}{l}\text { ((SA)this.id } \\
\text {.imp).o }\end{array}$ \\
\hline $\mathrm{a} 1=\mathrm{a} 2$ & $\mathrm{a} 1=\mathrm{a} 2$ & $\mathrm{a} 1=\mathrm{a} 2$ & $\mathrm{a} 1=(\mathrm{A})(\mathrm{a} 2$. id. imp $)$ & $a 1=a 2$ \\
\hline $\mathrm{SA}$ sa & -- & -- & -- & Identity sa \\
\hline sa.o & -- & -- & -- & sa.imp.o \\
\hline
\end{tabular}

Fig. 13: Differences between the translation of expressions, with Account, SavingsAccount, Person, Account : : interest, Account : : owner represented by $\mathrm{A}, \mathrm{SA}, \mathrm{P}, \mathrm{i}$, o respectively. a1,a2 have type Account and this has type SavingsAccount.

\section{Isabella, a prototype implementation}

Our prototype implementation, Isabella, follows Version_4 to map $\mathcal{F}_{-}$ckle st $^{\text {st }}$ onto Java. It is an extension of Carmela [4], which followed Version_1. Isabella is written in Java and follows a design based on the current Sun Java compiler (version 1.4). Isabellaconsists of a type checker and code generator, both implemented using the visitor pattern [14] as in the Java compiler. The whole compiler consists of approximately 6000 lines of code and can be found at WEBREF. Isabella extends $\mathcal{F}$ ickle ${ }^{\text {st }}$ in order to make testing easier, the extensions include:

- Output via System. out

- Integers and booleans with relevant operations such as ++,-- etc.

One of the challenges in Isabella was representing the blocks of code that arise in the translation Version_4. Noting that Java does not allow blocks as expressions, any entity in the source that requires a block of code in the translation must be represented as a flattened statement and a fresh local variable to contain the result. The variable can then be used where the value of the block is required.

For example, consider the translation of $a=$ new DailyAccount (); a.transact ( 1200 ); a.interest () ; For expression, a = new DailyAccount () we first translate new DailyAccount to get:

DailyAccount s1;

Identity $\mathrm{s} 2$;

$\mathrm{s} 2=$ new Identity () ;

s1= new DailyAccount ();

s1.id = s2;

s2.imp = s1;

Note the use of new temporary variables s1 and s2, with the result being in variable s2. The assignment to variable $\mathrm{a}$ is represented as a $=\mathrm{s} 2$. For method call a.transact (1200) we have: 


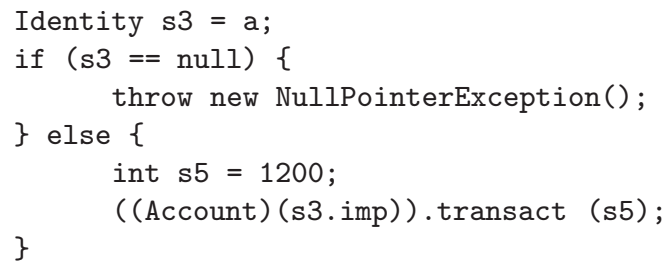

Note the use of temporary variables $\mathrm{s} 3$ for the receiver and (assuming no NullPointerException) s5 for the argument. As method transact is void we have no temporary variable for the return value. Finally for method call a.interest(); we have:

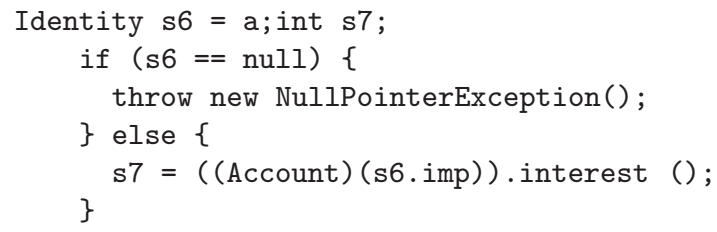

As with the previous method call we have a temporary variable for the receiver and in this case a temporary variable for the result $\mathrm{s} 7$.

Thus, the code produced by Isabella is identical to that produced by the translation given in this paper, apart from erasure of blocks and the extra features mentioned above.

\section{Conclusions and Further Work}

We have defined a translation from $\mathcal{F}_{\text {ickle }}$ II into $\mathcal{F}_{i c k l e}-\overline{\text { II }}$ (the subset of $\mathcal{F}$ ickle II $_{\text {II }}$ without re-classification), and have proven that this translation is well-behaved in the sense that it preserves static and dynamic semantics. The translation described in this paper is the basis of Isabella, an implementation which maps $\mathcal{F} i c k l e^{\text {st }}$ onto Java.

The proofs give us confidence in the correctness of Isabella, but they also gave us guidance in the design of the translation. Namely, we needed to define a correspondence between $\mathcal{F} i c k l e_{\text {II }}$ objects and $\mathcal{F} i c k l e_{\text {II }}^{-}$objects, and also between $\mathcal{F} i c k l e_{\text {II }}$ heaps and $\mathcal{F}$ ickle $e_{\text {II }}^{-}$heaps. These correspondences were necessary for the formulation of the theorems, but also expressed concepts that arose naturally as properties of the translation. We had already defined these concepts informally at the earlier versions, and in the later versions, their complete and formal definition guided the design of the translation of $\mathcal{F} i c k l e_{\text {II }}$ class definitions and $\mathcal{F} i c k l l_{\text {II }}$ expressions.

This work started when we decided to implement $\mathcal{F}$ ickle, through a translation into a Java. We expected the translation to be straightforward, and were surprised how many subtle points needed consideration, and how many alternative designs were possible. In fact, we now realize that the formalization of the translation and the formulation of the correspondences were indispensable. We conclude that any such translation tasks should not be attempted before a prior formalization of the languages, the translation and its invariants.

Except for the prototype implementations, we are interested in investigating the possibility of implementing an extension of Java with re-classification. From this point 
of view, our translation is a good basis since it exhibits the following additional properties:

- It is fully compatible with Java separate compilation, since each $\mathcal{F}$ ickle II $_{\text {c }}$ class can be translated without having other class bodies. Therefore, it would be sufficient to have the other classes in binary form, as done by current Java compilers.

- The dependencies across classes are exactly those of standard Java compilation, in the sense that a $\mathcal{F} i c k l e_{\text {II }}$ class can be translated only if the type information from all the ancestor and all used classes is available.

Further work includes the extension of $\mathcal{F}$ ickle II onto the full Java language. On the one hand, such an extension should take into account other Java features (like overloading) which, though in principle orthogonal to re-classification, should be carefully analyzed in order to be sure that the interaction behaves correctly. On the other hand, as mentioned above, an extended compiler should be able to work even in a context where only binary files are available, while our prototype implementation works on source files. 


\section{References}

1. D. Ancona, C. Anderson, F. Damiani, S. Drossopoulou, P. Giannini, and E. Zucca. An effective translation of Fickle into Java (extended abstract). In ICTCS'01, LNCS 2202, pages 215-234. Springer, 2001.

2. D. Ancona, C. Anderson, F. Damiani, S. Drossopoulou, P. Giannini, and E. Zucca. A type preserving translation of Fickle into Java. In TOSCA'01, volume 62 of ENTCS. Elsevier, 2002.

3. D. Ancona, G. Lagorio, and E. Zucca. Jam - a smooth extension of Java with mixins. In ECOOP'00, volume 1850 of LNCS, pages 154-178. Springer, 2000.

4. Christopher Anderson. Implementing Fickle, Imperial College, final year thesis to appear, June 2001.

5. Christopher Anderson. Isabella-Fickle translator, December 2003. Avaliable at http://www.macs.hw.ac.uk/DART/software/isabella/index.html.

6. C. Chambers. Predicate Classes. In ECOOP'93, volume 707 of LNCS, pages 268-296. Springer, 1993.

7. F. Damiani, M. Dezani-Ciancaglini, S. Drossopoulou, and P. Giannini. Refined effects for re-classification: FickleIII. DART report, part of Deliverable D3.1, available at the url http://www.cee.hw.ac.uk/DART/, December 2002.

8. F. Damiani, S. Drossopoulou, and P. Giannini. Refined effects for unanticipated object re-classification: Fickle3 (extended abstract). In ICTCS'03, LNCS 2841, pages 97-110. Springer, 2003.

9. Ferruccio Damiani, Mariangiola Dezani-Ciancaglini, and Paola Giannini. Reclassification and multithreading. In OOPS track at SAC'04. ACM, 2004. To appear.

10. S. Drossopoulou, F. Damiani, M. Dezani-Ciancaglini, and P. Giannini. Fickle: Dynamic object re-classification. In Electronic proceedings of FOOL8 (http://www.cs.williams.edu/kim/FOOL/), 2001.

11. S. Drossopoulou, F. Damiani, M. Dezani-Ciancaglini, and P. Giannini. Fickle: Dynamic object re-classification. In ECOOP'01, LNCS. Springer, 2001. To appear. A preliminary version is available in: Electronic proceedings of FOOL8 (http://www.cs.williams.edu/ kim/FOOL/).

12. S. Drossopoulou, F. Damiani, M. Dezani-Ciancaglini, and P. Giannini. More dynamic object re-classification: Fickle ${ }_{I I}$. Transactions On Programming Languages and Systems, 24(2):153-191, 2002.

13. M. D. Ernst, C. Kaplan, and C. Chambers. Predicate Dispatching: A Unified Theory of Dispatch. In ECOOP'98, volume 1445 of LNCS, pages 186-211. Springer, 1998.

14. Erich Gamma, Richard Helm, Ralph Johnson, and John Vlissides. Design Patterns: Elements od Reusable Object-Oriented Software. Addison-Wesley Professional Computing Series. Addison-Wesley Publishing Company, New York, NY, 1995.

15. W.L. Hürsch. Should Superclasses be Abstract? In ECOOP'94, volume 821 of LNCS, pages 12-31. Springer, 1994.

16. B. C. Pierce. Types and Programming Languages. The MIT Press, Cambridge, MA, 2002.

17. M. Serrano. Wide Classes. In ECOOP'99, volume 1628 of LNCS, pages 391-415. Springer, 1999. 


\section{A Operational semantics, definitions of lookup, subtypes, acyclic programs, and agreements}

Figures 14, 15, 16, and 17 list all the rewrite rules of $\mathcal{F} i c k l e_{\mathrm{II}}$.

Fig. 18 defines the judgment $\vdash p \diamond_{u}$, which guarantees that a program has unique definitions. In the judgment defs is defined by

$$
\text { defs }::=(\text { field } \mid \text { meth })^{*}
$$

The first requirement says that there should be no more than one class definition for any identifier $c$ - note that it implicitly guarantees $c^{\prime}=c^{\prime \prime}$ and that the class bodies are identical. The second requirement says that there should be no more than one field definition in $c$ for any identifier $f$-note that it implicitly guarantees $t=t^{\prime}$. The third requirement says that there should be a unique method definition in $c$ for any identifier $m$ - note that it implicitly guarantees $t=t^{\prime}, t_{1}=t_{1}^{\prime}, \ldots, t_{q}=t_{q^{\prime}}, x_{1}=x_{1}^{\prime}, \ldots, x_{q}=x_{q^{\prime}}$, $\phi=\phi^{\prime}$, and block $=$ block' .

For program $p$ with $\vdash p \diamond_{u}$, class name $c \neq$ Object, and qualifier qual = root, or $q u a l=$ state, or qual $=\epsilon$, we define the lookup of the class declaration for $c$ :

$\mathcal{C}(p, c)= \begin{cases}q u a l \text { class } c \text { extends } c^{\prime}\{\text { defs }\} & \text { if } p=p^{\prime} \text { qual class } c \text { extends } c^{\prime}\{c B o d y\} p^{\prime \prime} \\ \mathcal{U} d f & \text { otherwise }\end{cases}$

The assertion $p \vdash c \sqsubseteq c^{\prime}$, defined in Fig. 19, means that the class $c$ is a subclass of $c^{\prime}$. The class hierarchy in a program $p$ is well-formed, i.e., $\vdash p \diamond_{h}$, if the subclass relationship is acyclic, root classes extend only non-root and non-state classes, and state classes extend either root classes or state classes. Notice that $\vdash p \diamond_{u}$ whenever $\vdash p \diamond_{h}$.

It is straightforward to prove the following properties of programs with well-formed inheritance hierarchies: Two types that are in the subclass relationship are classes, the relation $\sqsubseteq$ is reflexive, transitive and antisymmetric, and the subclass hierarchy forms a tree with Object at its root.

The following judgments, also defined in Fig. 19, distinguish the kinds of classes: $p \vdash c \diamond_{c t}$ means that $c$ is any class, $p \vdash c \diamond_{r t}$ means that $c$ is a re-classifiable type i.e., either a root or a state class. The judgment $p \vdash t \diamond_{f t}$ means that $t$ is a non-state type, i.e., either a primitive type or a non-state class.

Widening, the extension of the subclass relationship to types, is expressed by the assertion $p \vdash t \leq t^{\prime}$, and is also defined by the rules in Fig. 19.

Environment lookup and update, and the least upper bound operation on types and environments are defined in Fig. 20.

For program $p$ with $\vdash p \diamond_{h}$, class name $c$ such that

$$
\mathcal{C}(p, c)=[\text { root } \mid \text { state }] \text { class } c \text { extends } c^{\prime}\{\text { defs }\}
$$

field name $f$ and method name $m$ we define: 


$$
\begin{aligned}
& \overline{s v, \sigma \underset{p}{\sim} s v, \sigma} \quad(v a l) \\
& e, \sigma \underset{p}{\longrightarrow} \iota, \sigma^{\prime} \\
& \sigma^{\prime}(\iota)=[[\ldots]]^{c^{\prime}}
\end{aligned}
$$

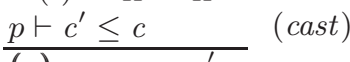

$$
\begin{aligned}
& (c) e, \sigma \underset{p}{\longrightarrow} \iota, \sigma^{\prime} \\
& \sigma(i d) \neq \mathcal{U} d f \\
& \stackrel{i d, \sigma \underset{p}{\longrightarrow} \sigma(i d), \sigma}{(i d)} \\
& e, \sigma \underset{p}{\longrightarrow} \operatorname{null}, \sigma^{\prime} \\
& \frac{i \operatorname{isnull}(e), \sigma \underset{p}{ } \text { true, } \sigma^{\prime}}{(t-i s n u l l)}
\end{aligned}
$$

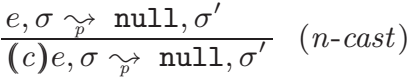

$$
\begin{aligned}
& e, \sigma \underset{p}{\longrightarrow} \iota, \sigma^{\prime} \\
& \sigma^{\prime}(\iota)=[[\ldots]]^{c^{\prime}} \\
& \frac{p \nvdash c^{\prime} \leq c}{(c) e, \sigma \leadsto \operatorname{castExc}, \sigma^{\prime}} \quad(e \text {-cast }) \\
& \sigma^{\prime}(\iota)(f) \neq \mathcal{U} d f
\end{aligned}
$$

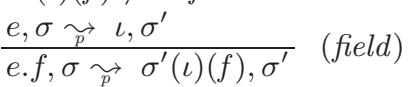

$$
\begin{aligned}
& e, \sigma \underset{p}{\longrightarrow} \iota, \sigma^{\prime} \\
& \sigma^{\prime}(\iota) \neq \mathcal{U} d f \\
& \frac{\text { isnull }(e), \sigma \underset{p}{\longrightarrow} \text { false, } \sigma^{\prime}}{(f-i s n u l l)} \\
& e, \sigma \underset{p}{\longrightarrow} \iota, \sigma^{\prime \prime} \\
& e_{1}, \sigma^{\prime \prime} \underset{p}{\longrightarrow} v, \sigma^{\prime \prime \prime} \\
& (a-v a r)
\end{aligned}
$$

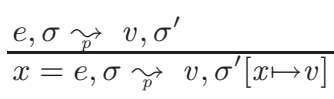

$$
\begin{aligned}
& \sigma^{\prime \prime \prime}(\iota)(f) \neq \mathcal{U} d f \\
& \frac{\sigma^{\prime}=\sigma^{\prime \prime \prime}\left[\iota \mapsto \sigma^{\prime \prime \prime}(\iota)[f \mapsto v]\right]}{e \cdot f=e_{1}, \sigma \underset{p}{\sim} v, \sigma^{\prime}} \\
& e, \sigma \underset{p}{\longrightarrow} \text { true, } \sigma^{\prime \prime} \\
& \frac{e_{1}, \sigma^{\prime \prime} \underset{p}{ }{ }^{\prime \prime} v, \sigma^{\prime}}{\text { if } e \text { then } e_{1} \text { else } e_{2}, \sigma \underset{p}{\longrightarrow} v, \sigma^{\prime}} \\
& (t \text {-cond }) \\
& e, \sigma \underset{p}{\longrightarrow} \text { false, } \sigma^{\prime \prime} \\
& e_{2}, \sigma^{\prime \prime} \underset{p}{\sim} v, \sigma^{\prime} \\
& \text { if } e \text { then } e_{1} \text { else } e_{2}, \sigma \underset{p}{\sim} v, \sigma^{\prime} \\
& \text { (a-field) } \\
& v_{l} \text { initial for } t_{l}(l \in\{1, \ldots, s\}) \\
& \sigma_{0}=\sigma\left[x_{1} \mapsto v_{1}, \ldots, x_{s} \mapsto v_{s}\right] \\
& \frac{e_{i}, \sigma_{i-1} \underset{p}{\longrightarrow} v_{i}, \sigma_{i}(i \in\{1, \ldots, n\})}{\left\{t_{1} x_{1} ; t_{s} x_{s} ; e_{1} ; \ldots e_{n}\right\}, \sigma v_{n}} \\
& \left\{t_{1} x_{1} ; \ldots t_{s} x_{s} ; e_{1} ; \ldots e_{n}\right\}, \sigma \underset{p}{\longrightarrow} v_{n}, \sigma_{n}\left[x_{1} \mapsto \sigma\left(x_{1}\right), \ldots, x_{s} \mapsto \sigma\left(x_{s}\right)\right]
\end{aligned}
$$

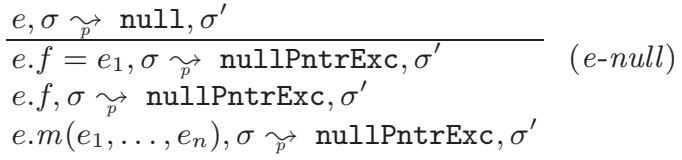

Fig. 14: Fickle $_{\text {II }}$ expression evaluation - without generation and propagation of errors 


$$
\begin{aligned}
& \sigma(i d)=\iota \\
& \sigma(\iota)=[[\ldots]]^{c} \\
& \mathcal{F}_{s}(p, \mathcal{R}(p, c))=\left\{f_{1}, \ldots, f_{r}\right\} \\
& v_{l}=\sigma(\iota)\left(f_{l}\right) \quad(l \in\{1, \ldots, r\}) \\
& \mathcal{F} s(p, d) \backslash\left\{f_{1}, \ldots, f_{r}\right\}=\left\{f_{r+1}, \ldots, f_{r+q}\right\} \\
& (\mathrm{recl}) \quad(n-\mathrm{recl}) \\
& v_{l} \text { initial for } \mathcal{F}\left(p, d, f_{l}\right) \quad(l \in\{r+1, \ldots, r+q\}) \quad \sigma(i d)=\text { null }
\end{aligned}
$$

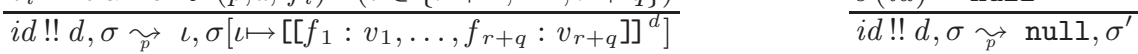

Fig. 15: $\mathcal{F}_{\text {ickle }}$ II expression evaluation - without generation and propagation of errors

$$
\begin{aligned}
& \mathcal{F D}(p, c, f) \quad= \begin{cases}t & \text { if defs }=\ldots t f \ldots \\
\mathcal{U} d f & \text { otherwise }\end{cases} \\
& \mathcal{F}(p, c, f)= \begin{cases}\mathcal{F} \mathcal{D}(p, c, f) & \text { if } \mathcal{F} \mathcal{D}(p, c, f) \neq \mathcal{U} d f, \\
\mathcal{F}\left(p, c^{\prime}, f\right) & \text { otherwise }\end{cases} \\
& \mathcal{F}(p, \text { object }, f)=\mathcal{U} d f \\
& \mathcal{F} s(p, c) \quad=\quad\{f \mid \mathcal{F}(p, c, f) \neq \mathcal{U} d f\} \\
& \mathcal{M D}(p, c, m)= \begin{cases}t m\left(t_{1} x_{1}, \ldots, t_{n} x_{n}\right) \phi \text { body } & \text { if defs }=\ldots t m\left(t_{1} x_{1} \ldots t_{n} x_{n}\right) \phi \text { body } \ldots \\
\mathcal{U} d f & \text { otherwise }\end{cases} \\
& \mathcal{M}(p, c, m) \quad= \begin{cases}\mathcal{M D}(p, c, m) & \text { if } \mathcal{M D}(p, c, m) \neq \mathcal{U} d f, \\
\mathcal{M}\left(p, c^{\prime}, m\right) & \text { otherwise }\end{cases} \\
& \mathcal{M}(p, \text { object }, m)=\mathcal{U} d f
\end{aligned}
$$

\section{B Proof of preservation of static semantics}

We write $\gamma \subseteq \gamma^{\prime}$ if for all id $\gamma(i d) \neq \mathcal{U} d f \Rightarrow \gamma(i d)=\gamma^{\prime}(i d)$.

Lemma 1. Let $p$ be a program, $\gamma, \gamma^{\prime}$ two environments s.t. $\gamma \subseteq \gamma^{\prime}$, e an expression, and $t$ a type.

If $p, \gamma \vdash e: t \rrbracket \gamma \rrbracket\{\}$, then $p, \gamma^{\prime} \vdash e: t \rrbracket \gamma^{\prime} \llbracket\{\}$.

Proof. First, by induction on the typing rules for expressions the following claim can be proved:

(*) if $p, \gamma \vdash e: t \rrbracket \gamma^{\prime} \llbracket\{\}$, then $\gamma=\gamma^{\prime}$.

Then, the lemma is proved by induction on the typing rules using claim $(*)$.

Lemma 2. Let $p$ be a program, $\gamma, \gamma^{\prime}$ two environments, $e$ an expression, $t$ a type, and $\phi$ an effect.

If $p, \gamma \vdash e: t \llbracket \gamma^{\prime} \llbracket \phi$ is provable, then $\llbracket \gamma \rrbracket=\llbracket \gamma^{\prime} \rrbracket$.

Proof. By induction on the typing rules for expressions.

Lemma 3. Let $p$ be a program s.t. $\vdash p \diamond, c, c^{\prime}$ two class names s.t. $p \vdash c \leq c^{\prime}, f a$ field name, $m$ a method name, $t, t_{1}, \ldots, t_{n}$ types $(n \geq 0), x_{1}, \ldots, x_{n}$ variables, and $\phi$ an effect. Then 


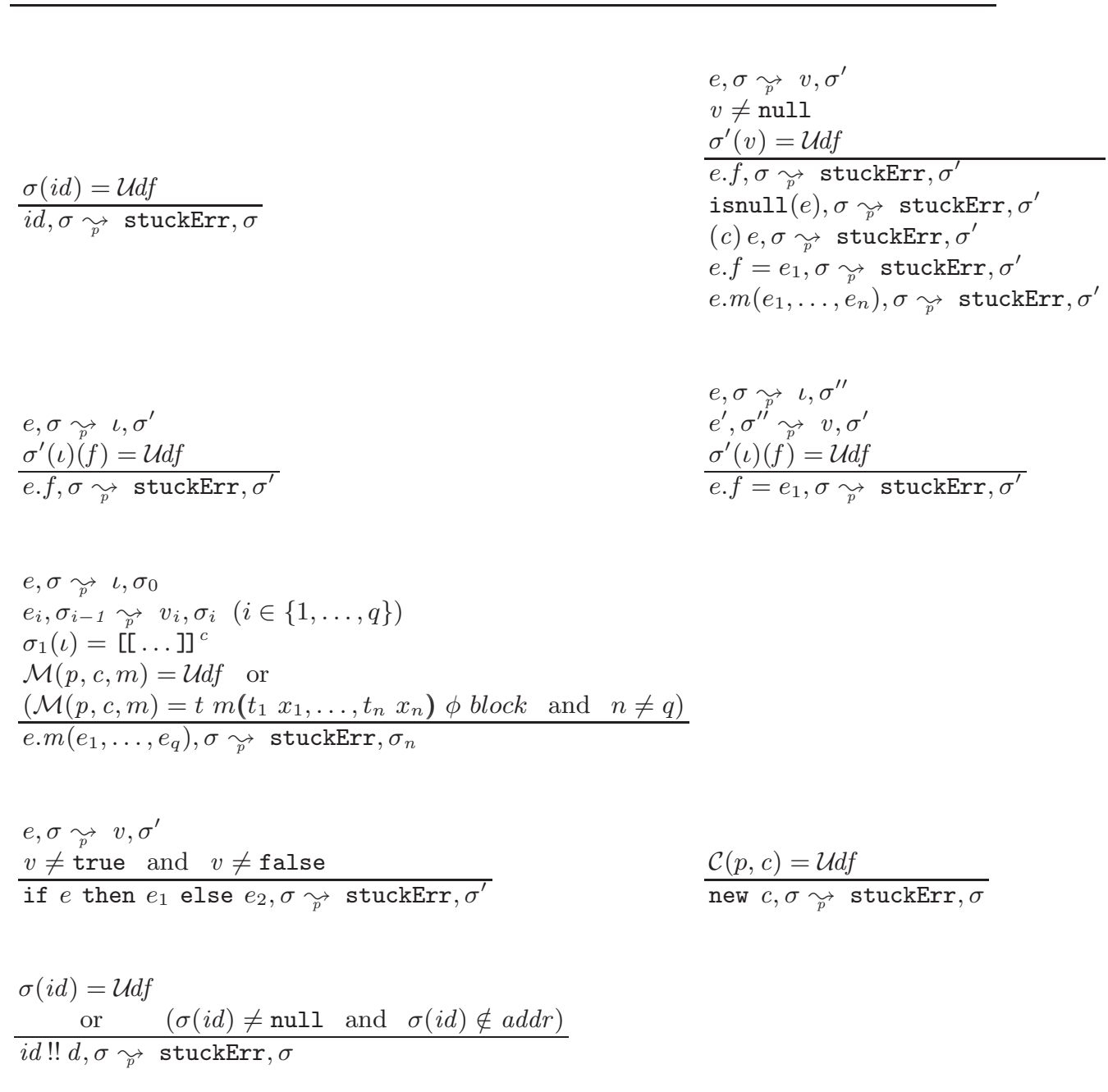

Fig. 16: $\mathcal{F}_{i c k l e}$ II expression evaluation - generation of errors 


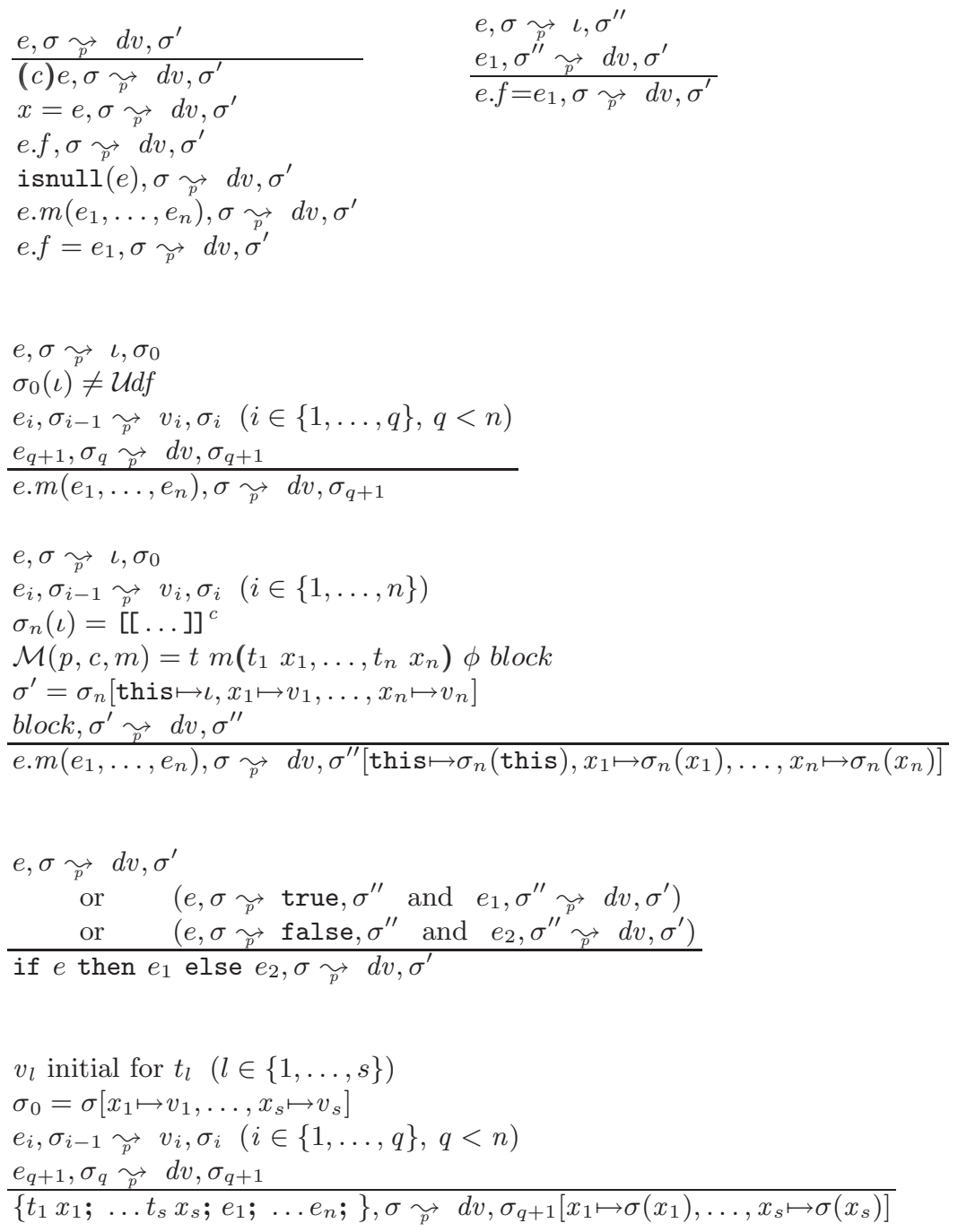

Fig. 17: Fickle $_{\text {II }}$ expression evaluation - propagation of exceptions and errors 


$$
\begin{aligned}
& \forall c: \quad p=p_{1}\left[\text { root } \mid \text { state] class } c \text { extends } c^{\prime}\{\ldots\} p_{2},\right. \\
& p=p_{3}[\text { root } \mid \text { state }] \text { class } c \text { extends } c^{\prime \prime}\{\ldots\} p_{4} \\
& \Longrightarrow p_{1}=p_{3}, p_{2}=p_{4} \\
& \forall f: \quad p=p_{1}\left[\text { root } \mid \text { state] class } c \text { extends } c^{\prime}\left\{\operatorname{defs}_{1} t f d e f s_{2}\right\} p_{2}\right. \text {, } \\
& p=p_{1}[\text { root } \mid \text { state }] \text { class } c \text { extends } c^{\prime}\left\{\text { defs }_{3} t^{\prime} f \text { defs }_{4}\right\} p_{2} \\
& \Longrightarrow \text { defs }_{1}=\text { defs }_{3} \text {, defs } s_{2}=\text { defs }_{4} \text {; } \\
& \forall m: p=p_{1} \text { [root | state] class } c \text { extends } c^{\prime}\left\{\operatorname{defs}_{1} t m\left(t_{1} x_{1}, \ldots, t_{q} x_{q}\right) \phi \text { block defs } s_{2}\right\} p_{2}, \\
& p=p_{1}[\text { root } \mid \text { state }] \text { class } c \text { extends } c^{\prime}\left\{\operatorname{defs}_{3} t^{\prime} m\left(t_{1}^{\prime} x_{1}^{\prime} \ldots, t_{n}^{\prime} x_{n}^{\prime}\right) \phi^{\prime} \text { block }^{\prime} \text { defs }_{4}\right\} p_{2} \\
& \Longrightarrow \text { defs }_{1}=\operatorname{defs}_{3}, \operatorname{defs}_{2}=\operatorname{defs}_{4}
\end{aligned}
$$

Fig. 18: Programs with unique definitions

$$
\begin{aligned}
& \vdash p \diamond_{u} \quad \quad p \vdash c \sqsubseteq c^{\prime}
\end{aligned}
$$

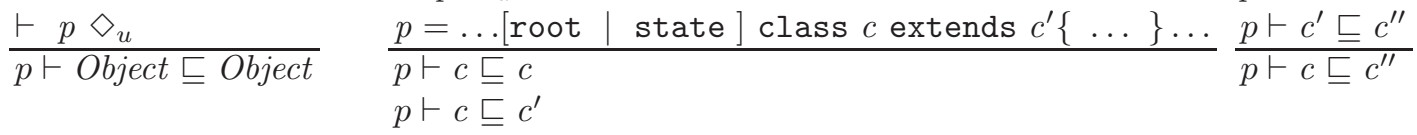

$$
\begin{aligned}
& \forall c, c^{\prime} \text { : } \\
& p \vdash c \sqsubseteq c^{\prime} \text { and } p \vdash c^{\prime} \sqsubseteq c \quad \Longrightarrow \quad c=c^{\prime} \\
& \mathcal{C}(p, c)=\text { class } c \text { extends } c^{\prime}\{\ldots\} \quad \Longrightarrow \quad \mathcal{C}\left(p, c^{\prime}\right)=\text { class } c^{\prime} \ldots \\
& \mathcal{C}(p, c)=\text { root class } c \text { extends } c^{\prime}\{\ldots\} \quad \Longrightarrow \quad \mathcal{C}\left(p, c^{\prime}\right)=\text { class } c^{\prime} \ldots \\
& \mathcal{C}(p, c)=\text { state class } c \text { extends } c^{\prime}\{\ldots\} \quad \Longrightarrow \\
& \left(\left(\mathcal{C}\left(p, c^{\prime}\right)=\text { root class } c^{\prime} \ldots\right) \text { or }\left(\mathcal{C}\left(p, c^{\prime}\right)=\text { state class } c^{\prime} \ldots\right)\right) \\
& \vdash p \diamond_{h} \\
& \vdash p \diamond_{h} \quad \vdash p \diamond_{h} \quad \vdash p \diamond_{h} \\
& \frac{\mathcal{C}(p, c)=\operatorname{class} c \ldots}{p \vdash c \diamond_{f t}} \quad \frac{\mathcal{C}(p, c)=\operatorname{root} \text { class } c \ldots}{p \vdash c \diamond_{f t}} \quad \frac{\mathcal{C}(p, c)=\text { state class } c \ldots}{p \vdash c \diamond_{r t}} \\
& p \vdash c \diamond_{c t} \quad p \vdash c \diamond_{r t} \quad p \vdash c \diamond_{c t} \\
& p \vdash c \diamond_{c t} \\
& \overline{p \vdash \text { bool } \diamond_{f t}} \quad \frac{p \vdash c \sqsubseteq c^{\prime}}{p \vdash \text { bool } \leq \text { bool }}
\end{aligned}
$$

Fig. 19: Subclasses, well-formed inheritance hierarchy, subtypes 


$$
\begin{gathered}
\gamma=x_{1}: t_{1}, \ldots, x_{n}: t_{n}, \text { this }: c \\
\gamma(\text { id })=\left\{\begin{array}{ll}
t_{i} \text { if } \text { id }=x_{i} \\
c \quad \text { if } \text { id }=\text { this } \\
\mathcal{U} d f \text { otherwise }
\end{array} \quad \gamma[\text { id } \mapsto t]\left(\text { id }^{\prime}\right)=\left\{\begin{array}{l}
t \quad \text { if } \text { id }^{\prime}=\text { id } \\
\gamma\left(\text { id }^{\prime}\right) \text { otherwise }
\end{array}\right.\right. \\
t_{1} \sqcup_{p} t_{2}= \begin{cases}t \quad \text { if } p \vdash t_{1} \leq t & p \vdash t_{2} \leq t \quad \forall t^{\prime} \cdot\left(p \vdash t_{1} \leq t^{\prime} \text { and } p \vdash t_{2} \leq t^{\prime}\right) \Rightarrow p \vdash t \leq t^{\prime} \\
\mathcal{U} d f \text { otherwise }\end{cases} \\
\gamma \sqcup_{p} \gamma^{\prime}=\left\{i d:\left(t \sqcup_{p} t^{\prime}\right) \mid \gamma(i d)=t \text { and } \gamma^{\prime}(i d)=t^{\prime}\right\}
\end{gathered}
$$

Fig. 20: Environment lookup and update, lub on types and environments

1. $\mathcal{F}\left(p, c^{\prime}, f\right)=t \Rightarrow \mathcal{F}(p, c, f)=t$

2. $\mathcal{M}\left(p, c^{\prime}, m\right)=t m\left(t_{1} x_{1}, \ldots, t_{n} x_{n}\right) \phi\{\ldots\} \Rightarrow$

$\mathcal{M}(p, c, m)=t m\left(t_{1} x_{1}, \ldots, t_{n} x_{n}\right) \phi^{\prime}\{\ldots\}$ for some effect $\phi^{\prime}$.

Proof. By induction on the height of the inheritance tree.

Lemma 4. Let $p$ be a program s.t. $\vdash p \diamond$, c a class name, $f$ a field name, $m$ a method name, $t, t_{1}, \ldots, t_{n}$ types $(n \geq 0), x_{1}, \ldots, x_{n}$ variables, and $\phi$ an effect. Then

1. $\mathcal{F}(p, c, f)=\mathcal{U} d f \Rightarrow \mathcal{F}\left(\llbracket p \rrbracket_{\text {prog }}\right.$, theName $\left.(c), f\right)=\mathcal{U} d f$;

2. $\mathcal{F}(p, c, f)=t \Rightarrow \mathcal{F}\left(\llbracket p \rrbracket_{\text {prog }}\right.$, theName $\left.(c), f\right)=$ theType $(t)$;

3. $\mathcal{M}(p, c, m)=\mathcal{U} d f \Rightarrow \mathcal{M}\left(\llbracket p \rrbracket_{\text {prog }}\right.$, theName $\left.(c), m\right)=\mathcal{U} d f$;

4. $\mathcal{M}(p, c, m)=t m\left(t_{1} x_{1}, \ldots, t_{n} x_{n}\right) \phi\{\ldots\} \Rightarrow \mathcal{M}\left(\llbracket p \rrbracket_{\text {prog }}\right.$, theName $\left.(c), m\right)=$ theType $(t) m$ (theType $\left(t_{1}\right) x_{1}, \ldots$, theType $\left.\left(t_{n}\right) x_{n}\right)\{\}\{\ldots\}$.

Proof. By induction on the height of the inheritance tree.

Lemma 5. Let $p$ be a program s.t. $\vdash p \diamond$, and $c$ a class name s.t. $p \vdash c \diamond_{c t}$. Then $\mathcal{F}\left(\llbracket p \rrbracket_{\text {prog }}\right.$, the Name $(c)$, id $)=$ Identity.

Proof. By induction on the height of the inheritance tree.

Lemma 6. Let $p$ be a program s.t. $\vdash p \diamond$, and $c$ a class name s.t. $p \vdash c \diamond_{c t}$. Then $\llbracket p \rrbracket_{\text {prog }} \vdash$ theName $(c) \leq$ FickleObject.

Proof. By induction on the height of the inheritance tree.

Lemma 7. Let $p$ be a program.

If $\vdash p \diamond$, then $\vdash \llbracket p \rrbracket_{\text {prog }} \diamond_{h}$.

Proof. By induction on the height of the inheritance tree.

Lemma 8. Let $p$ be a program, and $t, t^{\prime}$ two types.

Then $p \vdash t \leq t^{\prime}$ implies $\llbracket p \rrbracket_{\text {prog }} \vdash$ the Type $(t) \leq$ the Type $\left(t^{\prime}\right)$. 
Proof. Trivial.

Lemma 9. Let $p$ be a program s.t. $\vdash p \diamond$. Then $\llbracket p \rrbracket_{\text {prog }} \vdash$ Identity $\diamond_{c t}$.

Proof. Trivial.

Lemma 10. Let $p$ be a program s.t. $\vdash p \diamond$. Then

$\mathcal{F}\left(\llbracket p \rrbracket_{\text {prog }}\right.$, Identity, imp $)=$ FickleObject.

Proof. Trivial.

Lemma 11. Let $p$ be a program s.t. $\vdash p \diamond, t$ a type, and $\phi$ an effect.

Then $p \vdash t \leq \phi @_{p} t$.

Proof. Trivial.

Lemma 12. Let $p$ be a program s.t. $\vdash p \diamond$, and $t, t^{\prime}$ two types.

Then the Type $\left(t \sqcup_{p} t^{\prime}\right)=$ the Type $(t) \sqcup_{\llbracket p \rrbracket_{\text {prog }}}$ the Type $\left(t^{\prime}\right)$.

Proof. Trivial.

\section{Proof of Theorem 2}

Proof. The proof proceeds by induction on the typing rules (or, equivalently, on the structure of expressions) and by case analysis on the kinds of expressions and relies on the fact that the generation (or inversion) lemma for the typing relation, see [16], is trivial since there is a one-to-one relation between typing rules and kinds of expressions.

Cases $e \equiv$ true, false, $n$ are trivial.

Case $e \equiv i d$.

If $i d \equiv x$, then $\llbracket e \rrbracket_{\text {expr }}(p, \gamma) \equiv x$ and by hypothesis $\gamma(x)=t$, therefore $\llbracket \gamma \rrbracket(x)=$ the Type $(t)$ and we can conclude by applying the suitable typing rule.

If $i d \equiv$ this, then $\llbracket e \rrbracket_{\operatorname{expr}}(p, \gamma) \equiv$ this.id and by hypothesis $\gamma($ this $)=t$, with $p \vdash t \diamond_{c t}$ and $t \neq 0$ bject; therefore theType $(t)=$ Identity, theName $(t)=t, \llbracket \gamma \rrbracket($ this $)=t$, and Lemma 5 is applicable hence $\mathcal{F}\left(\llbracket p \rrbracket_{\text {prog }}, t\right.$, id $)=$ Identity; we conclude by applying the suitable typing rules.

Case $e \equiv$ null.

Then $\llbracket e \rrbracket_{\text {expr }}(p, \gamma) \equiv$ null and by hypothesis $p \vdash t \diamond_{c t}$, therefore theType $(t)=$ Identity and by Lemma $9 \llbracket p \rrbracket_{\text {prog }} \vdash$ Identity $\diamond_{c t}$, hence we can conclude by applying the suitable typing rule.

Case $e \equiv \operatorname{isnull}\left(e^{\prime}\right)$.

Then $\llbracket e \rrbracket_{e x p r}(p, \gamma) \equiv \operatorname{isnull}\left(\llbracket e^{\prime} \rrbracket_{\operatorname{expr}}(p, \gamma)\right)$ and by hypothesis $p, \gamma \vdash e^{\prime}: c \rrbracket \gamma^{\prime} \llbracket \phi$, therefore by inductive hypothesis $\llbracket p \rrbracket_{\text {prog }}, \llbracket \gamma \rrbracket \vdash \llbracket e^{\prime} \rrbracket_{\text {expr }}(p, \gamma)$ : Identity $\llbracket \llbracket \gamma \rrbracket \llbracket\{\}$, hence we can conclude by applying the suitable typing rule.

Case $e \equiv$ new $c$.

Then $\llbracket e \rrbracket_{\text {expr }}(p, \gamma) \equiv\{$ theName $(c)$ theImp;

Identity theId;

theId = new Identity;

theImp = new theName $(c)$;

theImp.id = theId;

theId.imp = theImp;

theId 
where theImp and theId are chosen s.t. $\gamma($ theImp $)=\mathcal{U} d f, \gamma($ theId $)=\mathcal{U} d f$. By hypothesis $p \vdash t \diamond_{c t}$, therefore theType $(t)=$ Identity; furthermore by Lemmas 5,10 and 6 it is possible to apply the suitable typing rules to derive the conclusion.

Case $e \equiv(c) e^{\prime}$.

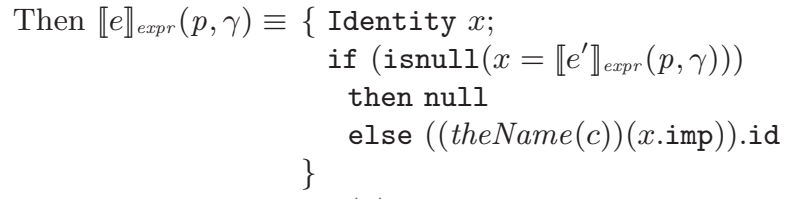

where $x$ is chosen s.t. $\gamma(x)=\mathcal{U} d f$. By hypothesis $p, \gamma \vdash e^{\prime}: c^{\prime}\left\|\gamma^{\prime}\right\| \phi$ and $t=c$, therefore theType $(t)=$ Identity and by inductive hypothesis

$\llbracket p \rrbracket_{\text {prog }}, \llbracket \gamma \rrbracket \vdash \llbracket e^{\prime} \rrbracket_{\text {expr }}(p, \gamma)$ : Identity $\llbracket \llbracket \gamma \rrbracket \llbracket\{\}$ and by Lemma 1 ,

$\llbracket p \rrbracket_{p r o g}, \llbracket \gamma_{0} \rrbracket \vdash \llbracket e^{\prime} \rrbracket_{\text {expr }}(p, \gamma):$ Identity $\llbracket \llbracket \gamma_{0} \rrbracket \rrbracket\{\}$ where $\gamma_{0}=\llbracket \gamma \rrbracket[x \mapsto$ Identity $]$; finally, by Lemmas $5,9,10$ and 6 , it is possible to apply the suitable typing rules in order to conclude.

Case $e \equiv e^{\prime} . f$.

By hypothesis $p, \gamma \vdash e^{\prime}: c \rrbracket \gamma^{\prime} \llbracket \phi$, then the translation is well-defined:

$\llbracket e \rrbracket_{\text {expr }}(p, \gamma) \equiv\left((\right.$ theName $(c))\left(\llbracket e^{\prime} \rrbracket_{\text {expr }}(p, \gamma) \cdot\right.$ imp $\left.)\right) . f$

By inductive hypothesis $\llbracket p \rrbracket_{\text {prog }}, \llbracket \gamma \rrbracket \vdash \llbracket e^{\prime} \rrbracket_{\text {expr }}(p, \gamma)$ : Identity $\llbracket \llbracket \gamma \rrbracket \rrbracket\{\}$, furthermore, by hypothesis $\mathcal{F}(p, c, f)=t$, therefore by Lemma $4, \mathcal{F}\left(\llbracket p \rrbracket_{\text {prog }}\right.$, theName $\left.(c), f\right)=$ the Type $(t)$; finally, by Lemmas 10 and 6 , it is possible to apply the suitable typing rules in order to conclude.

Case $e \equiv e_{1} \cdot f=e_{2}$.

By hypothesis $p, \gamma \vdash e_{1}: c \rrbracket \gamma_{1} \| \phi_{1}$ and $p, \gamma_{1} \vdash e_{2}: t \llbracket \gamma_{2} \rrbracket \phi_{2}$, then the translation is well-defined:

$\llbracket e \rrbracket_{\text {expr }}(p, \gamma) \equiv\left\{\right.$ Identity $x_{1}$

the Type $(t) x_{2}$

if $\left(\right.$ isnull $\left.\left(x_{1}=\llbracket e_{1} \rrbracket_{\text {expr }}(p, \gamma)\right)\right)$

then null. $f=x_{2}$

else $\left\{x_{2}=\llbracket e_{2} \rrbracket_{\text {expr }}\left(p, \gamma_{1}\right)\right.$;

\} \}

$\left(\left(\phi_{2} @_{p}\right.\right.$ theName $\left.(c)\right)\left(x_{1}\right.$.imp $\left.)\right) . f=x_{2}$

with $x_{1}$ and $x_{2}$ chosen s.t. $\gamma\left(x_{1}\right)=\gamma\left(x_{2}\right)=\mathcal{U} d f$. By inductive hypothesis

$\llbracket p \rrbracket_{\text {prog }}, \llbracket \gamma \rrbracket \vdash \llbracket e_{1} \rrbracket_{\text {expr }}(p, \gamma)$ : Identity $\rrbracket \llbracket \gamma \rrbracket \llbracket\{\}$ and

$\llbracket p \rrbracket_{\text {prog }}, \llbracket \gamma_{1} \rrbracket \vdash \llbracket e_{2} \rrbracket_{\text {expr }}\left(p, \gamma_{1}\right):$ theType $(t) \rrbracket \llbracket \gamma_{1} \rrbracket \llbracket\{\}$

furthermore by Lemma $2, \llbracket \gamma \rrbracket=\llbracket \gamma_{1} \rrbracket$, and by Lemma 1 ,

$\llbracket p \rrbracket_{\text {prog }}, \gamma_{0} \vdash \llbracket e_{1} \rrbracket_{\text {expr }}(p, \gamma):$ Identity $\llbracket \gamma_{0} \rrbracket\{\}$ and

$\llbracket p \rrbracket_{\text {prog }}, \gamma_{0} \vdash \llbracket e_{2} \rrbracket_{\text {expr }}\left(p, \gamma_{1}\right):$ theType $(t) \rrbracket \gamma_{0} \rrbracket\{\}$

with $\gamma_{0}=\llbracket \gamma \rrbracket\left[x_{1} \mapsto\right.$ Identity, $x_{2} \mapsto$ theType $\left.(t)\right]$.

Again, by hypothesis $\mathcal{F}\left(p, \phi_{2} @_{p} c, f\right)=t^{\prime}$ and $p \vdash t \leq t^{\prime}$, and by definition theName $\left(\phi_{2} @_{p} c\right)=$ $\phi_{2} @_{p}$ theName $(c)$, therefore by Lemmas 4 and 8, $\mathcal{F}\left(\llbracket p \rrbracket_{\text {prog }}, \phi_{2} @_{p}\right.$ theName $\left.(c), f\right)=$ the Type $\left(t^{\prime}\right)$ and $\llbracket p \rrbracket_{\text {prog }} \vdash$ the Type $(t) \leq$ theType $\left(t^{\prime}\right)$. Finally, by Lemmas 10 and 6 , it is possible to apply the suitable typing rules in order to conclude.

Case $e \equiv x=e_{1}$.

Then $\llbracket e \rrbracket_{\text {expr }}(p, \gamma) \equiv x=\llbracket e_{1} \rrbracket_{e x p r}(p, \gamma)$

By hypothesis $p, \gamma \vdash e_{1}: t \rrbracket \gamma^{\prime} \rrbracket \phi, \gamma^{\prime}(x)=t^{\prime}$ and $p \vdash t \leq t^{\prime}$, therefore by inductive hypothesis and Lemmas 2 and $8, \llbracket p \rrbracket_{\text {prog }}, \llbracket \gamma \rrbracket \vdash \llbracket e_{1} \rrbracket_{\operatorname{expr}}(p, \gamma):$ theType $(t) \rrbracket \llbracket \gamma \rrbracket \llbracket\{\}$, 
$\llbracket \gamma \rrbracket=\llbracket \gamma^{\prime} \rrbracket$ hence $\llbracket \gamma \rrbracket(x)=$ theType $\left(t^{\prime}\right)$, and $\vdash$ theType $(t) \leq$ theType $\left(t^{\prime}\right)$. Finally, it is possible to apply the suitable typing rule in order to conclude.

Case $e \equiv e_{0} \cdot m\left(e_{1}, \ldots, e_{n}\right)$.

By hypothesis, $p, \gamma \vdash e_{0}: c\left\|\gamma_{0}\right\| \phi_{0}$ and $p, \gamma_{i-1} \vdash e_{i}: t_{i}\left\|\gamma_{i}\right\| \phi_{i}$ for all $i=1, \ldots, n$, then the translation is well-defined:

$\llbracket e \rrbracket_{\text {expr }}(p, \gamma) \equiv\{$ Identity $x$

the Type $\left(t_{1}\right) x_{1}$;

$\ldots$

the Type $\left(t_{n}\right) x_{n}$;

if $\left(\operatorname{isnull}\left(x=\llbracket e_{0} \rrbracket_{\text {expr }}(p, \gamma)\right)\right)$

then null.m $\left(x_{1}, \ldots, x_{n}\right)$

else $\left\{x_{1}=\llbracket e_{1} \rrbracket_{\text {expr }}\left(p, \gamma_{0}\right)\right.$

$\cdots$

$x_{n}=\llbracket e_{n} \rrbracket_{\operatorname{expr}}\left(p, \gamma_{n-1}\right)$

$\left(\left(\left(\phi_{1} \cup \cdots \cup \phi_{n}\right) @_{p}\right.\right.$ theName $\left.\left.(c)\right)(x . \mathrm{imp})\right) . m\left(x_{1}, \ldots, x_{n}\right)$

\}

\}

with $x, x_{1}, \ldots, x_{n}$ chosen s.t. $\gamma(x)=\gamma\left(x_{1}\right)=\cdots=\gamma\left(x_{n}\right)=\mathcal{U} d f$. By Lemma 2, $\llbracket \gamma \rrbracket=\llbracket \gamma_{0} \rrbracket=\cdots=\llbracket \gamma_{n} \rrbracket$, and by inductive hypothesis and Lemma 1 ,

$\llbracket p \rrbracket_{\text {prog }}, \gamma^{\prime} \vdash \llbracket e_{0} \rrbracket_{\text {expr }}(p, \gamma):$ Identity $\llbracket \gamma^{\prime} \rrbracket\{\}$ and

$\llbracket p \rrbracket_{\text {prog }}, \gamma^{\prime} \vdash \llbracket e_{i} \rrbracket_{\text {expr }}\left(p, \gamma_{i-1}\right):$ theType $\left(t_{i}\right) \rrbracket \gamma^{\prime} \rrbracket\{\}$ for all $i=1, \ldots, n$, with $\gamma^{\prime}=\llbracket \gamma \rrbracket\left[x \mapsto\right.$ Identity, $x_{1} \mapsto$ theType $\left(t_{1}\right), \ldots, x_{n} \mapsto$ the Type $\left.\left(t_{n}\right)\right]$.

Again by hypothesis, $\mathcal{M}\left(p,\left(\phi_{1} \cup \cdots \cup \phi_{n}\right) @_{p} c, m\right)=t m\left(t_{1}^{\prime} x_{1}, \ldots, t_{n}^{\prime} x_{n}\right) \phi\{\ldots\}$ and $p \vdash\left(\phi_{i+1} \cup \cdots \cup \phi_{n}\right) @_{p} t_{i} \leq t_{i}^{\prime}$ for all $i=1, \ldots, n$, and by definition theName $\left(\left(\phi_{1} \cup \cdots \cup \phi_{n}\right) @_{p} c\right)=$

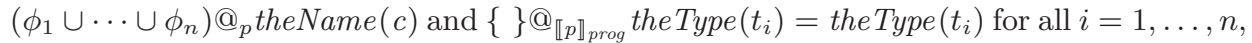
therefore by Lemmas 4,8 and 11 and transitivity of the subtyping relation,

$\mathcal{M}\left(\llbracket p \rrbracket_{\text {prog }},\left(\phi_{1} \cup \cdots \cup \phi_{n}\right) @_{p} \operatorname{theName}(c), m\right)=$

the Type $(t) m$ (theType $\left(t_{1}^{\prime}\right) x_{1}, \ldots$, theType $\left.\left(t_{n}^{\prime}\right) x_{n}\right)\{\}\{\ldots\}$ and

$\llbracket p \rrbracket_{\text {prog }} \vdash\{\} @ \llbracket p \rrbracket_{\text {prog }}$ the Type $\left(t_{i}\right) \leq$ theType $\left(t_{i}^{\prime}\right)$ for all $i=1, \ldots, n$. Finally, by Lemmas 10

and 6 it is possible to apply the suitable typing rules in order to conclude.

Case $e \equiv i d ! ! c$.

Let us consider the case $i d \equiv x$ (the case $i d \equiv$ this is analogous); the translation is defined by

$\llbracket e \rrbracket_{e x p r}(p, \gamma) \equiv\{$ Identity theId;

theName (c) theImp;

$\mathcal{R}(p, c)$ theLastImp;

if $($ isnull $($ theId $=x))$

then null

else $\{$ theImp = new theName $(c)$;

theLastImp $=(\mathcal{R}(p, c))$ (theId.imp);

theId.imp = theImp;

theImp.id = theId;

theImp. $f_{1}=$ theLastImp. $f_{1}$;

...

\};

theImp. $f_{r}=$ theLastImp. $f_{r}$

theId

\}

with theId, theImp and theLastImp chosen s.t. $\gamma($ theImp $)=\gamma($ theId $)=\gamma($ theLastImp $)=$ $\mathcal{U} d f$ and $\left\{f_{1}, \ldots, f_{r}\right\}=\mathcal{F} s(p, \mathcal{R}(p, c))$. 
By hypothesis, $p \vdash c \diamond_{r t}, \mathcal{R}(p, c)=\mathcal{R}(p, \gamma(x))$. By definition, $f_{i} \in \mathcal{F} s(p, \mathcal{R}(p, c)) \Rightarrow$ $\exists t \mathcal{F}\left(p, \mathcal{R}(p, c), f_{i}\right)=t$ for all $i=1, \ldots, r$; furthermore, since by definition $p \vdash$ $c \leq \mathcal{R}(p, c)$, by Lemma $3, \mathcal{F}\left(p, c, f_{i}\right)=t$ for all $i=1, \ldots, r$. Since by definition, theName $(\mathcal{R}(p, c))=\mathcal{R}(p, c)$, by Lemma $4, \mathcal{F}\left(\llbracket p \rrbracket_{\text {prog }}, \mathcal{R}(p, c), f_{i}\right)=\mathcal{F}\left(\llbracket p \rrbracket_{\text {prog }}\right.$, theName $\left.(c), f_{i}\right)=$ the Type $(t)$ for all $i=1, \ldots, r$. Finally, by Lemmas 5, 9, 10 and 6 the suitable typing rules can be applied in order to conclude.

Case $e \equiv$ if $e_{0}$ then $e_{1}$ else $e_{2}$.

By hypothesis,

$p, \gamma \vdash e_{0}:$ bool $\llbracket \gamma_{0} \llbracket \phi_{0}$

$p, \gamma_{0} \vdash e_{1}: t_{1} \llbracket \gamma_{1} \llbracket \phi_{1}$

$p, \gamma_{0} \vdash e_{2}: t_{2} \llbracket \gamma_{2} \llbracket \phi_{2}$

Then the translation is well-defined:

$\llbracket e \rrbracket_{\text {expr }}(p, \gamma) \equiv$ if $\llbracket e_{0} \rrbracket_{\text {expr }}(p, \gamma)$ then $\llbracket e_{1} \rrbracket_{e x p r}\left(p, \gamma_{0}\right)$ else $\llbracket e_{2} \rrbracket_{\text {expr }}\left(p, \gamma_{0}\right)$

By Lemma 2, $\llbracket \gamma \rrbracket=\llbracket \gamma_{0} \rrbracket=\llbracket \gamma_{1} \rrbracket=\llbracket \gamma_{2} \rrbracket$ and by inductive hypothesis,

$\llbracket p \rrbracket_{\text {prog }}, \llbracket \gamma \rrbracket \vdash \llbracket e_{0} \rrbracket_{\text {expr }}(p, \gamma):$ bool $\llbracket \llbracket \gamma \rrbracket \llbracket\{\}$

$\llbracket p \rrbracket_{\text {prog }}, \llbracket \gamma \rrbracket \vdash \llbracket e_{1} \rrbracket_{\text {expr }}\left(p, \gamma_{0}\right): \operatorname{theType}\left(t_{1}\right) \llbracket \llbracket \gamma \rrbracket \llbracket\{\}$

$\llbracket p \rrbracket_{\text {prog }}, \llbracket \gamma \rrbracket \vdash \llbracket e_{2} \rrbracket_{\text {expr }}\left(p, \gamma_{0}\right): \operatorname{theType}\left(t_{2}\right) \llbracket \llbracket \gamma \rrbracket \llbracket\{\}$

Finally, it is possible to conclude by applying the suitable typing rule and Lemma 12.

Case $e \equiv\left\{t_{1} x_{1} ; \ldots t_{s} x_{s} ; e_{1} ; \ldots e_{n}\right\}$.

By hypothesis,

$\gamma_{0}=\gamma\left[x_{1} \mapsto t_{1}, \ldots, x_{s} \mapsto t_{s}\right]$

$p, \gamma_{i-1} \vdash e_{i}: t_{i}^{\prime} \rrbracket \gamma_{i} \rrbracket \phi_{i}$ for all $i=1, \ldots, n$

Then the translation is well-defined:

$\llbracket e \rrbracket_{\text {expr }}(p, \gamma) \equiv\left\{\llbracket t_{1} x_{1} \rrbracket_{v a r} ; \ldots \llbracket t_{s} x_{s} \rrbracket_{v a r} ; \llbracket e_{1} \rrbracket_{\text {expr }}\left(p, \gamma_{0}\right) ; \ldots \llbracket e_{n} \rrbracket_{e x p r}\left(p, \gamma_{n-1}\right)\right\}$

By Lemma $2, \llbracket \gamma_{0} \rrbracket=\cdots \llbracket \gamma_{n} \rrbracket$ and by translation of variable declarations and by inductive hypothesis,

$\llbracket p \rrbracket_{\text {prog }}, \llbracket \gamma_{0} \rrbracket \vdash \llbracket e_{i} \rrbracket_{\text {expr }}\left(p, \gamma_{i-1}\right):$ theType $\left(t_{i}^{\prime}\right) \rrbracket \llbracket \gamma_{0} \rrbracket \llbracket\{\}$ for all $i=1, \ldots, n$

where, by definition, $\llbracket \gamma_{0} \rrbracket=\llbracket \gamma \rrbracket\left[x_{1} \mapsto\right.$ the Type $\left(t_{1}\right), \ldots, x_{s} \mapsto$ theType $\left.\left(t_{s}\right)\right]$. Finally, it is possible to conclude by applying the suitable typing rule.

\section{Proof of Theorem 3}

Proof. Let us take an arbitrary class named $c$ and defined in $p$ (recall that in the translation of programs - see comments in Section 5.1 - we assume no name conflicts, so that $c \neq$ Identity, FickleObject and $f \neq$ id for any field $f$ declared in $p$ ); by translation of class and field declarations, for any type $t_{f}^{\prime}$, and field name $f \neq i$ d, $\mathcal{F} \mathcal{D}\left(\llbracket p \rrbracket_{\text {prog }}, c, f\right)=t_{f}^{\prime}$ implies $\mathcal{F} \mathcal{D}(p, c, f)=t_{f}$, for a type $t_{f}$ s.t. the Type $\left(t_{f}\right)=t_{f}^{\prime}$. By definition of the Type, $\llbracket p \rrbracket_{\text {prog }} \vdash t_{f}^{\prime} \diamond_{f t}$; furthermore, by hypothesis, $\mathcal{F}\left(p, c^{\prime}, f\right)=\mathcal{U} d f$, therefore, by Lemma $4, \mathcal{F}\left(\llbracket p \rrbracket_{\text {prog }}\right.$, the Name $\left.\left(c^{\prime}\right), f\right)=\mathcal{U} d f$.

By translation of class and method declarations, for all types $t_{0}^{\prime}, \ldots, t_{n}^{\prime}(n \geq 0)$, method name $m$, variables $x_{1}, \ldots, x_{n}$, effect $\phi_{0}$, and block block',

$\mathcal{M D}\left(\llbracket p \rrbracket_{\text {prog }}, c, m\right)=t_{0}^{\prime} m\left(t_{1}^{\prime} x_{1}, \ldots, t_{n}^{\prime} x_{n}\right) \phi_{0}$ block ${ }^{\prime} \Rightarrow$ $\phi_{0}=\{\}$ and $\mathcal{M D}(p, c, m)=t m\left(t_{1} x_{1}, \ldots, t_{n} x_{n}\right) \phi$ block,

for types $t, t_{1}, \ldots, t_{n}$, s.t. the Type $(t)=t_{0}^{\prime}$, theType $\left(t_{i}\right)=t_{i}^{\prime}$ for $i=1, \ldots, n$, an effect $\phi$, and a block block s.t. $\llbracket b l o c k \rrbracket_{\text {expr }}(p, \gamma)=$ block', with $\gamma=t_{1} x_{1}, \ldots, t_{n} x_{n}, c$ this.

Therefore $p \vdash \phi_{0} \diamond$ trivially holds, $\llbracket p \rrbracket_{\text {prog }}, \llbracket \gamma \rrbracket \vdash$ block ${ }^{\prime}:$ theType $\left(t^{\prime}\right) \llbracket \llbracket \gamma \rrbracket \llbracket\{\}$ is provable by hypothesis and Theorem $2, \llbracket p \rrbracket_{\text {prog }} \vdash$ theType $\left(t^{\prime}\right) \leq t_{0}^{\prime}$ holds by hypothesis and Lemma 8 and trivially \{\}$\subseteq \phi_{0}$, therefore we can conclude by applying the typing rule for class declarations. 


\section{Proof of Theorem 4}

Proof. By Lemma $7, \vdash \llbracket p \rrbracket_{\text {prog }} \diamond_{h}$; by definition of Identity and FickleObject, and by the typing rules, $\llbracket p \rrbracket_{\text {prog }} \vdash$ Identity $\diamond$ and $\llbracket p \rrbracket_{\text {prog }} \vdash$ FickleObject $\diamond$. Moreover, every class $c \neq$ Identity, FickleObject defined in $\llbracket p \rrbracket_{\text {prog }}$ is defined also in $p$ (by definition of translation of programs and classes), and by Theorem 3 we have $\llbracket p \rrbracket_{\text {prog }} \vdash c \diamond$. Hence we can conclude the result from the typing rule for programs.

\section{Proof of preservation of dynamic semantics}

Lemma 13. Let $p$ be a program s.t. $\vdash p \diamond, c, c^{\prime}$ two class names. If $p \vdash c \leq c^{\prime}$, then $p \vdash$ theName $(c) \leq$ theName $\left(c^{\prime}\right)$.

Proof. If both $c$ and $c^{\prime}$ are different from Object then it is obvious since theName $(c)=c$ and theName $\left(c^{\prime}\right)=c^{\prime}$. If either one is equal to Object then since $p \vdash c \leq c^{\prime}$ it must be $c^{\prime}=$ Object. Therefore, theName $\left(c^{\prime}\right)=$ FickleObject and for all classes $d$ we have that $p \vdash d \leq$ FickleObject.

Lemma 14. Let $t$ be a type. If $v$ is the initial value of type $t$ then $v$ is also the initial value of type the Type $(t)$.

Proof. If $t$ is a class type then $v=$ null which is initial also for Identity, otherwise $t$ is a primitive type and theType $(t)=t$.

Lemma 15. Let e be an expression such that: $p, \gamma \vdash e: t \rrbracket \gamma^{\prime} \llbracket \phi$, and $\sigma$ be a store such that $\llbracket p \rrbracket, \llbracket \gamma \rrbracket[$ this $\mapsto c] \vdash \sigma \diamond$, for some $c$. If

$$
\llbracket e \rrbracket, \sigma \quad \widetilde{q p} \rrbracket \quad w, \sigma^{\prime}
$$

then for all $x, \gamma(x)=U d f$ implies $\sigma^{\prime}(x)=\sigma(x)$.

Proof. By induction on the depth of the derivation tree of $\llbracket e \rrbracket, \sigma \underset{\mathbb{p}}{ } \quad w, \sigma^{\prime}$. For the translation of the expressions corresponding to values, variables, this, null test, and field selection $\llbracket e \rrbracket$ does not contain assignments, therefore for all $x, \sigma(x)=\sigma^{\prime}(x)$.

For the translation of assignment, note that for $x=e^{\prime}$ to be well-typed, it must be $\gamma(x) \neq \mathcal{U} d f$. So since $\llbracket e \rrbracket \triangleq x=\llbracket e^{\prime} \rrbracket$, the result is by induction hypothesis on $\llbracket e^{\prime} \rrbracket$.

For all the other expressions note that the translation of the expression is a block in which the local variables $y$ are such that $\gamma(y)=\mathcal{U} d f$. From the operational semantics of blocks we have that for all the local variables $y, \sigma^{\prime}(y)=\sigma(y)$ (for all the local variables the value before execution of the block is restored). So the result is by induction hypotheses on the subexpressions.

\section{Proof of Theorem 5}

We will prove the two implications separately. 
Proof of: $e, \sigma \underset{\vec{p}}{\longrightarrow} w, \sigma^{\prime} \quad$ implies $\llbracket e \rrbracket, \sigma_{1} \underset{\widetilde{\| p} \rrbracket}{\sim} \boldsymbol{w}, \sigma_{1}^{\prime}$ By induction on the depth of the derivation tree of the judgement: $e, \sigma \underset{\vec{p}}{\longrightarrow} w, \sigma^{\prime}$. Note that, since $e$ is well-typed from Theorem 1 its evaluation cannot produce stuckErr so the result is either a value or $w \in\{$ castExc, nullPntrExc $\}$, and the rules that may have been used are the rules in Fig. 14, Fig. 15, and Fig. 17 where $d v \in\{$ castExc, nullPntrExc $\}$.

We will consider a subset of the rules applied. Namely: $(i d),($ new $),($ recl $),($ meth $)$, (cast), $(n$-cast $),(e-n u l l)$, and (e-cast). The other cases are similar, and for the propagation rules the result is derived directly from the inductive hypothesis.

Consider rule $(i d):$ id, $\sigma \underset{p}{\sim} \sigma(\mathrm{id}), \sigma$. Since $\llbracket \mathrm{id} \rrbracket_{\text {expr }}(p, \gamma) \triangleq$ id we derive that $\llbracket \mathrm{id} \rrbracket, \sigma_{1} \leadsto$ $\overline{\llbracket p \rrbracket} \sigma_{1}(\mathrm{id}), \sigma_{1}$. Moreover, $p, \gamma \vdash \sigma \approx \sigma_{1}$ implies that $\sigma(\mathrm{id})=\sigma_{1}(\mathrm{id})$.

Consider rule (new):

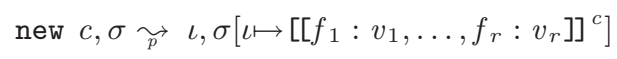

where $\iota$ is new in $\sigma$ and $v_{l}$ is initial for $\mathcal{F}\left(p, c, f_{l}\right), 1 \leq l \leq r$. Assume that $c \neq 0$ object, so theName $(c)=c$. (The proof for the case $c=$ Object is simpler since $\mathcal{F} s(p, c)=\emptyset$.) From the definition of the translation $\llbracket$ new $c \rrbracket(p, \gamma) \triangleq\left\{c\right.$ theImp; Identity theId; $e_{1} ; e_{2} ; e_{3} ; e_{4} ;$ theId $\}$ where

$-e_{1}$ is theId = new Identity,

$-e_{2}$ is the Imp $=$ new $c$,

$-e_{3}$ is theImp.id $=$ theId, and

$-e_{4}$ is theId.imp $=$ theImp.

and $\gamma($ theId $)=\gamma($ theImp $)=U d f$.

Let $\sigma_{1}^{\prime \prime}=\sigma_{1}[$ theImp $\mapsto$ null, theId $\mapsto$ null]. From the definition of the operational semantics of blocks, and the fact that null is the initial value for an object, we have that

$-e_{1}, \sigma_{1}^{\prime \prime} \widetilde{\llbracket p} \iota, \sigma_{2}$ where we assume that $\iota$ is new also in $\sigma_{1}$ (otherwise we could rename all its occurrences in $\sigma_{1}$ with a new address), and $\sigma_{2}=\sigma_{1}^{\prime \prime}\left[\iota \mapsto[\text { [imp : null] }]^{\text {Id }}\right.$, theId $\left.\mapsto \iota\right]$

$-e_{2}, \sigma_{2} \underset{\mathbb{p} \vec{\eta}}{ } \iota, \sigma_{3}$ where $\iota^{\prime} \neq \iota$ is new in $\sigma_{1}$ (we also assume that $\iota^{\prime}$ does not appear in $\sigma)$, and, since $c$ extends FickleObject, $\sigma_{3}=\sigma_{2}\left[\iota^{\prime} \mapsto\left[\left[\text { id }: \operatorname{null}, f_{1}: v_{1}^{\prime}, \ldots, f_{r}: v_{r}^{\prime}\right]\right]^{c}\right.$, theImp $\left.\mapsto \iota^{\prime}\right]$ and $v_{l}^{\prime}$ is initial for theType $\left(\mathcal{F}\left(p, c, f_{l}\right)\right), 1 \leq l \leq r$,

- $e_{3} ; e_{4}, \sigma_{3} \underset{\mathbb{p} \rrbracket}{\overleftrightarrow{p}} \iota, \sigma_{1}^{\prime \prime \prime}$, where $\sigma_{1}^{\prime \prime \prime}$ is $\sigma_{1}\left[\iota^{\prime} \mapsto\left[\left[\text { id }: \iota, f_{1}: v_{1}^{\prime}, \ldots, f_{r}: v_{r}^{\prime}\right]\right]^{c}\right.$, theImp $\mapsto \iota^{\prime}, \iota \mapsto\left[\left[\text { imp : } \iota^{\prime}\right]\right]^{\text {Id }}$, theId $\left.\mapsto \iota\right]$.

Let $\sigma_{1}^{\prime}=\sigma_{1}^{\prime \prime \prime}\left[\right.$ theImp $\mapsto \sigma_{1}($ theImp $)$, theId $\mapsto \sigma_{1}($ theId $\left.)\right]$

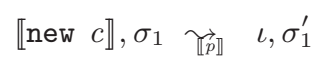

Let $\sigma^{\prime}=\sigma\left[\iota \mapsto\left[\left[f_{1}: v_{1}, \ldots, f_{r}: v_{r}\right]\right]^{c}\right]$. For all $x, \sigma^{\prime}(x)=\sigma(x)$, and $\sigma_{1}^{\prime}(x)=\sigma_{1}(x)$ (by definition of $\sigma_{1}^{\prime}$ ). Therefore, since $p, \gamma \vdash \sigma \approx \sigma_{1}$ clauses 1. and 2. of Definition 2 hold for $\sigma^{\prime}$ and $\sigma_{1}^{\prime}$. Moreover, for all $\iota^{\prime \prime}, \iota^{\prime \prime} \neq \iota, \sigma^{\prime}\left(\iota^{\prime \prime}\right)=\sigma\left(\iota^{\prime \prime}\right)$ and $\iota^{\prime \prime} \neq \iota^{\prime}$, implies also $\sigma_{1}^{\prime}\left(\iota^{\prime \prime}\right)=\sigma_{1}\left(\iota^{\prime \prime}\right)$. So we have to consider only $\iota$.

$-\sigma^{\prime}(\iota)=\left[\left[f_{1}: v_{1}, \ldots, f_{r}: v_{r}\right]\right]^{c}$,

$-\sigma_{1}^{\prime}(\iota)=\left[\left[\text { imp }: \iota^{\prime}\right]\right]^{\mathrm{Id}}, \sigma_{1}^{\prime}\left(\iota^{\prime}\right)=\left[\left[\text { id }: \iota, f_{1}: v_{1}^{\prime}, \ldots, f_{r}: v_{r}^{\prime}\right]\right]^{c}$ 
Since we assumed that $\sigma\left(\iota^{\prime}\right)=U d f$, and from Lemma 14, we derive that $v_{i}^{\prime}=v_{i}$, $1 \leq i \leq r$, then also clause 3. of Definition 2 is verified, and we conclude that $p, \overline{\gamma^{\prime}} \vdash \overline{\sigma^{\prime}} \approx \sigma_{1}^{\prime}$.

Consider rule $(\mathrm{recl})$, and assume that the expression is this !! $d$, therefore:

$$
\begin{aligned}
& \sigma(\text { this })=\iota \\
& \sigma(\iota)=[[\ldots]]^{c} \\
& \mathcal{F} s(p, \mathcal{R}(p, c))=\left\{f_{1}, \ldots, f_{r}\right\} \\
& v_{l}=\sigma(\iota)\left(f_{l}\right)(l \in\{1, \ldots, r\}) \\
& \mathcal{F} s(p, d) \backslash\left\{f_{1}, \ldots, f_{r}\right\}=\left\{f_{r+1}, \ldots, f_{r+q}\right\} \\
& v_{l} \text { initial for } \mathcal{F}\left(p, d, f_{l}\right) \quad(l \in\{r+1, \ldots, r+q\}) \\
& \hline \text { this } ! ! d, \sigma \widetilde{p} \iota, \sigma\left[\iota \longmapsto\left[\left[f_{1}: v_{1}, \ldots, f_{r+q}: v_{r+q}\right]\right]^{d}\right]
\end{aligned}
$$

Since the expression is well-typed, and Object is not a root or state class, we have that $d \neq 0 \mathrm{bject}$, and so theName $(d)=d$. From the definition of the translation we have:

$\llbracket$ this $! ! d \rrbracket(p, \gamma) \triangleq\left\{d\right.$ theImp; Identity theId; $\mathcal{R}(p, d)$ theLastThis; $e_{1} ; e_{2} ; e_{3} ; e_{4} ; e_{1}^{\prime} ; \cdots ; e_{r}^{\prime} ;$ theId $\}$

where

$-e_{1}$ is theId = this.id,

$-e_{2}$ is theLastThis $=(\mathcal{R}(p, d))$ theId.imp,

$-e_{3}$ is the Imp = new $d$,

$-e_{4}$ is theImp.id $=$ theId,

$-e_{5}$ is theId.imp $=$ the Imp

$-e_{i}^{\prime}$ is theImp. $f_{i}=$ theLastThis. $f_{i}$ for $1 \leq i \leq r$.

and $\gamma($ theId $)=\gamma($ theLastThis $)=\gamma($ theImp $)=U d f$. From the fact that $p, \gamma \vdash \sigma \approx \sigma_{1}$, and rule (1) we have that:

$$
\begin{aligned}
& \sigma_{1}(\text { this })=\iota^{\prime} \quad \sigma_{1}\left(\iota^{\prime}\right)=[[\text { id }: \iota, \ldots]]^{c^{\prime}} \quad \sigma_{1}(\iota)=\left[\left[\text { imp }: \iota^{\prime \prime}\right]\right]^{\text {Id }} \\
& \sigma_{1}\left(\iota^{\prime \prime}\right)=\left[\left[\text { id }: \iota, f_{1}: v_{1}, \ldots, f_{r}: v_{r}, f_{r+1}: v_{1}^{\prime}, \ldots, f_{r+p}: v_{p}^{\prime}\right]\right]^{c}
\end{aligned}
$$

and $v_{i}^{\prime}=\sigma$ (this $)\left(f_{r+i}\right)$ for $1 \leq i \leq p$.

Let $\sigma_{1}^{\prime \prime}=\sigma_{1}[$ theImp $\mapsto$ null, theId $\mapsto$ null, theLastThis $\mapsto$ null].From the definition of the operational semantics of blocks, the fact that null is the initial value for an object, we have that:

$-e_{1}, \sigma_{1}^{\prime \prime} \underset{\llbracket p}{\widetilde{p}} \quad \iota, \sigma_{1}^{\prime \prime \prime}$ where $\sigma_{1}^{\prime \prime \prime}=\sigma_{1}^{\prime \prime}[$ theId $\mapsto \iota]$

$-e_{2}, \sigma_{1}^{\prime \prime \prime} \underset{\llbracket p \rrbracket}{\longrightarrow} \iota^{\prime \prime}, \sigma_{2}$ where $\sigma_{2}=\sigma_{1}^{\prime \prime \prime}\left[\right.$ theLastThis $\left.\mapsto \iota^{\prime \prime}\right]$ since theId.imp, $\sigma_{1}^{\prime \prime \prime} \widetilde{\llbracket p \rrbracket}^{\prime \prime \prime}, \sigma_{1}^{\prime \prime \prime}$ and from the hypothesis that this!! $d$ is well-typed we have that $c \leq \mathcal{R}(p, d)$, so also $(\mathcal{R}(p, d))$ theId.imp, $\sigma_{1}^{\prime \prime \prime} \widetilde{\mathbb{p} p} \rrbracket \iota^{\prime \prime}, \sigma_{1}^{\prime \prime \prime}$ (no cast exception may occur).

$-e_{3}, \sigma_{2} \overrightarrow{\mathbb{p}} \rrbracket \quad \iota^{\prime \prime \prime}, \sigma_{3}$ where $\iota^{\prime \prime \prime}$ is new in $\sigma_{1}$ (we also assume that it does not appear in $\sigma)$, and, since $d$ extends FickleObject, $\sigma_{3}=\sigma_{2}\left[\iota^{\prime \prime \prime} \mapsto\left[\left[\text { id }: \operatorname{null}, f_{1}: v_{1}^{\prime \prime}, \ldots, f_{r}: v_{r}^{\prime \prime}, f_{r+1}: v_{r+1}^{\prime \prime}, \ldots, f_{r+q}: v_{r+q}^{\prime \prime}\right]\right]^{d}\right.$, theImp $\mapsto$ $\left.\iota^{\prime \prime \prime}\right]$ where $v_{l}^{\prime \prime}$ is initial for the Type $\left(\mathcal{F}\left(p, c, f_{l}\right)\right), l \in\{1, \ldots, r+q\}$,

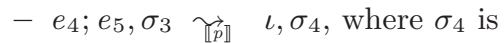
$\sigma_{3}\left[\iota \mapsto\left[\left[\text { imp }: \iota^{\prime \prime \prime}\right]\right]^{\mathrm{Id}}, \iota^{\prime \prime \prime} \mapsto\left[\left[\text { id }: \iota, f_{1}: v_{1}^{\prime \prime}, \ldots, f_{r}: v_{r}^{\prime \prime}, f_{r+1}: v_{r+1}^{\prime \prime}, \ldots, f_{r+q}: v_{r+q}^{\prime \prime}\right]\right]^{d}\right]$, and 


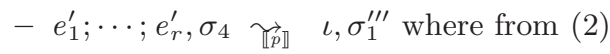

$\sigma_{1}^{\prime \prime \prime}=\sigma_{4}\left[\iota^{\prime \prime \prime} \mapsto\left[\left[\mathrm{id}: \iota, f_{1}: v_{1}, \ldots, f_{r}: v_{r}, f_{r+1}: v_{r+1}^{\prime \prime}, \ldots, f_{r+q}: v_{r+q}^{\prime \prime}\right]\right]^{d}\right]$.

Let $\sigma_{1}^{\prime}=\sigma_{1}^{\prime \prime \prime}\left[\right.$ theImp $\mapsto \sigma_{1}($ theImp $)$, theId $\mapsto \sigma_{1}($ theId $)$, theLastThis $\mapsto \sigma_{1}$ (theLastThis)].

$$
\llbracket \text { this !! } d \rrbracket, \sigma_{1} \text { 苑 } \rrbracket \quad \iota, \sigma_{1}^{\prime}
$$

Let $\sigma^{\prime}=\sigma\left[\iota \mapsto\left[\left[f_{1}: v_{1}, \ldots, f_{r+q}: v_{r+q}\right]^{c}\right]\right.$. We have to show that, $p, \gamma^{\prime} \vdash \sigma^{\prime} \approx \sigma_{1}^{\prime}$. First notice that from Theorem $1, p, \gamma^{\prime} \vdash \sigma^{\prime} \diamond$, and since $\llbracket p \rrbracket, \llbracket \gamma \rrbracket\left[\right.$ this $\left.\mapsto c^{\prime}\right] \vdash \sigma_{1} \diamond$, see $(2)$, also $\llbracket p \rrbracket, \llbracket \gamma^{\prime} \rrbracket\left[\right.$ this $\left.\mapsto c^{\prime}\right] \vdash \sigma_{1}^{\prime} \diamond$. So we have to show that the three clauses of Definition 2 are satisfied. From the definition $\sigma^{\prime}$ and $\sigma_{1}^{\prime}$, we have that for all $x$, $\sigma^{\prime}(x)=\sigma(x)$, and $\sigma_{1}^{\prime}(x)=\sigma_{1}(x)$. Therefore, clause 1. of Definition 2 holds for $\sigma^{\prime}$ and $\sigma_{1}^{\prime}$, and since $\sigma_{1}^{\prime}$ (this)(id) $=\sigma_{1}$ (this)(id), then also clause 2 . holds. To conclude the proof, we have to verify clause 3 . for the address $\iota$.

$-\sigma^{\prime}(\iota)=\left[\left[f_{1}: v_{1}, \ldots, f_{r+q}: v_{r+q}\right]\right]^{c}, \sigma_{1}^{\prime}(\iota)=\left[\left[\text { imp }: \iota^{\prime \prime}\right]\right]^{\text {Id }}$,

$-\sigma_{1}^{\prime}\left(\iota^{\prime \prime}\right)=\left[\left[\mathrm{id}: \iota, f_{1}: v_{1}, \ldots, f_{r}: v_{r}, f_{r+1}: v_{r+1}^{\prime \prime}, \ldots, f_{r+q}: v_{r+q}^{\prime \prime}\right]\right]^{d}$

where for $1 \leq i \leq q, v_{r+i}$ is an initial value for $\mathcal{F}\left(p, c, f_{r+i}\right)$ and $v_{r+i}^{\prime \prime}$ is an initial value for the Type $\left(\mathcal{F}\left(p, c, f_{r+i}\right)\right)$. Therefore, from Lemma 14, $v_{r+i}=v_{r+i}^{\prime \prime}$, and clause 3. holds. This concludes the proof that $p, \gamma^{\prime} \vdash \sigma^{\prime} \approx \sigma_{1}^{\prime}$.

The proof for the case in which the rule $(\mathrm{recl})$ is applied to $x$ !! $d$ is similar.

Consider rule $(n$-recl). In this case the expression must be $x$ !! $d$, since $p, \gamma \vdash \sigma \diamond$, see

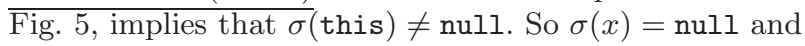

$$
x ! ! d, \sigma \quad \underset{\mathbb{p} \rrbracket}{\operatorname{null}}, \sigma .
$$

From the definition of the translation (as for the case $(r e c l)$, we have that the Name $(d)=$ d)

$$
\llbracket x ! ! d \rrbracket(p, \gamma) \triangleq\left\{d \text { theImp; Identity theId; } \mathcal{R}(p, d) \text { theLastImp; if } e_{1} \text { then } e_{2} ; \text { theId }\right\}
$$

where

$-e_{1}$ is $(($ theId $=x) \neq$ null $)$,

$-e_{2}$ is

$$
\begin{aligned}
& \text { \{theImp }=\text { new } d ; \text { theLastImp }=(\mathcal{R}(p, d)) \text { theId.imp; } \\
& \text { theImp.id }=\text { theId; theId.imp }=\text { theImp; } \\
& e_{1}^{\prime} ; \ldots e_{r}^{\prime} \\
& \}
\end{aligned}
$$

where $e_{i}^{\prime}$ is theImp. $f_{i}=$ theLastImp. $f_{i}$ for $1 \leq i \leq r$.

and $\gamma($ theId $)=\gamma($ theLastImp $)=\gamma($ theImp $)=U d f$.

Let $\sigma_{1}^{\prime \prime}=\sigma_{1}[$ theImp $\mapsto$ null, theId $\mapsto$ null, theLastImp $\mapsto$ null]. Observe that, from $p, \gamma \vdash \sigma \approx \sigma_{1}$ we derive that $\sigma_{1}(x)=$ null, and so also $\sigma_{1}^{\prime \prime}(x)=$ null. From the definition of the operational semantics of blocks, and the fact that null is the initial value for an object, we have that:

$$
e_{1}, \sigma_{1}^{\prime \prime} \underset{\llbracket p \rrbracket}{ } \text { false, } \sigma_{1}^{\prime \prime}
$$

Therefore, 
- if $e_{1}$ then $e_{2} ;$ theId, $\sigma_{1}^{\prime \prime} \widetilde{\llbracket p} \rrbracket$ null, $\sigma_{1}^{\prime \prime}$, and

$-\llbracket x ! ! d \rrbracket, \sigma_{1} \quad \widetilde{\natural p} \rrbracket \quad$ null, $\sigma_{1}$ since

$\sigma_{1}^{\prime}=\sigma_{1}^{\prime \prime}\left[\right.$ theImp $\mapsto \sigma_{1}($ theImp $)$, theId $\mapsto \sigma_{1}($ theId $)$, theLastImp $\mapsto \sigma_{1}($ theLastImp $\left.)\right]=$ $\sigma_{1}$, implies $\sigma_{1}^{\prime}=\sigma_{1}$.

Consider rule (meth): We will consider methods with a single parameter, e.m $\left(e^{\prime}\right)$. So

$$
\begin{aligned}
& \alpha . e, \sigma \underset{p}{\longrightarrow} \iota, \sigma^{\prime \prime} \\
& \beta . e^{\prime}, \sigma^{\prime \prime} \underset{p}{p} v^{\prime}, \sigma^{\prime \prime \prime} \\
& \chi \cdot \sigma^{\prime \prime \prime}(\iota)=[[\cdots]]^{c^{\prime}} \quad \mathcal{M D}\left(p, c^{\prime}, m\right)=t m\left(t_{1} y_{1}\right) \phi \text { block } \\
& \alpha^{\prime} . \bar{\sigma}=\sigma^{\prime \prime \prime}\left[\text { this } \mapsto \iota, y_{1} \mapsto v^{\prime}\right] \\
& \beta^{\prime} \text {. block, } \bar{\sigma} \underset{p}{\longrightarrow} v, \bar{\sigma}^{\prime} \\
& \chi^{\prime} . \sigma^{\prime}=\bar{\sigma}^{\prime}\left[\text { this } \mapsto \sigma^{\prime \prime \prime} \text { (this), } y_{1} \mapsto \sigma^{\prime \prime \prime}\left(y_{1}\right)\right] \\
& \text { e.m(e } \left.e^{\prime}\right), \sigma \underset{p}{\sim} v, \sigma^{\prime}
\end{aligned}
$$

From the fact that the expression is well-typed, we have

$$
\begin{aligned}
& \text { a. } p, \gamma \vdash e: c \rrbracket \gamma_{0} \llbracket \phi_{0} \\
& \text { b. } p, \gamma_{0} \vdash e^{\prime}: t_{1} \llbracket \gamma_{1} \llbracket \phi_{1} \\
& \text { c. } \mathcal{M D}\left(p, \phi_{1} @_{p} c, m\right)=t m\left(t_{1}^{\prime} y_{1}\right) \phi^{\prime} \text { block } \\
& \text { d. } p \vdash t_{1} \leq t_{1}^{\prime} \quad \phi=\phi_{0} \cup \phi_{1} \cup \phi^{\prime} \quad \gamma^{\prime}=\phi^{\prime} @_{p} \gamma_{1} \\
& \hline p, \gamma \vdash e \cdot m\left(e^{\prime}\right): t \rrbracket \gamma^{\prime} \rrbracket \phi
\end{aligned}
$$

Note that, since $\mathcal{M D}\left(p, \phi_{1} @_{p} c, m\right) \neq \mathcal{U} d f$, and $p \vdash c \leq \phi_{1} @_{p} c$, we have that $c \neq$ Object. Therefore theName $(c)=c$. Consider the translation of the expression, from (4) we have

$$
\llbracket e . m\left(e^{\prime}\right) \rrbracket_{\operatorname{expr}}(p, \gamma) \triangleq\left\{\text { Identity } x ; \text { theType }\left(t_{1}\right) x_{1} ; \text { if } e_{1} \text { then } e_{2} \text { else }\left\{e_{3} ; e_{4}\right\}\right\}
$$

where

$$
\begin{aligned}
& -e_{1} \text { is } \operatorname{isnull}\left(x=\llbracket e \rrbracket_{\text {expr }}(p, \gamma)\right) \\
& -e_{2} \text { is null.m }\left(x_{1}\right) \\
& -e_{3} \text { is } x_{1}=\llbracket e^{\prime} \rrbracket_{\text {expr }}\left(p, \gamma_{0}\right) \\
& -e_{4} \text { is }\left(\left(\phi_{1} @_{p} c\right)(x . \operatorname{imp})\right) \cdot m\left(x_{1}\right)
\end{aligned}
$$

and $\gamma\left(x_{1}\right)=\gamma(x)=\mathcal{U} d f$. Since $\gamma, \gamma_{0}$ and $\gamma^{\prime}$ are defined on the same set of identifiers, we have that also $\gamma_{0}\left(x_{1}\right)=\gamma_{0}(x)=\mathcal{U} d f$ and $\gamma^{\prime}\left(x_{1}\right)=\gamma^{\prime}(x)=\mathcal{U} d f$.

Let $\sigma_{1}^{\prime \prime}=\sigma_{1}\left[x \mapsto \operatorname{null}, x_{1} \mapsto v^{\prime}\right]$, where $v^{\prime}$ is an initial value of type the Type $(t)$. From $p, \gamma \vdash \sigma \approx \sigma_{1}$ we have that $p, \gamma \vdash \sigma \approx \sigma_{1}^{\prime \prime}\left(x_{1}\right.$ and $x$ are not defined in $\gamma$ ). Since (4).a, and (3). $\alpha$, we can apply the inductive hypothesis to $e$, and derive that $\llbracket e \rrbracket, \sigma_{1}^{\prime \prime} \mathbb{\llbracket p} \rrbracket_{p} \iota, \sigma_{1}^{\prime \prime \prime}$ where $p, \gamma_{0} \vdash \sigma^{\prime \prime} \approx \sigma_{1}^{\prime \prime \prime}$. Therefore, $e_{2}, \sigma_{1}^{\prime \prime}$ đp $\quad$ false, $\sigma_{2}$, where $\sigma_{2}=\sigma_{1}^{\prime \prime \prime}[x \mapsto \iota]$, and $\left\{e_{3} ; e_{4}\right\}$ is evaluated.

From $p, \gamma_{0} \vdash \sigma^{\prime \prime} \approx \sigma_{1}^{\prime \prime \prime}$, as before, we can derive that also $p, \gamma_{0} \vdash \sigma^{\prime \prime} \approx \sigma_{2}$ ( $x$ is not defined in $\gamma_{0}$ ). Since (4).b, and (3). $\beta$, we can apply the inductive hypothesis to $e^{\prime}$, and derive that $\llbracket e^{\prime} \rrbracket, \sigma_{2} \widetilde{\prod}_{p} \rrbracket, \sigma_{2}^{\prime}$ where $p, \gamma^{\prime} \vdash \sigma^{\prime \prime \prime} \approx \sigma_{2}^{\prime}$ and from Lemma 15, $\sigma_{2}^{\prime}\left(x_{1}\right)=$

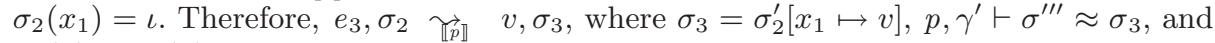
$\sigma_{3}(x)=\sigma_{2}(x)=\iota$.

Now we have to evaluate $\llbracket e_{4} \rrbracket$ in the store $\sigma_{3}$. From the rule for method call $($ meth $)$ : we evaluate first $\left(\phi_{1} @_{p} c\right)\left(x\right.$.imp) in the store $\sigma_{3}$. Since $p, \gamma^{\prime} \vdash \sigma^{\prime \prime \prime} \approx \sigma_{2}^{\prime}, \sigma_{2}^{\prime \prime}(\iota)=\sigma_{3}(\iota)$, and

$$
-\sigma_{3}(x)=\iota
$$




$$
\begin{aligned}
& -\sigma_{3}(\iota)=\left[\left[\text { imp }: \iota^{\prime} \cdots\right]\right]^{\text {Identity }}, \text { and } \\
& -\sigma_{3}\left(\iota^{\prime}\right)=[[\text { id }: \iota \cdots]]^{c^{\prime}} .
\end{aligned}
$$

Moreover, from (4).a, (3). $\alpha$ and Theorem 1 we have that $c^{\prime} \leq c$. Again from (4).b, (3). $\beta$ and Theorem 1 (in particular $p, \phi \vdash \sigma^{\prime \prime} \triangleleft \sigma^{\prime \prime \prime}$ ) we have that $\phi_{1} @_{p} c^{\prime} \leq \phi_{1} @_{p} c$. Therefore, the cast succeeds and we have that:

$$
\left(\phi_{1} @_{p} c\right)(x . \mathrm{imp}), \sigma_{3} \mathbb{\llbracket p} \rrbracket \quad \iota^{\prime}, \sigma_{3} \text { and } x_{1}, \sigma_{3} \quad \mathbb{\llbracket p} \rrbracket \quad v^{\prime}, \sigma_{3} .
$$

From $(3) \cdot \chi, \mathcal{M D}\left(p, c^{\prime}, m\right)=t m\left(t_{1} y_{1}\right) \phi$ block. Since the program is well formed from Figure 4, we have that

$$
(*) \quad p, \gamma^{\prime \prime} \vdash \text { block : } t^{\prime} \llbracket \gamma^{\prime \prime \prime} \llbracket \phi^{\prime \prime}
$$

where $\gamma^{\prime \prime}=t_{1} y_{1}, c^{\prime}$ this, $t^{\prime} \leq t$, and $\phi^{\prime \prime} \subseteq \phi^{\prime}$. From the definition of the translation of methods

$$
\begin{aligned}
& \llbracket t m\left(t_{1} y_{1} \phi \text { block } \rrbracket_{\text {meth }}(p, c) \triangleq\right. \\
& \text { the Type }(t) m\left(\llbracket t_{1} y_{1} \rrbracket_{v a r}\right)\{\} \llbracket b l o c k \rrbracket_{\text {expr }}\left(p, \gamma^{\prime \prime}\right)
\end{aligned}
$$

Consider the store $\sigma_{4}=\sigma_{3}\left[\right.$ this $\mapsto \iota^{\prime}, y_{1} \mapsto v^{\prime}$. It is immediate to see that $p, \gamma^{\prime \prime} \vdash$ $\bar{\sigma} \approx \sigma_{4}$. Applying the inductive hypothesis to $(3) \cdot \beta^{\prime},(*)$, we get:

$$
\llbracket b l o c k \rrbracket_{\operatorname{expr}}\left(p, \gamma^{\prime \prime}\right), \sigma_{4} \underset{\llbracket p \rrbracket}{ } v, \sigma_{5}
$$

where $p, \gamma^{\prime \prime \prime} \vdash \bar{\sigma}^{\prime} \approx \sigma_{5}$ and, since $\gamma^{\prime \prime}(x)=\gamma^{\prime \prime}\left(x_{1}\right)=\mathcal{U} d f$, from Lemma 15 and definition of $\sigma_{4}$ we have that $\sigma_{4}(x)=\sigma_{3}(x)=\sigma_{5}(x)$ and $\sigma_{4}\left(x_{1}\right)=\sigma_{3}\left(x_{1}\right)=\sigma_{5}\left(x_{1}\right)$. Let $\sigma_{6}=\sigma_{5}\left[\right.$ this $\mapsto \sigma_{3}$ (this), $\left.y_{1} \mapsto \sigma_{3}\left(y_{1}\right)\right]$ we have that

$$
\llbracket e_{4} \rrbracket, \sigma_{3} \quad \dddot{\llbracket p} \rrbracket \quad v, \sigma_{6}
$$

Let $\sigma^{\prime}$ be defined in (3). $\chi^{\prime}$. Since $p, \gamma^{\prime} \vdash \sigma^{\prime \prime \prime} \approx \sigma_{3}$ we have that $p, \gamma^{\prime} \vdash \sigma^{\prime} \approx \sigma_{6}$. Let $\sigma_{1}^{\prime}=\sigma_{6}\left[x \mapsto \sigma_{1}(x), x_{1} \mapsto \sigma_{1}\left(x_{1}\right)\right]$, it is immediate to show that $p, \gamma^{\prime} \vdash \sigma^{\prime} \approx \sigma_{1}^{\prime}$. This concludes the proof.

Consider rule (cast):

$$
\begin{aligned}
& e, \sigma \underset{p}{\longrightarrow} \iota, \sigma^{\prime} \\
& \sigma^{\prime}(\iota)=[[\ldots]]^{c^{\prime}} \\
& \frac{p \vdash c^{\prime} \leq c}{(c) e, \sigma \overleftrightarrow{p^{\prime}} \iota, \sigma^{\prime}}
\end{aligned}
$$

Since the expression is well-typed we have that

$$
\begin{aligned}
& a . p, \gamma \vdash e: c^{\prime} \llbracket \gamma^{\prime} \llbracket \phi \\
& \frac{b .\left(p \vdash c^{\prime} \leq c \text { or } p \vdash c \leq c^{\prime}\right)}{p, \gamma \vdash(c) e: c \llbracket \gamma^{\prime} \rrbracket \phi}
\end{aligned}
$$

Let $\llbracket(c) e \rrbracket_{\operatorname{expr}}(p, \gamma) \triangleq\left\{\right.$ Identity $x$; if $e_{1}$ then $e_{2}$ else null $\}$, where

$-e_{1}$ is $\left.\left(x=\llbracket e \rrbracket_{\text {expr }}(p, \gamma)\right) \neq \operatorname{null}\right)$, and

$-e_{2}$ is (theName(c)x.imp).id 
Let $\sigma_{1}^{\prime \prime}=\sigma_{1}[x \mapsto \operatorname{null}]$. From $p, \gamma \vdash \sigma \approx \sigma_{1}$, we have $p, \gamma \vdash \sigma \approx \sigma_{1}^{\prime \prime}$. From (5).a and $e, \sigma \underset{p}{\longrightarrow} \iota, \sigma^{\prime}$ we can apply the inductive hypothesis to $e$, and derive that $\llbracket e \rrbracket, \sigma_{1}^{\prime \prime} \widetilde{\llbracket p \rrbracket} \iota, \sigma_{1}^{\prime \prime \prime}$ and $p, \gamma^{\prime} \vdash \sigma^{\prime} \approx \sigma_{1}^{\prime \prime \prime}$. So $\sigma_{1}^{\prime \prime \prime}(\iota)=\left[\left[\text { imp : } \iota^{\prime}\right]\right]^{\text {Id }}$, and $\sigma_{1}^{\prime \prime \prime}\left(\iota^{\prime}\right)=[[\ldots, \text { id }: \iota]]^{\text {theName }\left(c^{\prime}\right)}$. Therefore, $\llbracket e_{1} \rrbracket, \sigma_{1}^{\prime \prime} \mathbb{\prod p}_{\mathbb{p}}$ true, $\sigma_{2}$, where $\sigma_{2}=\sigma_{1}^{\prime \prime \prime}[x \mapsto \iota]$, and the expression $e_{2}$ is evaluated.

Since $\sigma_{2}(x)($ imp $)=\iota^{\prime}, \sigma_{2}\left(\iota^{\prime}\right)($ id $)=\iota,(5)$.b, and Lemma 13, so $p \vdash$ theName $\left(c^{\prime}\right) \leq$

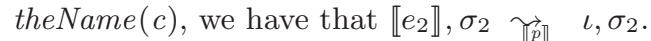

Let $\sigma_{1}^{\prime}=\sigma_{2}\left[x \mapsto \sigma_{1}(x)\right]$, we have that $\llbracket(c) e \rrbracket, \sigma_{1} \mathbb{\prod p} \rrbracket_{p} \iota, \sigma_{1}^{\prime}$, and from $p, \gamma^{\prime} \vdash \sigma^{\prime} \approx \sigma_{1}^{\prime \prime \prime}$, we get that $p, \gamma^{\prime} \vdash \sigma^{\prime} \approx \sigma_{1}^{\prime}$.

Consider rule ( $n$-cast), that is assume that $e, \sigma \underset{p}{ }$ null, $\sigma^{\prime}$. So from rule $(n$-cast), $(c) e, \sigma \underset{p}{\sim}$ null, $\sigma^{\prime}$. Consider $\llbracket(c) e \rrbracket$ as for rule (cast). We can apply the inductive hypothesis to $e$, and derive that $\llbracket e \rrbracket, \sigma_{1}^{\prime \prime} \prod_{\vec{p} \rrbracket}$ null, $\sigma_{1}^{\prime \prime \prime}$ and $p, \gamma^{\prime} \vdash \sigma^{\prime} \approx \sigma_{1}^{\prime \prime \prime}$. Therefore, $\llbracket e_{1} \rrbracket, \sigma_{1}^{\prime \prime} \widetilde{\prod p} \rrbracket$ false, $\sigma_{2}$, where $\sigma_{2}=\sigma_{1}^{\prime \prime \prime}\left[x \mapsto\right.$ null], and the expression $e_{2}$ is

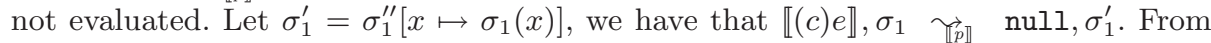
$p, \gamma^{\prime} \vdash \sigma^{\prime} \approx \sigma_{1}^{\prime \prime \prime}$, we get that $p, \gamma^{\prime} \vdash \sigma^{\prime} \approx \sigma_{1}^{\prime}$.

Let us now assume that the result of the evaluation is an exception. We will consider nullPntrExc raised during the evaluation of a field update and castExc raised by a cast expression.

Consider rule (e-null), and assume that the expression is an assignment to a field:

$$
\frac{e, \sigma \underset{p}{\sim} \operatorname{null}, \sigma^{\prime}}{e \cdot f=e^{\prime}, \sigma \underset{p}{\longrightarrow} \text { nullPntrExc, } \sigma^{\prime}}
$$

Consider the translation of the expression as for the case of rule $(a$-field $)$. Let $\sigma_{1}^{\prime \prime}=$ $\sigma_{1}\left[x_{1} \mapsto \operatorname{null}, x_{2} \mapsto v^{\prime}\right]$, where $v^{\prime}$ is an initial value of type theType $(t)$. From $p, \gamma \vdash \sigma \approx$ $\sigma_{1}$ we have that $p, \gamma \vdash \sigma \approx \sigma_{1}^{\prime \prime}\left(x_{1}\right.$ and $x_{2}$ are not defined in $\left.\gamma\right)$. Since the expression is well typed $p, \gamma \vdash e: c \rrbracket \gamma_{0} \rrbracket \phi_{0}$ for some $c, \gamma_{0}$, and $\phi_{0}$. So, from $e, \sigma \underset{p}{\vec{n}} \operatorname{null}, \sigma^{\prime}$, we can apply the inductive hypothesis to $e$, and derive that $\llbracket e \rrbracket, \sigma_{1}^{\prime \prime} \mathbb{\| p}_{\mathbb{p}}$ null, $\sigma_{1}^{\prime \prime \prime}$ where $p, \gamma_{0} \vdash \sigma^{\prime} \approx \sigma_{1}^{\prime \prime \prime}$. Therefore, $e_{1}, \sigma_{1}^{\prime \prime} \mathbb{\prod}_{p} \rrbracket$ false, $\sigma_{2}$, where $\sigma_{2}=\sigma_{1}^{\prime \prime \prime}\left[x_{1} \mapsto\right.$ null] , and $e_{2}$ is not evaluated.

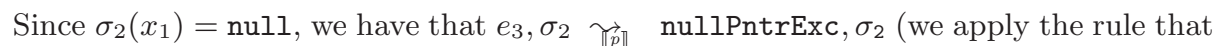
generate the exception to $x_{1}$.imp and then the rules for propagation of exceptions of Fig. 17).

Let $\sigma_{1}^{\prime}=\sigma_{2}\left[x_{1} \mapsto \sigma_{1}\left(x_{1}\right), x_{2} \mapsto \sigma_{1}\left(x_{2}\right)\right]$, then $\llbracket e . f=e^{\prime} \rrbracket, \sigma_{1} \underset{\mathbb{} p \rrbracket}{ }$ nullPntrExc, $\sigma_{1}^{\prime}$. Note that in this case it is important in the translation to avoid the evaluation of $e_{2}$, since it could raise a different exception or not terminate.

Consider rule (e-cast):

$$
\begin{aligned}
& e, \sigma \underset{p}{\longrightarrow} \iota, \sigma^{\prime} \\
& \sigma^{\prime}(\iota)=[[\ldots]]^{c^{\prime}} \\
& \frac{p \not c^{\prime} \leq c}{(c) e, \sigma \underset{p}{\longrightarrow} \operatorname{castExc}, \sigma^{\prime}}
\end{aligned}
$$

Let $\llbracket(c) e \rrbracket_{\text {expr }}(p, \gamma)$, and $\sigma_{1}^{\prime \prime}=\sigma_{1}[x \mapsto$ null $]$ be as for the case of rule (cast). From the inductive hypothesis applied to $e$, $\llbracket e \rrbracket, \sigma_{1}^{\prime \prime} \underset{\mathbb{u} \rrbracket}{\overleftrightarrow{p}} \iota, \sigma_{1}^{\prime \prime \prime}$ and $p, \gamma^{\prime} \vdash \sigma^{\prime} \approx \sigma_{1}^{\prime \prime \prime}$. So $\sigma_{1}^{\prime \prime \prime}(\iota)=\left[\left[\text { imp : } \iota^{\prime}\right]\right]^{\mathrm{Id}}$, and $\sigma_{1}^{\prime \prime \prime}\left(\iota^{\prime}\right)=$ 
$[[\ldots]]^{\text {theName }\left(c^{\prime}\right)}$. Therefore, $\llbracket e_{1} \rrbracket, \sigma_{1}^{\prime \prime} \underset{\llbracket p \rrbracket}{ }$ true, $\sigma_{2}$, where $\sigma_{2}=\sigma_{1}^{\prime \prime \prime}[x \mapsto \iota]$, and the expression $e_{2}$ is evaluated.

Since $\sigma_{2}(x)(\mathrm{imp})=\iota^{\prime}, \sigma_{2}\left(\iota^{\prime}\right)=[[\ldots]]^{\text {theName }\left(c^{\prime}\right)}$, and $p \not \forall c^{\prime} \leq c$, so from Lemma 13 also $p \nvdash$ theName $\left(c^{\prime}\right) \leq$ theName $(c)$ we have that

$\llbracket(c) x . i m p \rrbracket, \sigma_{2} \underset{\llbracket p \rrbracket}{ }$ castExc, $\sigma_{2}$. Applying the rules for propagation of exceptions we get that $\llbracket e_{2} \rrbracket, \sigma_{2} \widetilde{\pi p} \rrbracket$ castExc, $\sigma_{2}$.

Let $\sigma_{1}^{\prime}=\sigma_{2}\left[x \mapsto \sigma_{1}(x)\right]$, we have that $\llbracket(c) e \rrbracket, \sigma_{1} \underset{\mathbb{\|} p \rrbracket}{\|}$ castExc, $\sigma_{1}^{\prime}$. Note that the cast in the translation is essential to raise the exception, that otherwise would not occur since every Identity object has the field imp and every FickleObject has the field id.

Proof of: $\llbracket e \rrbracket, \sigma_{1} \underset{p}{\longrightarrow} w, \sigma_{1}^{\prime} \quad$ implies $\quad e, \sigma \underset{\mathbb{1} p}{\longrightarrow} \boldsymbol{w}, \sigma^{\prime}$ The result is proved by induction on the depth of the derivation tree of $\llbracket e \rrbracket, \sigma_{1} \overleftrightarrow{\llbracket p \rrbracket} w, \sigma_{1}^{\prime}$. Note that, since $e$ is well-typed, from Theorem 2 we have that also $\llbracket e \rrbracket$ is well-typed so from Theorem 1 its evaluation cannot produce stuckErr and $w$ is either a value or $w \in\{$ castExc, nullPntrExc $\}$. We will consider the translation of the following expressions: identifiers, object creation, re-classification, field update, and cast. The other cases are similar.

Consider $\llbracket i \mathrm{~d} \rrbracket \triangleq$ id. Since $w \neq$ stuckErr the only rule applicable is $\widehat{\llbracket i d \rrbracket, \sigma_{1} \underset{\mathbb{p} \rrbracket}{ }} \sigma_{1}(\mathrm{id}), \sigma_{1}$. From $p, \gamma \vdash \sigma \approx \sigma_{1}$ we have that $\sigma(\mathrm{id})=\sigma_{1}(\mathrm{id})$. Therefore also id, $\sigma \underset{p}{\longrightarrow} \sigma($ id),$\sigma$.

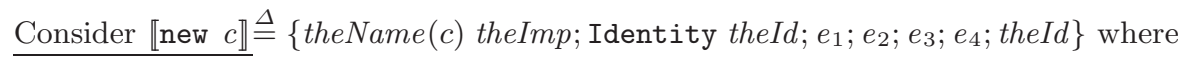

$-e_{1}$ is theId $=$ new Identity,

$-e_{2}$ is theImp = new theName $(c)$,

$-e_{3}$ is theImp.id $=$ theId, and

$-e_{4}$ is theId.imp $=$ theImp.

and $\gamma($ theId $)=\gamma($ theImp $)=U d f$. Assume that $c \neq$ Object, so theName $(c)=c$. (The case $c=$ Object is simpler since $\mathcal{F} s(p$, Object $)=\emptyset$.) From the definition of the operational semantics, and the fact that: $c \leq$ FickleObject has the field id and an object of class Identity has the field imp (so the evaluation of the expressions cannot produce an exception), as for the case of the corresponding "only if" proof, we have that:

$$
\llbracket \text { new } c \rrbracket \rrbracket, \sigma_{1} \quad \widetilde{p} \rrbracket \quad \iota, \sigma_{1}^{\prime}
$$

where

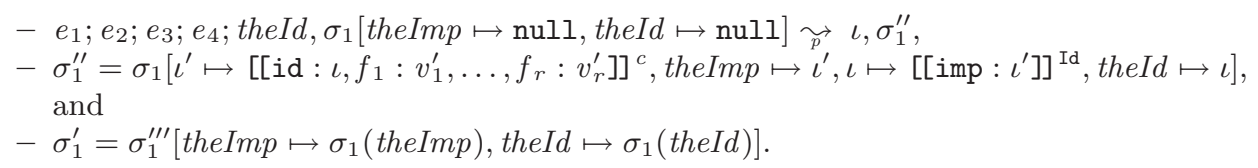

We choose $\iota$ and $\iota^{\prime}$ such that they are new both in $\sigma_{1}$ and $\sigma$. Since $\iota$ is new in $\sigma$ we have

$$
\text { new } c, \sigma \underset{p}{\longrightarrow} \iota, \sigma^{\prime}
$$

where $\sigma^{\prime}=\sigma\left[\iota \mapsto\left[\left[f_{1}: v_{1}, \ldots, f_{r}: v_{r}\right]\right]^{c}\right]$, and $v_{l}$ is initial for $\mathcal{F}\left(p, c, f_{l}\right), 1 \leq l \leq r$. As for the case of the corresponding "only if" proof, using Lemma 14 we can show that $p, \gamma^{\prime} \vdash \sigma^{\prime} \approx \sigma_{1}^{\prime}$. 


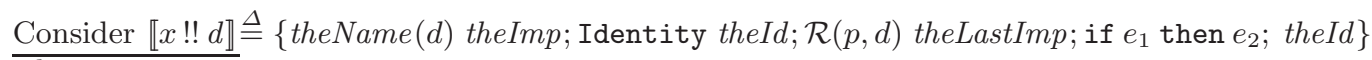
where

$$
\begin{aligned}
& -e_{1} \text { is }((\text { theId }=x) \neq \text { null }) \text {, } \\
& -e_{2} \text { is } \\
& \{\text { theImp }=\text { new theName }(d) ; \text { theLastImp }=(\mathcal{R}(p, d)) \text { theId.imp; } \\
& \text { theImp.id = theId; theId.imp = theImp; } \\
& e_{1}^{\prime} ; \ldots e_{r}^{\prime} \\
& \} \\
& -e_{i}^{\prime} \text { is theImp. } f_{i}=\text { theLastImp. } f_{i} \text { for } 1 \leq i \leq r \text {, and } \\
& \gamma(\text { theId })=\gamma(\text { theLastImp })=\gamma(\text { theImp })=U d f . \\
& \text { If } \sigma_{1}(x)=\text { null, then } \llbracket x ! ! d \rrbracket, \sigma_{1} \quad \mathbb{\llbracket p} \rrbracket \quad \text { null, } \sigma_{1} \text { since } \\
& e_{1}, \sigma_{1}\left[\text { theImp, theId, theLastImp } \mapsto \text { null] } \widetilde{\llbracket p} \rrbracket \text { false, } \sigma_{1}[\text { theImp, theId, theLastImp } \mapsto \text { null] }\right.
\end{aligned}
$$

Since the expression is well-typed $d \neq$ Object, and theName $(d)=d$. Applying rule $(n-r e c l)$ also $x ! ! d, \sigma$ đֶp $\rrbracket$ null, $\sigma$ and the result holds.

Consider now the case $\sigma_{1}(x) \neq$ null. From $p, \gamma^{\prime} \vdash \sigma \approx \sigma_{1}$ we have $\sigma(x) \neq$ null. Moreover, since the expression is well-typed $\gamma(x)=c$, so from $p, \gamma \vdash \sigma \diamond, \sigma(x)=\iota$ and $\sigma(\iota)=[[\cdots]]^{c^{\prime}}$ for some $c^{\prime} \leq c$. Again from $p, \gamma^{\prime} \vdash \sigma \approx \sigma_{1}$, we derive that

$$
\sigma_{1}(x)=\iota \quad \sigma_{1}(\iota)=\left[\left[\text { imp }: \iota^{\prime}\right]\right]^{\text {Id }} \quad \sigma_{1}\left(\iota^{\prime}\right)=[[\text { id }: \iota, \cdots]]^{c^{\prime}}
$$

From the definition of the operational semantics of blocks, and the fact that null is the initial value for an object, we have that:

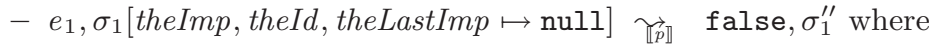
$\sigma_{1}^{\prime \prime}=\sigma_{1}[$ theImp, theLastImp $\mapsto$ null, theId $\mapsto \iota]$,

- theImp = new $d, \sigma_{1}^{\prime \prime} \widetilde{\pi} \rrbracket \iota^{\prime \prime}, \sigma_{2}$ where $\iota^{\prime \prime}$ is new and $\sigma_{2}=\sigma_{1}^{\prime \prime}\left[\right.$ theImp $\mapsto \iota^{\prime \prime}, \iota^{\prime \prime} \mapsto\left[\left[\right.\right.$ id $: \operatorname{null}, f_{1}: v_{1}^{\prime \prime}, \ldots, f_{r}: v_{r}^{\prime \prime}, f_{r+1}: v_{r+1}^{\prime \prime}, \ldots, f_{r+q}:$ $\left.\left.v_{r+q}^{\prime \prime}\right]\right]^{d}$, theImp $\left.\mapsto \iota^{\prime \prime \prime}\right]$ where $v_{l}^{\prime \prime}$ is initial for the Type $\left(\mathcal{F}\left(p, d, f_{l}\right)\right), l \in\{1, \ldots, r+q\}$

- from equation (6), the fact that the expression is well typed, therefore $\mathcal{R}(p, d)=$ $\mathcal{R}\left(p, c^{\prime}\right)$, we have that

$$
\text { theLastImp }=(\mathcal{R}(p, d)) \text { theId.imp, } \sigma_{2} \underset{\mathbb{p} \rrbracket}{ } \iota^{\prime}, \sigma_{3}
$$

where $\sigma_{3}=\sigma_{2}\left[\right.$ theLastImp $\left.\mapsto \iota^{\prime}\right]$ (neither the cast operation, nor the access to field imp produce an exception)

- theImp.id = theId; theId.imp $=$ theImp; $\sigma_{3} \underset{\mathbb{} p}{ } \rrbracket \quad \iota^{\prime \prime}, \sigma_{4}$ where $\sigma_{4}=\sigma_{3}[\iota \mapsto[$ [imp : $\left.\left.\iota^{\prime \prime}\right]\right]^{\mathrm{Id}}, \iota^{\prime \prime} \mapsto\left[\left[\mathrm{id}: \iota^{\prime \prime}, f_{1}: v_{1}^{\prime \prime}, \ldots, f_{r}: v_{r}^{\prime \prime}, f_{r+1}: v_{r+1}^{\prime \prime}, \ldots, f_{r+q}: v_{r+q}^{\prime \prime}\right]^{d}\right.$, the Imp $\mapsto$ $\left.\iota^{\prime \prime \prime}\right]$, and finally

$-e_{1}^{\prime} ; \ldots e_{r}^{\prime}, \sigma_{4} \mathbb{\prod}_{p} \rrbracket \quad \iota^{\prime \prime}, \sigma_{1}^{\prime \prime}$ where $\sigma_{1}^{\prime \prime}=\sigma_{4}\left[\iota^{\prime \prime} \mapsto\left[\right.\right.$ [id : $\iota^{\prime \prime}, f_{1}: v_{1}, \ldots, f_{r}: v_{r}, f_{r+1}:$ $\left.\left.v_{r+1}^{\prime \prime}, \ldots, f_{r+q}: v_{r+q}^{\prime \prime}\right]^{d}\right]$ where $\left\{f_{1}, \ldots, f_{r}\right\}$ are the fields of the class $\mathcal{R}(p, d)$ and $v_{i}=\sigma_{1}(x)\left(f_{i}\right)$ for $1 \leq i \leq r$.

Let $\sigma_{1}^{\prime}=\sigma_{1}^{\prime \prime}\left[\right.$ theImp $\mapsto \sigma_{1}($ theImp $)$, theId $\mapsto \sigma_{1}$ (theId), theLastThis $\mapsto \sigma_{1}$ (theLastThis)]. We derive that

$$
\llbracket x ! ! d \rrbracket, \sigma_{1} \underset{\llbracket p \rrbracket}{\llbracket} \quad \iota, \sigma_{1}^{\prime} .
$$


On the other hand, since $\sigma(x)=\iota, \sigma(\iota)=[[\cdots]]^{c^{\prime}}$, and $\left\{f_{1}, \ldots, f_{r}\right\}$ are the fields of the class $\mathcal{R}(p, d)$ from rule (recl) we have that

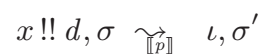

where $\sigma^{\prime}=\sigma\left[\iota \mapsto\left[\left[f_{1}: v_{1}^{\prime}, \ldots, f_{r}: v_{r}^{\prime}, f_{r+1}: v_{r+1}^{\prime}, \ldots, f_{r+q}: v_{r+q}^{\prime}\right]\right]^{d}, v_{i}^{\prime}=\sigma(x)\left(f_{i}\right)\right.$ for $1 \leq i \leq r$, and $v_{i}^{\prime}$ is the initial value for $\mathcal{F}\left(p, d, f_{i}\right), r \leq i \leq r+q$.

To show that $p, \gamma^{\prime} \vdash \sigma^{\prime} \approx \sigma_{1}^{\prime}$ we have to prove that the three clauses of Definition 2 are satisfied for $\sigma^{\prime}$ and $\sigma_{1}^{\prime}$. From the definition of $\sigma^{\prime}$ and $\sigma_{1}^{\prime}$ and the fact that $p, \gamma \vdash$ $\sigma \approx \sigma_{1}$ we have that for all $x^{\prime}, \sigma^{\prime}\left(x^{\prime}\right)=\sigma_{1}^{\prime}\left(x^{\prime}\right)$. Therefore, clause 1 . of Definition 2 holds, and since the only field id that is updated in $\sigma_{1}^{\prime}$ is the one of a newly created location also $\sigma_{1}^{\prime}$ (this)(id) $=\sigma_{1}$ (this)(id) and clause 2 . holds. To conclude the proof, we have to verify clause 3 . for the address $\iota$. Let

$$
\begin{aligned}
& -\sigma^{\prime}(\iota)=\left[\left[f_{1}: v_{1}^{\prime}, \ldots, f_{r}: v_{r}^{\prime}\right]\right]^{c}, \sigma_{1}^{\prime}(\iota)=\left[\left[\text { imp }: \iota^{\prime \prime \prime}\right]\right]^{\mathrm{Id}}, \\
& -\sigma_{1}^{\prime}\left(\iota^{\prime \prime \prime}\right)=\left[\left[\text { id }: \iota, f_{1}: v_{1}, \ldots, f_{r}: v_{r}, f_{r+1}: v_{r+1}^{\prime \prime}, \ldots, f_{r+q}: v_{r+q}^{\prime \prime}\right]\right]^{d}
\end{aligned}
$$

From $p, \gamma \vdash \sigma \approx \sigma_{1}$ for all $i, 1 \leq i \leq r, v_{i}=v_{i}^{\prime}$. Moreover, for all $i, r \leq i \leq r+q, v_{i}^{\prime}$ is an initial value for $\mathcal{F}\left(p, d, f_{r+i}\right)$ and $v_{r+i}^{\prime \prime}$ is an initial value for the Type $\left(\mathcal{F}\left(p, d, f_{r+i}\right)\right)$. Therefore, from Lemma $14, v_{r+i}=v_{r+i}^{\prime \prime}$, and clause 3 . holds. This concludes the proof that $p, \gamma^{\prime} \vdash \sigma^{\prime} \approx \sigma_{1}^{\prime}$.

The case in which the expression is 【this !! $d \rrbracket$ is similar.

$\underline{\text { Consider } \llbracket e . f \rrbracket}=\left\{\right.$ Identity $x_{1}$; theType $(c) x_{2}$; if $e_{1}$ then $e_{2}$ else $\left.\left\{e_{3} ; e_{4}\right\}\right\}$ where

$-e_{1}$ is $\operatorname{isnull}\left(x_{1}=\llbracket e \rrbracket_{\text {expr }}(p, \gamma)\right.$

$-e_{2}$ is null. $f=x_{2}$

$-e_{3}$ is $x_{2}=\llbracket e^{\prime} \rrbracket_{\operatorname{expr}}\left(p, \gamma_{0}\right)$

$-e_{4}$ is $\left(\left(\phi^{\prime} @_{p} c\right)\left(x_{1} \cdot \mathrm{imp}\right)\right) . f=x_{2}$

and $\gamma\left(x_{1}\right)=\gamma\left(x_{2}\right)=\mathcal{U} d f$. From the fact that the expression is well-typed, we have

$$
\begin{aligned}
& \text { a. } p, \gamma \vdash e: c \rrbracket \gamma_{0} \llbracket \phi_{0} \\
& \text { b. } p, \gamma_{0} \vdash e^{\prime}: t \rrbracket \gamma^{\prime} \llbracket \phi^{\prime} \\
& c . \mathcal{F}\left(p, \phi^{\prime} @_{p} c, f\right)=t^{\prime} \quad p \vdash t \leq t^{\prime} \quad \phi=\phi \cup \phi^{\prime} \\
& \hline p, \gamma \vdash e \cdot f=e^{\prime}: t \rrbracket \gamma^{\prime} \rrbracket \phi
\end{aligned}
$$

Note that, since $\mathcal{F} s(p$, Object $)=\emptyset, c \neq$ Object, and therefore theName $(c)=c$. Let $\sigma_{1}^{\prime \prime}=\sigma_{1}\left[x_{1} \mapsto \operatorname{null}, x_{2} \mapsto v^{\prime}\right]$, where $v^{\prime}$ is an initial value of type the Type $(t)$. Since $p, \gamma \vdash$ $\sigma \approx \sigma_{1}$ and $x_{1}$ and $x_{2}$ are not defined in $\gamma$ also $p, \gamma \vdash \sigma \approx \sigma_{1}^{\prime \prime}$. From $\llbracket e . f=e^{\prime} \rrbracket, \sigma_{1} \leadsto$ $\llbracket p \rrbracket \quad w, \sigma_{1}^{\prime}$ we derive that:

$$
\text { if } e_{1} \text { then } e_{2} \text { else }\left\{e_{3} ; e_{4}\right\}, \sigma_{1}^{\prime \prime} \dddot{\dddot{p}} \rrbracket w, \sigma_{1}^{\prime \prime \prime}
$$

where $\sigma_{1}^{\prime}=\sigma_{1}^{\prime \prime \prime}\left[x_{1} \mapsto \sigma_{1}\left(x_{1}\right), x_{2} \mapsto \sigma_{1}\left(x_{2}\right)\right]$. Therefore,

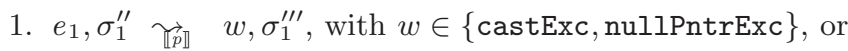

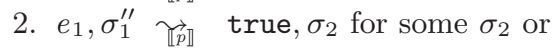

3. $e_{1}, \sigma_{1}^{\prime \prime} \underset{\llbracket p 》}{\mathbb{p} \rrbracket}$ false, $\sigma_{2}$ for some $\sigma_{2}$. 
1. For the first case observe that, using the propagation rules for exceptions

$$
\llbracket e . f=e^{\prime} \rrbracket, \sigma_{1} \quad \dddot{p} \rrbracket \quad w, \sigma_{1}^{\prime}
$$

Moreover, since an assignment does not produce an exception it must be the case that

$$
\llbracket e \rrbracket, \sigma_{1}^{\prime \prime} \widetilde{\llbracket p} \rrbracket, \sigma_{1}^{\prime \prime \prime}
$$

Since $e$ is well-typed, then $p, \gamma \vdash e: c \rrbracket \gamma_{0} \rrbracket \phi_{0}$ for some $c, \gamma_{0}$, and $\phi_{0}$. So, from $p, \gamma \vdash \sigma \approx \sigma_{1}^{\prime \prime}$ we can apply the inductive hypothesis to $e$ and derive that: $e, \sigma \widetilde{p_{p} \rrbracket} w, \sigma^{\prime}$. From the propagation rules for exceptions we derive

$$
\text { e.f }=e^{\prime}, \sigma \underset{\mathbb{p} \rrbracket}{\mathbb{p} \rrbracket} \quad w, \sigma^{\prime}
$$

that proves the result.

2. Assume that the evaluation of $e_{1}$ produces false. Therefore

$$
\llbracket e \rrbracket, \sigma_{1}^{\prime \prime} \widetilde{\natural p} \rrbracket, \sigma_{2}^{\prime \prime}
$$

where $\sigma_{2}=\sigma_{2}^{\prime \prime}\left[x_{1} \mapsto v\right]$ and $v \neq$ null. From $p, \gamma \vdash \sigma \approx \sigma_{1}$ we have that $p, \gamma \vdash$ $\sigma \approx \sigma_{1}^{\prime \prime}\left(x_{1}\right.$ and $x_{2}$ are not defined in $\gamma$ ). From (7).a, and (9), we can apply the inductive hypothesis to $e$, and derive that $e, \sigma \underset{\mathbb{p} p}{ } \quad v, \sigma^{\prime \prime}$ where $p, \gamma_{0} \vdash \sigma^{\prime \prime} \approx \sigma_{2}^{\prime \prime}$ and since $x_{1}$ is not defined in $\gamma_{0}$ also $p, \gamma_{0} \vdash \sigma^{\prime \prime} \approx \sigma_{2}$. From (8) (since $e_{1}$ evaluates to false) we derive that

$-e_{3}, \sigma_{2} \mathbb{\llbracket p} \quad w, \sigma_{1}^{\prime \prime \prime}$, with $w \in\{$ castExc, nullPntrExc $\}$, or

$-e_{3}, \sigma_{2} \stackrel{v^{\prime}}{ } v^{\prime}, \sigma_{3}$ for some $\sigma_{3}$ and $v^{\prime}$.

In the first case, as for the corresponding case of the evaluation of $e_{1}$,

$$
\llbracket e . f=e^{\prime} \rrbracket, \sigma_{1} \quad \dddot{\Perp p}_{\mathbb{p}} \quad w, \sigma_{1}^{\prime} .
$$

From the inductive hypothesis on $e^{\prime}$ and the propagation rules for exceptions $e . f=$ $e^{\prime}, \sigma \quad \widetilde{\llbracket p} w, \sigma^{\prime}$.

In the second case (the evaluation of $e_{3}$ produces $v^{\prime}$ ) we have that

$$
\llbracket e^{\prime} \rrbracket, \sigma_{2} \quad \llbracket \vec{p} \rrbracket \quad v^{\prime}, \sigma_{3}^{\prime \prime}
$$

where $\sigma_{3}=\sigma_{3}^{\prime \prime}\left[x_{2} \mapsto v^{\prime}\right]$. (Note that since $\gamma_{0}\left(x_{1}\right)=\mathcal{U} d f$ we also have that $\sigma_{3}\left(x_{1}\right)=$ $\sigma_{2}\left(x_{1}\right)=\iota$.) From (7).b, (10), and $p, \gamma_{0} \vdash \sigma^{\prime \prime} \approx \sigma_{2}$ we can apply the inductive hypothesis to $e^{\prime}$ and derive that $e^{\prime}, \sigma^{\prime \prime} \overleftrightarrow{\llbracket p} v^{\prime}, \sigma^{\prime \prime \prime}$ where $p, \gamma^{\prime} \vdash \sigma^{\prime \prime \prime} \approx \sigma_{3}^{\prime \prime}$, and since $x_{2}$ is not defined in $\gamma^{\prime}$ then also $p, \gamma^{\prime} \vdash \sigma^{\prime \prime \prime} \approx \sigma_{3}$.

From (7).a, and Theorem 1, p, $\sigma^{\prime \prime} \vdash v \triangleleft c$, therefore $v=\iota$ and $\sigma^{\prime \prime}(\iota)=[[\cdots]]^{c^{\prime}}$ where $c^{\prime} \leq c$. From $p, \gamma_{0} \vdash \sigma^{\prime \prime} \approx \sigma_{2}$ also $\sigma_{2}(\iota)=\left[\left[\text { imp : } \iota^{\prime}\right]\right]^{\text {Id }}, \sigma_{2}\left(\iota^{\prime}\right)=[[$ id : $\iota \cdots]]^{c^{\prime}}$, and $\sigma_{2}\left(x_{1}\right)=\iota$. Moreover, (7).b, and Theorem 1, implies that $p, \phi^{\prime} \vdash$ $\sigma^{\prime \prime} \triangleleft \sigma^{\prime \prime \prime}$ and $p, \gamma^{\prime} \vdash \sigma^{\prime \prime \prime} \diamond$. So $\sigma^{\prime \prime \prime}(\iota)=[[\cdots]]^{c^{\prime \prime}}$, and $\phi^{\prime} @_{p} c^{\prime}=\phi^{\prime} @_{p} c^{\prime \prime}$. So (7).c, $\phi^{\prime} @_{p} c^{\prime} \leq \phi^{\prime} @_{p} c$ implies that field $f$ is defined for $\sigma^{\prime \prime \prime}(\iota)$. From $p, \gamma^{\prime} \vdash \sigma^{\prime \prime \prime} \approx \sigma_{3}$ we have that $\sigma_{3}(\iota)=\left[\left[\text { imp : } \iota^{\prime \prime}\right]\right]^{\text {Id }}, \sigma_{2}\left(\iota^{\prime \prime}\right)=[[\text { id }: \iota \cdots]]^{c^{\prime \prime}}$ (for some $\iota^{\prime \prime}$ ) and field $f$ is defined for $\sigma_{3}\left(\iota^{\prime}\right)$. Therefore,

$$
e_{4}, \sigma_{3} \quad \dddot{\imath} \rrbracket \quad v^{\prime}, \sigma_{3}\left[\iota^{\prime \prime} \mapsto \sigma_{3}\left(\iota^{\prime \prime}\left[f \mapsto v^{\prime}\right]\right]\right.
$$

and $\llbracket e . f=e^{\prime} \rrbracket, \sigma_{1} \quad \widetilde{\sim p} \rrbracket \quad v^{\prime}, \sigma_{1}^{\prime}$ where $\sigma_{1}^{\prime}=\sigma_{3}\left[x_{1} \mapsto \sigma_{1}\left(x_{1}\right), x_{2} \mapsto \sigma_{1}\left(x_{2}\right), \iota^{\prime \prime} \mapsto\right.$ $\sigma_{3}\left(\iota^{\prime \prime}\left[f \mapsto v^{\prime}\right]\right]$.

On the other hand, 
$-e, \sigma \widetilde{\mathbb{p} \rrbracket} \iota, \sigma^{\prime \prime}$

$-e^{\prime}, \sigma^{\prime \prime} \dddot{\prod} \rrbracket \quad v^{\prime}, \sigma^{\prime \prime \prime}$, and

- field $f$ defined for $\sigma^{\prime \prime \prime}(\iota)$,

implies that

$$
\text { e.f }=e^{\prime}, \sigma \underset{\llbracket p \rrbracket}{ } \iota, \sigma^{\prime}
$$

where $\sigma^{\prime}=\sigma^{\prime \prime \prime}\left[\iota^{\prime} \mapsto \sigma^{\prime}(\iota)\left[f \mapsto v^{\prime}\right]\right]$. From $p, \gamma^{\prime} \vdash \sigma^{\prime \prime \prime} \approx \sigma_{3}$ it is easy to show that $p, \gamma^{\prime} \vdash \sigma^{\prime} \approx \sigma_{1}^{\prime}$. This concludes the proof of the case in which the evaluation of $e_{1}$ was false.

3. Assume, now, that $\llbracket e_{1} \rrbracket, \sigma_{1}^{\prime \prime} \mathbb{\prod}_{\mathbb{p} \rrbracket}$ true, $\sigma_{2}$. Therefore

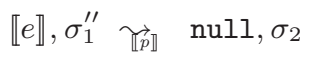

and $\sigma_{2}\left(x_{1}\right)=$ null. From $p, \gamma \vdash \sigma \approx \sigma_{1}$ we have that $p, \gamma \vdash \sigma \approx \sigma_{1}^{\prime \prime}\left(x_{1}\right.$ and $x_{2}$ are not defined in $\gamma$ ). From (7).a, and (11), we can apply the inductive hypothesis to $e$, and derive that $e, \sigma \widetilde{\pi p}$ null, $\sigma^{\prime \prime}$ where $p, \gamma_{0} \vdash \sigma^{\prime \prime} \approx \sigma_{2}$.

Therefore, null. $f, \sigma_{2} \underset{\llbracket p \rrbracket}{ }$ nullPntrExc, $\sigma_{2}$ and from the propagation rules

$$
\llbracket e . f=e^{\prime} \rrbracket, \sigma_{1} \quad \widetilde{\natural p} \rrbracket \quad \text { nullPntrExc }, \sigma_{1}^{\prime}
$$

where $\sigma_{1}^{\prime}=\sigma_{2}\left[x_{1} \mapsto \sigma_{1}\left(x_{1}\right), x_{2} \mapsto \sigma_{1}\left(x_{2}\right)\right]$.

On the other hand, applying rule (e-null),

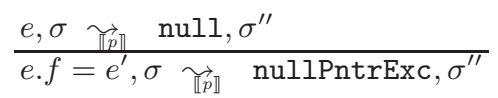

and $p, \gamma_{0} \vdash \sigma^{\prime \prime} \approx \sigma_{2}$ implies $p, \gamma_{0} \vdash \sigma^{\prime \prime} \approx \sigma_{1}^{\prime}$

The proof for e.m $\left(e^{\prime}\right)$ is similar.

$\underline{\text { Consider } \llbracket(c) e \rrbracket_{\text {expr }} \triangleq} \triangleq\left\{\right.$ theType $(t) x$; if $e_{1}$ then $e_{2}$ else null $\}$, where

$-e_{1}$ is $\left.\left(x=\llbracket e \rrbracket_{\text {expr }}(p, \gamma)\right) \neq \mathrm{null}\right)$, and

$-e_{2}$ is (theName(c)x.imp).id

Since the expression is well-typed

$$
\begin{aligned}
& a . p, \gamma \vdash e: c^{\prime} \llbracket \gamma^{\prime} \llbracket \phi \\
& \frac{b \cdot\left(p \vdash c^{\prime} \leq c \text { or } p \vdash c \leq c^{\prime}\right)}{p, \gamma \vdash(c) e: c \llbracket \gamma^{\prime} \rrbracket \phi}
\end{aligned}
$$

holds. From $\llbracket(c) e \rrbracket_{\text {expr }}, \sigma_{1} \underset{p}{\vec{p}} w, \sigma_{1}^{\prime}$ and the definition of the operational semantics of blocks we have that

$$
\text { if } e_{1} \text { then } e_{2} \text { else null, } \sigma_{1}^{\prime \prime} \underset{p}{\longrightarrow} w, \sigma_{1}^{\prime \prime \prime}
$$

where $\sigma_{1}^{\prime \prime}=\sigma_{1}[x \mapsto \mathrm{null}]$ and $\sigma_{1}^{\prime}=\sigma_{1}^{\prime \prime \prime}\left[x \mapsto \sigma_{1}(x)\right]$. Therefore,

1. $e_{1}, \sigma_{1}^{\prime \prime} \widetilde{\prod p} w, \sigma_{1}^{\prime \prime \prime}$, with $w \in\{$ castExc, nullPntrExc $\}$, or

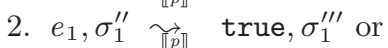

3. $e_{1}, \sigma_{1}^{\prime \prime} \underset{\mathbb{p}}{\mathbb{p} \rrbracket}$ false, $\sigma_{1}^{\prime \prime \prime}$. 
1. For the first case observe that it must be that,

$$
\llbracket e \rrbracket, \sigma_{1}^{\prime \prime} \mathbb{\imath}_{p} \rrbracket \quad w, \sigma_{1}^{\prime \prime \prime}
$$

Using (12).a, and $p, \gamma \vdash \sigma \approx \sigma_{1}^{\prime \prime}$ we can apply the inductive hypothesis to $e$ and derive that: $e, \sigma \stackrel{\mathbb{\|} p \rrbracket}{\sim} w, \sigma^{\prime}$ and $p, \gamma \vdash \sigma \approx \sigma^{\prime}$. From the propagation rules for exceptions we derive that

$$
\llbracket(c) e \rrbracket_{e x p r}, \sigma_{1} \underset{\llbracket p \rrbracket}{ } w, \sigma_{1}^{\prime}
$$

and

$$
(c) e, \sigma \widetilde{\llbracket p} \rrbracket \quad w, \sigma^{\prime}
$$

and $p, \gamma \vdash \sigma \approx \sigma^{\prime}$ implies $p, \gamma \vdash \sigma \approx \sigma^{\prime}$ which prove the result.

2. Assume that the evaluation of $e_{1}$ produces true. Therefore

$$
\llbracket e \rrbracket, \sigma_{1}^{\prime \prime} \dddot{\natural p} \rrbracket \quad v, \sigma_{2}
$$

where $\sigma_{1}^{\prime \prime \prime}=\sigma_{2}[x \mapsto v]$ and $v \neq$ null. From $p, \gamma \vdash \sigma \approx \sigma_{1}$ we have that $p, \gamma \vdash$ $\sigma \approx \sigma_{1}^{\prime \prime}$ ( $x$ is not defined in $\gamma$ ). From (12).a, and (14), we can apply the inductive hypothesis to $e$, and derive that $e, \sigma \underset{\mathbb{p} \rrbracket}{\|} v, \sigma^{\prime}$ where $p, \gamma^{\prime} \vdash \sigma^{\prime} \approx \sigma_{2}$ and since $x$ is not defined in $\gamma^{\prime}$ also $p, \gamma^{\prime} \vdash \sigma^{\prime} \approx \sigma_{1}^{\prime \prime \prime}$.

Observe that, (12).a, and Theorem 1, implies that $p, \sigma^{\prime} \vdash v \triangleleft c^{\prime}$, therefore $v=\iota$ and $\sigma^{\prime}(\iota)=[[\cdots]]^{c^{\prime \prime}}$ where $c^{\prime \prime} \leq c^{\prime}$. From $p, \gamma^{\prime} \vdash \sigma^{\prime} \approx \sigma_{1}^{\prime \prime \prime}$ also $\sigma_{1}^{\prime \prime \prime}(\iota)=\left[\left[\text { imp : } \iota^{\prime}\right]\right]^{\text {Id }}$, $\sigma_{1}^{\prime \prime \prime}\left(\iota^{\prime}\right)=[[\text { id }: \iota \cdots]]^{\text {theName }\left(c^{\prime \prime}\right)}$, and $\sigma_{1}^{\prime \prime \prime}(x)=\iota$. For the evaluation of $e_{2}$ there are two cases:

$-e_{2}, \sigma_{1}^{\prime \prime \prime} \mathbb{\prod} \rrbracket \quad$ castExc, $\sigma_{1}^{\prime \prime \prime}$ if $c^{\prime} \not \leq c$, or

$-e_{2}, \sigma_{1}^{\prime \prime \prime} \underset{\mathbb{p} \rrbracket}{\|} \iota, \sigma_{1}^{\prime \prime \prime}$ if $c^{\prime} \leq c$.

For the first case we have that from the propagation rules for exceptions

$$
\llbracket(c) e \rrbracket, \sigma_{1} \underset{\llbracket p \rrbracket}{ } \text { castExc, } \sigma_{1}^{\prime}
$$

and from the application of the rule $\left(e\right.$-cast) also $(c) e, \sigma \quad \widetilde{\prod p} \rrbracket$ castExc, $\sigma^{\prime}$.

For the second case

$$
\llbracket(c) e \rrbracket, \sigma_{1} \widetilde{\llbracket p} \rrbracket \quad \iota, \sigma_{1}^{\prime}
$$

On the other hand we can apply rule (cast) and get $(c) e, \sigma \underset{\mathbb{p} p \rrbracket}{ } \iota, \sigma^{\prime}$. From $p, \gamma^{\prime} \vdash \sigma^{\prime} \approx \sigma_{1}^{\prime \prime \prime}$ the definition of $\sigma_{1}^{\prime}$, and the fact that $\gamma(x)=\mathcal{U} d f$ we have $p, \gamma^{\prime} \vdash \sigma^{\prime} \approx \sigma_{1}^{\prime}$.

3. If $e_{1}, \sigma_{1}^{\prime \prime} \underset{\llbracket p \rrbracket}{\widetilde{f}}$ false, $\sigma_{1}^{\prime \prime \prime}$ then

$$
\llbracket e \rrbracket, \sigma_{1}^{\prime \prime} \underset{\llbracket p \rrbracket}{n} \operatorname{null}, \sigma_{1}^{\prime \prime \prime}
$$

Applying $(f$-cond $)$ we have

$$
\text { if } e_{1} \text { then } e_{2} \text { else null, } \sigma_{1}^{\prime \prime} \underset{p}{\longrightarrow} \operatorname{null}, \sigma_{1}^{\prime \prime \prime}
$$

and finally

$$
\llbracket(c) e \rrbracket, \sigma_{1} \underset{p}{\longrightarrow} \operatorname{null}, \sigma_{1}^{\prime}
$$

From (12).a, we can apply the inductive hypothesis to $e$, and derive that $e, \sigma \leadsto$ $\llbracket p \rrbracket$ null, $\sigma^{\prime}$ where $p, \gamma^{\prime} \vdash \sigma^{\prime} \approx \sigma_{1}^{\prime \prime \prime}$. Therefore, applying rule ( $n$-cast) we have

$$
(c) e, \sigma \quad \widetilde{p} \rrbracket \text { null, } \sigma^{\prime}
$$

From $p, \gamma^{\prime} \vdash \sigma^{\prime} \approx \sigma_{1}^{\prime \prime \prime}$ and the definition of $\sigma_{1}^{\prime}$ we derive that $p, \gamma^{\prime} \vdash \sigma^{\prime} \approx \sigma_{1}^{\prime}$. This concludes the proof. 